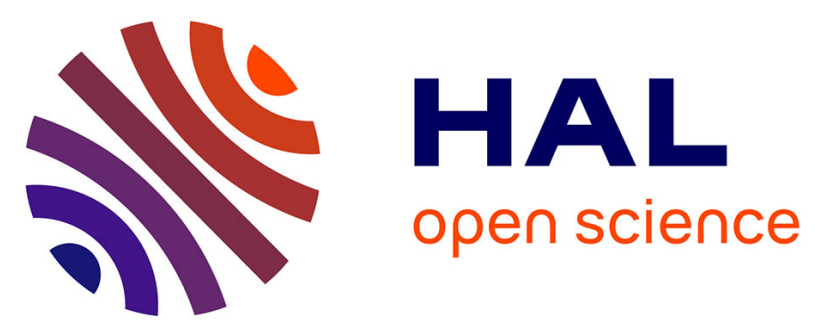

\title{
Dynamic and differential expression of the gonadal aromatase during the process of sexual differentiation in a novel transgenic cyp19a1a -eGFP zebrafish line
} Nathalie Hinfray, Frédéric Sohm, Morgane Caulier, Edith Chadili, Benjamin Piccini, Camille Torchy, Jean-Marc Porcher, Yann Guiguen, François Brion

\section{To cite this version:}

Nathalie Hinfray, Frédéric Sohm, Morgane Caulier, Edith Chadili, Benjamin Piccini, et al.. Dynamic and differential expression of the gonadal aromatase during the process of sexual differentiation in a novel transgenic cyp19a1a -eGFP zebrafish line. General and Comparative Endocrinology, 2018, 261, pp.179-189. 10.1016/j.ygcen.2017.06.014 . hal-01548670

\author{
HAL Id: hal-01548670 \\ https://hal.science/hal-01548670
}

Submitted on 3 Aug 2021

HAL is a multi-disciplinary open access archive for the deposit and dissemination of scientific research documents, whether they are published or not. The documents may come from teaching and research institutions in France or abroad, or from public or private research centers.
L'archive ouverte pluridisciplinaire HAL, est destinée au dépôt et à la diffusion de documents scientifiques de niveau recherche, publiés ou non, émanant des établissements d'enseignement et de recherche français ou étrangers, des laboratoires publics ou privés. 


\section{Dynamic and differential expression of the gonadal aromatase during the}

process of sexual differentiation in a novel transgenic cyp19a1a-eGFP zebrafish line

Hinfray Nathalie ${ }^{a}$, Sohm Frédéric ${ }^{b}$, Caulier Morganea ${ }^{a}$, Chadili Editha, Piccini Benjamin a, Torchy Camille $^{a}$, Porcher Jean-Marc ${ }^{a}$, Guiguen Yann ${ }^{c}$, Brion François ${ }^{a}$

a INERIS, Direction des Risques Chroniques, Pole VIVA, Unite d'ecotoxicologie in vitro et in vivo, BP2, 60550 Verneuil-en-Halatte, France

b UMS AMAGEN, CNRS, INRA, Université Paris-Saclay, 91198 Gif-sur-Yvette, France

c INRA, UR1037, Laboratoire de Physiologie et de Génomique des Poissons (LPGP), IFR140, OuestGenopole, F-35000 Rennes, France

Corresponding authors :

Dr Nathalie Hinfray

INERIS, Direction des risques chroniques, Pôle VIVA, Unité d'écotoxicologie in vitro et in vivo, BP2, 60550 Verneuil-en-Halatte, France

Phone : +33(0)3 44556969

Email : nathalie.hinfray@ineris.fr

Dr François Brion

INERIS, Direction des risques chroniques, Pôle VIVA, Unité d'écotoxicologie in vitro et in vivo, BP2, 60550 Verneuil-en-Halatte, France

Phone : +33(0)3 44556512

Email : francois.brion@ineris.fr 


\section{ABSTRACT}

In zebrafish, there exists a clear need for new tools to study sex differentiation dynamic and its perturbation by endocrine disrupting chemicals. In this context, we developed and characterized a novel transgenic zebrafish line expressing green fluorescent protein (GFP) under the control of the zebrafish cyp19a1a (gonadal aromatase) promoter. In most gonochoristic fish species including zebrafish, cyp19a1a, the enzyme responsible for the synthesis of estrogens, has been shown to play a critical role in the processes of reproduction and sexual differentiation. This novel cyp19a1a-eGFP transgenic line allowed a deeper characterization of expression and localization of cyp19a1a gene in zebrafish gonads both at the adult stage and during development. At the adult stage, GFP expression was higher in ovaries than in testis. We showed a perfect co-expression of GFP and endogenous Cyp19a1a protein in gonads that was mainly localized in the cytoplasm of peri-follicular cells in the ovary and of Leydig and germ cells in the testis. During development, GFP was expressed in all immature gonads of $20 \mathrm{dpf}$-old zebrafish. Then, GFP expression increased in early differentiated female at 30 and $35 \mathrm{dpf}$ to reach a high GFP intensity in well-differentiated ovaries at $40 \mathrm{dpf}$. On the contrary, males consistently displayed low GFP expression as compared to female whatever their stage of development, resulting in a clear dimorphic expression between both sexes. Interestingly, fish that undergoes ovary-to-testis transition (35 and $40 \mathrm{dpf}$ ) presented GFP levels similar to males or intermediate between females and males. In this transgenic line, our results confirm that cyp19a1a is expressed early during development, before the histological differentiation of the gonads, and that the down-regulation of cyp19a1a expression is likely responsible for the testicular differentiation. Moreover, we show that although cyp19a1a expression exhibits a clear dimorphic expression pattern in gonads during sexual differentiation, its expression persists whatever the sex suggesting that estradiol synthesis is important for gonadal development of both sexes. Monitoring the expression of GFP in control and exposed-fish will help determine the sensitivity of this transgenic line to EDCs and to refine mechanistic based-assays for the study of EDCs. In fine, this transgenic zebrafish line will be a useful tool to study physiological processes such as reproduction and sexual differentiation, and their perturbations by EDCs.

Key-Words: gonadal aromatase, transgenic zebrafish, sexual differentiation 


\section{Introduction}

Despite all the interest granted to zebrafish (Danio rerio) in the last few decades, the developmental genetic mechanisms controlling sexual fate in this species remain unclear (for review see (Liew and Orban, 2014; Nagabhushana and Mishra, 2016; Wilson et al., 2014). In natural wild strains of zebrafish, a strong sex determinant located at the end of the long (right) arm of chromosome 4 was recently discovered and this determinant is necessary but not sufficient for female development (Wilson et al., 2014). In contrast, laboratory strains most often lack this sex determination system (Wilson et al., 2014). Analysis of the available literature suggests that sex in those laboratory zebrafish strains would results of a complex interaction between genome (a polygenic sex determination system), primordial germ cells and environmental factors along with epigenetic factors possibly playing a role in mediating these processes (Liew and Orban, 2014; Luzio et al., 2015; Nagabhushana and Mishra, 2016).

During the sexual differentiation process, all male zebrafish develop a "juvenile ovary" which is later degenerated ("ovary-to-testis" transforming gonad) and transformed into a testis (Maack and Segner, 2003; Takahashi, 1977). While the identity of the gene(s) triggering sex determination/differentiation in zebrafish is not known at present, one common conserved factor in teleost sexual differentiation process is the involvement of estrogens-to-androgens ratios and P450 aromatase, the enzyme responsible for estrogens synthesis (for review see (Guiguen et al., 2010). In this regard, it is well-established that exposure to estrogens during the process of sexual differentiation resulted in a female biased sex-ratios at the adult stage (Baumann et al., 2014b; Brion et al., 2004; Holbech et al., 2006; Maack and Segner, 2004), while androgen and aromatase inhibitor exposures led to male biased sex-ratios (Baumann et al., 2014a; Fenske and Segner, 2004; McAllister and Kime, 2003; Morthorst et al., 2010; Uchida et al., 2004).

In most teleost fish, including zebrafish, the aromatase gene was duplicated giving rise to two different genes named cyp19a1a and cyp19a1b (Blazquez and Piferrer, 2004; Chang et al., 2005; Chiang et al., 2001a; Chiang et al., 2001b; Dalla Valle et al., 2002a; Dalla Valle et al., 2002b; Kobayashi et al., 2004; Tchoudakova and Callard, 1998). In zebrafish, the cyp19a1b gene is mainly expressed in the brain, specifically in radial glial cells, of both male and female zebrafish and is called the "brain aromatase" (Chiang et al., 2001a; Menuet et al., 2005; Pellegrini et al., 2007; Sawyer et al., 2006). The cyp19a1a gene is mainly expressed in the gonads, especially the ovary, and is also called the "gonadal aromatase" or "ovarian aromatase" (Chiang et al., 2001a; Sawyer et al., 2006). Current studies do support a decisive role of cyp19a1a gene in the phenotypic sex orientation of the zebrafish gonads. For instance, in vas::egfp transgenic zebrafish, cyp19a1a transcript levels clearly decreased in gonads undergoing subsequent testicular differentiation (Wang and Orban, 2007). Moreover, by producing loss-of-function mutations in zebrafish, Dranow et al. (2016) recently confirmed that Cyp19a1a is necessary for female sexual development in this species. Altogether, these studies support a critical role of Cyp19a1a in the ovarian differentiation and in the maintenance of the ovarian condition. 
Aromatase expression and/or activities have been shown to be disrupted by environmental endocrine disrupting chemicals (EDCs) (Hinfray et al., 2006b; Sanderson et al., 2002). For instance, several contaminants belonging to diverse class of chemical families can induce or inhibit aromatase in fish leading to biased sex ratios and/or disruption of reproductive outputs (Fenske and Segner, 2004; Kitano et al., 2000; Skolness et al., 2013; Sun et al., 2007; Uchida et al., 2004). In an attempt to further study the spatio-temporal expression and perturbation of aromatase A expression by EDCs, transgenic fish expressing a fluorescent reporter under the control of the cyp19a1a promoter would therefore be relevant biological models. In the present study, we developed and characterized a novel transgenic zebrafish line expressing green fluorescent protein (GFP) under the control of the zebrafish cyp19a1a promoter. Herein we report the expression and/or localization of cyp19a1a gene in adult zebrafish gonads both in control and in E2-exposed fish. Through fluorescence image analysis of GFP, the spatio-temporal expression of cyp19a1a was analyzed and semi-quantified along the process of histological sexual differentiation of the zebrafish and we confirmed that the downregulation of cyp19a1a gene expression is likely responsible for the testicular differentiation in zebrafish. The potential use of this novel transgenic zebrafish line for EDCs studies is also discussed.

\section{Materials and Methods}

\section{1. cyp19a1a-eGFP transgenic zebrafish line}

\subsubsection{Extraction of genomic DNA}

cyp19a1a promoter was isolated from genomic DNA of AB strain zebrafish. Genomic DNA was retrieved using a proteinase $\mathrm{K}$ protocol. Briefly, 30 hatched larvae were euthanized by an overdose of ethyl 3-aminobenzoate methanesulfonate (MS-222, Sigma-Aldrich, Lyon, France) and incubated in $250 \mu \mathrm{L}$ solution of Proteinase $\mathrm{K}$, (Merck, $10 \mathrm{mg} / \mathrm{mL}$ ) overnight at $56^{\circ} \mathrm{C}$. The solution was completed with $83.3 \mu \mathrm{L}$ of saturated $\mathrm{NaCl}$ solution (Sigma, $350 \mathrm{~g} / \mathrm{L}$ ) and centrifuged $(18,000 \mathrm{~g}, 20$ minutes). The liquid phase $(250 \mu \mathrm{L})$ was retrieved, precipitated using iso-propanol and washed twice in $70 \%$ ethanol. The pellet was resuspended in $30 \mu \mathrm{L}$ Tris buffer (Sigma, $10 \mathrm{mM}, \mathrm{pH}$ ) by incubation at $65^{\circ} \mathrm{C}$ for one hour and subsequently stored at $4^{\circ} \mathrm{C}$.

\subsubsection{Transgenic vector design}

We made use of transgenesis vector designed by AMAGEN as 'all fish species' transgenesis carriers. The sequences and important features of the cloning and transgenesis vectors are provided in Supplemental figures 1 to 4 . Briefly, the vectors were designed upon the multiple Gateway ${ }^{\circledR}$ cloning system described in (Roure et al., 2007) and carried 2 expression cassettes. The first cassette is an enhanced Cyan Fluorescent Protein (eCFP) coding sequence under the control of the zebrafish betacrystallin-b1 promoter (CrystBB1 cassette) which allows to follow the presence of the transgene in zebrafish from 48 hours post-fertilisation (hpf) onward (Emelyanov and Parinov, 2008). The second expression cassette allows to clone a promoter of interest (cyp19a1a in the present study) in front of an enhanced GFP (eGFP) reporter coding sequence. The insertion of the promoter is realised with a 
Gateway® Cloning kit using the attP3 and attP5 sites of the pEXP-GFP35H vector (Roure et al., 2007). The two cassettes are separated by a NOS terminator in order to reinforce transcriptional arrest after the CrystBB1 cassette (Emelyanov and Parinov, 2008). The transgenesis is realised through Involin (Thermes et al., 2002) using the two I-Scel sites present on each side of the transgene.

\subsubsection{PCR amplification of the promoter}

The $7.8 \mathrm{~kb}$ region upstream of the start codon of cyp19a1a was used as promoter to drive GFP expression. The PCR was designed in order to use the initiation codon and the Kozak consensus translation start of cyp19a1a fused to the eGFP coding sequence. The promoter was inserted using Gateway® cloning system (InVitrogen) as described in (Roure et al., 2007) into the pDONR35H (Supplemental figure 1). Briefly, a first fragment (8233 bp) was amplified from genomic DNA. We used the primers Cyp19a1a_Fwd and Cyp19a1a_Rev (Supplemental table 1) in a $50 \mu \mathrm{L}$ PCR mix (1 $\mu \mathrm{L}$ of genomic DNA (14 ng), KAPA Long Range HotStart DNA Polymerase (KAPA Biosystems, 1.25 U), 5x Kapa Long range buffer $(1 \mu \mathrm{L})$, dNTPs $\left(0.3 \mathrm{mM}\right.$ each), $\mathrm{MgCl}_{2}(1.75 \mathrm{mM})$ and $0.5 \mu \mathrm{M}$ of each primer) to run the first PCR: $1 \times\left(94^{\circ} \mathrm{C}-3 \mathrm{sec}\right) ; 35 \times\left(94^{\circ} \mathrm{C}-25 \mathrm{sec} ; 49^{\circ} \mathrm{C}-15 \mathrm{sec} ; 68^{\circ} \mathrm{C}-11 \mathrm{~min} 30 \mathrm{sec}\right) ; 1 \times\left(72^{\circ} \mathrm{C}\right.$ - $11 \mathrm{~min} 30 \mathrm{sec}$ ). This reaction yielded several products. A second reaction was used to improve specificity and insert the attB3 and attB5 Gateway recombination sites necessary for the cloning. The first PCR was diluted to the $10^{\text {th }}$ and $2 \mu \mathrm{L}$ were used as template of the nested PCR reaction. The primers Cyp19a1a_B3 and Cyp19a1a_B5 (Supplemental table 1) allowed to insert attB3 and attB5 recombination sites respectively at the 5' and 3' end of the promoter. The PCR conditions were identical to the first round except hybridization temperature, which was lowered to $47^{\circ} \mathrm{C}$. This reaction yielded a unique $7897 \mathrm{bp}$ fragment (7839 bp of promoter and $58 \mathrm{bp}$ of linkers). The attB3-attB5 tagged PCR fragment was purified using QIA quick PCR purification kit (Qiagen) according to manufacturer instructions. 50 femtomole of the PCR product and the vector pDONR35H (Supplemental figure 1) were used to carry out the cloning reaction using the BP clonase II kit (InVitrogen) to generate the pENT24-3 (Supplemental figure 2). The entry vector pENT24-3 was then recombined with pDEST35H (Supplemental figure 3) to generate the transgenesis vector pEXP24-3 (Supplemental figure 4). Both the pENT24-3 and the pEXP24-3 were fully sequenced (the sequences are provided in the supplemental figure 3 and 4 ).

\subsubsection{Generation of transgenic cyp19a1a-GFP zebrafish}

Transgenic zebrafish were generated by injecting the pEXP24-3 expression vector into one cell stage zebrafish eggs. Briefly, a solution containing pEXP24-3 maxiprep ( $15 \mathrm{ng} / \mu \mathrm{L}$ final) and I-Scel enzyme (Roche, $1 \mathrm{U} / \mu \mathrm{L}$ final) was injected into one cell stage eggs using borosilicate glass microcapillaries (GC100-10, Harvard Apparatus Ltd, UK), a femtojet injector (Eppendorf, Germany), a binocular loupe (Stemi Zv6, Zeiss, Germany) and Narishige (Japan) micromanipulator. In total, 789 eggs were injected in 3 sessions. At $48 \mathrm{hpf}$, the embryos were sorted according to eCFP expression in the lens under an epifluorescence dissecting microscope (MZFLIII Leica). Embryos which displayed differential expression from one eye to the other, strong mosaicism, weak or absence of CFP/GFP expression were eliminated. Roughly $20 \%$ of the injected eggs were selected for rearing (a batch of 77 
mild expressing embryos and a batch of 90 embryos displaying strong expression). After a rearing period of 4 months, 12 female and 4 male F0 adult fish were tested for their ability to transmit the transgene to their progeny. Among those, 4 females and 1 male transmitted the transgene to their progeny (Table 1). Progeny from each of these founders were further analysed for expression of GFP in the gonads. Two of the 5 lines did not express GFP at all. Each of the 3 other lines did express GFP according to a cyp19a1a compatible pattern albeit at levels varying from one line to the other (data not shown). The progeny of female 4 was selected as the most appropriate to our purpose (highest GFP expression intensity) and all further analysis were carried out using the progeny of this animal. The term cyp19a1a-eGFP thereafter refers exclusively to the lineage of this female 4.

Table 1: Transmission of the transgene from the five cyp19a1a-eGFP founders from generation $\mathbf{0}$ (F0) to generation 1 (F1)

\begin{tabular}{|l|l|l|l|}
\hline $\begin{array}{l}\text { ID and sex of the } \\
\text { transgenic } \\
\text { founders }(F 0)\end{array}$ & $\begin{array}{l}\text { Total number of } \\
\text { fertilized eggs }\end{array}$ & $\begin{array}{l}\text { Number of } \\
\text { transgenic } \\
\text { embryos (F1) }\end{array}$ & $\begin{array}{l}\% \text { positive } \\
\text { progeny }\end{array}$ \\
\hline $1 /$ female & 339 & 149 & 44 \\
\hline $2 /$ female & 51 & 11 & 22 \\
\hline $3 /$ female & 103 & 26 & 25 \\
\hline $4 /$ female & 383 & 80 & 21 \\
\hline $5 /$ male & 234 & 88 & 38 \\
\hline
\end{tabular}

\subsection{Zebrafish maintenance and reproduction}

Both transgenic (cyp19a1a-eGFP) and wild type (AB strain) zebrafish were raised in our laboratory facility at INERIS (Institut National de I'Environnement Industriel et des Risques, Verneuilen-Halatte, France). Zebrafish were maintained in $3.5 \mathrm{~L}$ aquaria in a recirculation system (Zebtec, Tecniplast, Buguggiate, Italy) on a $14: 10$ light:dark cycle at a temperature of $27.0 \pm 2.0^{\circ} \mathrm{C}$. Animal handling and experimentations were in accordance with the EU Directive 2010/63/EU for animal experiments.

Transgenic cyp19a1a-eGFP zebrafish were crossed with wild type zebrafish (AB strain) and fertilized eggs were harvested. Eggs were disinfected $5 \mathrm{~min}$ in water supplemented with $0.1 \%$ of commercial bleach (2.6\% of sodium hypochlorite). The CFP positive eggs/larvae were maintained in semi-static conditions until 8 days post fertilization (dpf) and then transferred in $3.5 \mathrm{~L}$ aquaria of the Zebtec system (around 25 larvae per liter) to grow.

To obtain the transgene transmission rate in the cyp19a1a-eGFP zebrafish line, 6 pairs of breeders each consisting of one transgenic and one wild type zebrafish, were allowed to reproduce. Transmission rate was determined by calculating the ratio of transgenic eggs over wild type eggs for each pair of breeders. 


\subsection{GFP fluorescence imaging and semi-quantification in the entire gonads of mature zebrafish}

Mature cyp19a1a-eGFP transgenic zebrafish were euthanized in ice-cold water (Strykowski and Schech, 2015; Wilson JM et al., 2009) before sectioning the spinal cord. The abdominal cavity was open to remove gut, liver, spleen and bile vesicle. Gonads were photographed with a Discovery V8 stereomicroscope equipped with an AxioCam Mrm camera (Carl Zeiss GmbH, Göttingen, Germany). GFP fluorescence in the entire gonads was then photographed and semi-quantified using a fluorescence imaging system IVIS Lumina II (Perkin Elmer, Courtaboeuf, France). All the pictures were taken in the same conditions (zoom, time of exposure, binning, F-Stop, excitation filter and emission filter). GFP fluorescence levels in the entire gonads were expressed as a measure of the photons incidence on the CCD camera (counts) and normalized by the area of the entire gonads (in squared millimeters, $\mathrm{mm}^{2}$ ). For each sex, 6 individuals were analyzed.

\subsection{Zebrafish exposure to estradiol and GFP fluorescence measurement}

Mature female cyp19a1a-eGFP zebrafish were exposed to $10 \mathrm{nM}$ E2 (Sigma-Aldrich) or to solvent alone (dimethyl sulfoxide (DMSO), 0.00001\%; Sigma-Aldrich, Lyon, France) during 7 days. Fish were exposed under semi-static conditions in $4 \mathrm{~L}$ tanks with $100 \%$ water renewal every day (temperature: $28 \pm 1{ }^{\circ} \mathrm{C}$; $\mathrm{pH}$ : $8.0 \pm 0.2$; dissolved oxygen: $6.6 \pm 0.3 \mathrm{mg} / \mathrm{l}$; conductivity: $275 \pm 43$ $\mathrm{mS} / \mathrm{cm}$ ). Exposure was performed in one tank per condition, each containing four female fish. At the end of exposure, zebrafish were euthanized and gonads were removed and fixed $48 \mathrm{~h}$ in $4 \%$ paraformaldehyde in saline phosphate buffer (PBS) at $4^{\circ} \mathrm{C}$. After rinsing in PBS, samples were successively transferred to $10 \%, 20 \%$ and $30 \%$ sucrose-PBS solutions at $4{ }^{\circ} \mathrm{C}$ and finally embedded in Neg-50 cryo-compound (Microm Microtech, France). Frozen sections of gonads were performed at 12 $\mu \mathrm{m}$ thick. Slides were rinsed in PBS to remove Neg-50 cryo-compound and coverslipped using a fluorescence mounting medium (DAKO, France).

GFP fluorescence was observed directly on cryo-sections using a Zeiss Axiolmager Z1 fluorescence microscope equipped with an AxioCam Mrm camera (Carl Zeiss GmbH, Göttingen, Germany). Micrographs were taken using the Axiovision Imaging software. Semi-quantification of the fluorescence of GFP of DMSO- and E2-exposed fish ovaries was performed on micrographs taken with the same magnification (x2.5 objective) and time of exposure for all individuals. For each experimental condition, 4 fish were used and 4 micrographs were analyzed per fish. Fluorescence was measured as integrated density (IntDen, ImageJ software), i.e. the sum of the grey values of all the pixels in the region of interest. The IntDen values were then normalized to the total area of tissue on the micrograph.

\subsection{Fluorescence immunohistochemistry}

cyp19a1a-eGFP transgenic/wild type $A B$ zebrafish were euthanized and gonads were removed and fixed in modified Davidson's fixative (22\% v/v formaldehyde $37-40 \% ; 11.5 \% \mathrm{v} / \mathrm{v}$ glacial 
acetic acid; $33 \% \mathrm{v} / \mathrm{v}$ ethyl alcohol $95 \% ; 33.5 \% \mathrm{v} / \mathrm{v}$ distilled water) for 48 hours at room temperature. After fixation, samples were dehydrated in ethanol, cleared in toluene and embedded in paraffin, according to conventional procedures. Zebrafish gonads were sectioned at $5 \mu \mathrm{m}$.

Immunohistochemistry was then carried out using an In situ Pro VSI robotic station (Intavis, Köln, Germany, distributed in France by Proteigene) as previously described in (Hinfray et al., 2013). For GFP localization, a primary antibody produced in goat was used at a dilution of 1:600 (polyclonal goat anti-GFP, Abcam, Paris, France, reference number: ab5450). For Cyp19a1a localization, a primary antibody produced in rabbit was used at a dilution of 1:600 (polyclonal rabbit anti-zfCyp19a1a, AnaSpec, Fremont, USA, distributed in France by Tebu-Bio, reference number: 55474s). For colocalization experiment, slides were incubated with a mix of these two antibodies at a dilution of 1:600. These primary antibodies have been already used successfully in zebrafish (Hinfray et al., 2013; Klein et al., 2011). Slides were then incubated with a donkey anti-goat antibody coupled to AlexaFluor $\AA^{4} 488$ or a mix of donkey anti-goat coupled to AlexaFluor ${ }^{\circledR} 488$ and donkey anti-rabbit coupled to AlexaFluor ${ }^{\circledR} 594$ antibodies (Invitrogen, Praisley, UK) at a dilution of 1:200. Nuclei were revealed with Hoechst 33342 . The specificity of the immunoreactivity was controlled by processing adjacent sections without primary antibody or with only one primary antibody and the two secondary antibodies. The specificity of the anti-GFP antibody was controlled by performing immunohistochemistry on wild type zebrafish gonad sections. The specificity of the anti-Cyp19a1a antibody was also controlled by performing immunohistochemistry with the anti-Cyp19a1a antibody pre-incubated with $100 \mu \mathrm{g} / \mathrm{ml}$ of the synthetic peptide. In these negative controls, no cross-reactivity was seen (Supplemental Figure 5).

GFP fluorescence was observed and photographed using an FV1000 confocal microscope (Olympus, Rungis, France). Acquisition parameters (time of exposure, laser intensity...) were optimized for each micrograph independently.

\subsection{Gonadal GFP fluorescence during the process of sexual differentiation}

cyp19a1a-eGFP transgenic zebrafish larvae were sampled at 20,30, 35 and $40 \mathrm{dpf}(\mathrm{n}=8$ at $20 \mathrm{dpf}$ and 15 at the other sampling time). The larvae were euthanized with an overdose of MS-222. Entire larvae (20 dpf) or larvae without their heads $(30,35,40 \mathrm{dpf})$ were fixed $48 \mathrm{~h}$ in $4 \%$ paraformaldehyde in PBS at $4^{\circ} \mathrm{C}$. After rinsing in PBS, larvae were successively transferred to $10 \%$, $20 \%$ and $30 \%$ sucrose-PBS solutions at $4{ }^{\circ} \mathrm{C}$, embedded in Neg-50 cryo-compound and sectioned transversally at $12 \mu \mathrm{m}$ thick. Slides were rinsed in PBS and coverslipped using the DAKO fluorescence mounting medium.

GFP fluorescence was observed directly on cryo-sections using a Zeiss Axiolmager Z1 fluorescence microscope equipped with an AxioCam Mrm camera and combined with an ApoTome (Carl Zeiss $\mathrm{GmbH}$, Göttingen, Germany). Illustration micrographs were taken using the ApoTome system and Axiovision Imaging software. Relative quantification of the fluorescence of GFP of the larvae gonads was performed on micrographs taken without the ApoTome system but with the same magnification (x10 objective) and time of exposure for all individuals whatever the sex and stage of 
development. For each larva, 3 to 4 micrographs of the entire gonads were analyzed. GFP fluorescence was measured as integrated density (IntDen, ImageJ software).

\subsection{Whole Mount In Situ Hybridization (WMISH) on early stage zebrafish}

Wild type $A B$ zebrafish larvae were sampled at $20 \mathrm{dpf}$, euthanized with an overdose of MS222 and fixed in $4 \%$ paraformaldehyde $\left(24\right.$ hours, $\left.4^{\circ} \mathrm{C}\right)$. After fixation, larvae were rinced 3 times (PBS-Tween 20 0.1\%), progressively dehydrated in methanol and stored in methanol 100\% until WMISH.

RNA probes were produced from PCR product obtained by following amplification of the IRBOp991C1282D clone (Source Bioscience, UK) containing the zebrafish cyp19a1a cDNA. Colonies containing plasmid were isolated with $50 \mu \mathrm{g} / \mathrm{ml}$ ampicillin. Plasmids were purified using PureLink ${ }^{\circledR}$ Quick Plasmid Miniprep kit (ThermoFisher Scientific) according to manufacturer's protocol. PCR amplification was carried out on $2 \mathrm{ng}$ of plasmid as follow: 1 cycle at $94^{\circ} \mathrm{C}$ for $1 \mathrm{~min}, 35 \mathrm{cycles}$ at $\left(94^{\circ} \mathrm{C}\right.$ for $40 \mathrm{sec}, 46^{\circ} \mathrm{C}$ for $40 \mathrm{sec}$ and $72^{\circ} \mathrm{C}$ for $\left.2 \mathrm{~min}\right)$, and $1 \mathrm{cycle}$ at $72^{\circ} \mathrm{C}$ for $10 \mathrm{~min}$; using the following primers 5'-TAA TAC GAC TCA CTA TAG GG-3' and 5'-CAA TTA ACC CTC ACT AAA GG-3'. Ten nanograms of the resulting PCR product was then used as template for digoxygenin-labeled RNA probes synthesis using DIG RNA labeling mix (Roche, France) and T3 or T7 RNA polymerase (Roche, France) following standard protocols. Labeled RNA probes were then purified using Micro Bio-Spin 30 Columns (BioRad, France) and stored at $-80^{\circ} \mathrm{C}$ until use.

WMISH was carried out using an In Situ Pro (Intavis) robotic station as described in Yano et al. (2011) with minor modifications. Briefly, $20 \mathrm{dpf}$ zebrafish larvae were rehydrated, permeabilized by proteinase $\mathrm{K}$ treatment $(25 \mu \mathrm{g} / \mathrm{ml}, 35 \mathrm{~min}$, room temperature), and post-fixed (4\% paraformaldehyde and $0.8 \%$ glutaraldehyde, $20 \mathrm{~min}$ ). Pre-hybridization and hybridization medium contained $50 \%$ formamide, 5 X SCC, $0.1 \%$ Tween $20,0.005 \%$ heparin, $0.1 \mathrm{mg} / \mathrm{ml}$ tRNA. Hybridization was carried out at $37^{\circ} \mathrm{C}$ for 16 hours. After post-hybridization washes, larvae were incubated in blocking buffer for 1 hour (Triton 1\%, Tween 20 0.2\%, 2\% serum/PBS) before addition of the alkaline phosphatase coupled anti-digoxygenin antibody (1:2,000, Roche Diagnostics Corp) for 6 hours. After washes, color reaction was performed in the presence of NBT/BCIP (Roche). After WMISH, larvae were dehydrated and embedded in paraffin according to conventional procedures, and sectioned at $5 \mu \mathrm{m}$.

\subsection{Statistics}

To compare mean fluorescence data (female vs male, DMSO vs E2 and GFP expression in the different sexes and stages of development), a non-parametric Wilcoxon's test was performed, where it was possible, using $R_{T M}$ ( $R$ software, $R$ development Core Team). Differences between groups were considered to be significant if $p<0.05\left({ }^{*}\right)$ and $p<0.01\left(^{* *}\right)$.

\section{Results}




\subsection{Transmission of the transgene in the cyp19a1a-eGFP transgenic zebrafish line}

Adult cyp19a1a-eGFP heterozygote transgenic zebrafish ( $\mathrm{N}=3$ males and 3 females) were allowed to reproduce with wild type $A B$-strain zebrafish to determine the transmission rate of the transgene from generation 3 to generation 4 . Transgenic larvae were identified by the presence of CFP fluorescence in the eyes. Transmission of the transgene occurred for the 6 transgenic fish and the transmission rate was about $50 \%$ for each breeder fish (Table 2) suggesting a unique insertion of the transgene in these transgenic fish. Besides the unique insertion of the transgene, this cyp19a1aeGFP line also displays interesting features such as being a stable transgenic line with the possibility to select transgenic eggs as soon as $48 \mathrm{hpf}$.

Table 2: Transmission rate of the transgene from generation $3(F 3)$ to generation $4(F 4)$ in the cyp19a1a-GFP transgenic zebrafish line. 6 transgenic zebrafish were cross-breaded with wild type $A B$ zebrafish.

\begin{tabular}{|l|l|l|l|}
\hline $\begin{array}{l}\text { ID and sex of the } \\
\text { transgenic fish (F3) }\end{array}$ & $\begin{array}{l}\text { Total number of } \\
\text { fertilized eggs }\end{array}$ & $\begin{array}{l}\text { Number of transgenic } \\
\text { embryos (F4) }\end{array}$ & $\begin{array}{l}\text { Transmission rate } \\
(\%)\end{array}$ \\
\hline $1 /$ female & 388 & 196 & 50.5 \\
\hline 2 / female & 187 & 87 & 46.5 \\
\hline 3 / female & 216 & 104 & 48.1 \\
\hline 4 / male & 203 & 93 & 45.8 \\
\hline 5 / male & 183 & 87 & 47.5 \\
\hline 6 / male & 123 & 64 & 52.0 \\
\hline
\end{tabular}

3.2. GFP expression, localization and regulation in the cyp19a1a-eGFP transgenic zebrafish line

The expression of GFP in mature zebrafish gonads was determined by fluorescence imaging and relative quantification (Figure 1). In all male and female fish studied, GFP expression was higher in ovaries than in testis (Figure 1A, B) with a 9-fold higher expression in ovaries compared to testis (Figure 1B). 
A
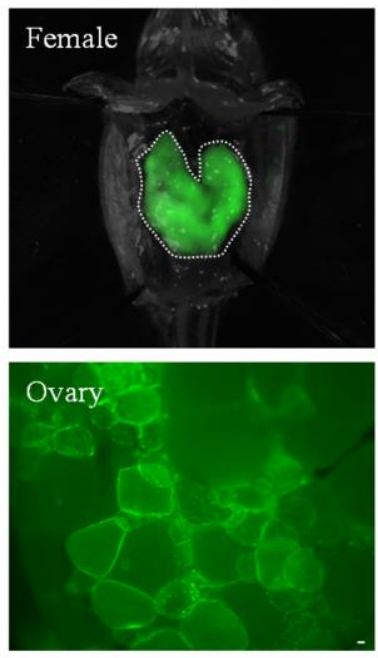

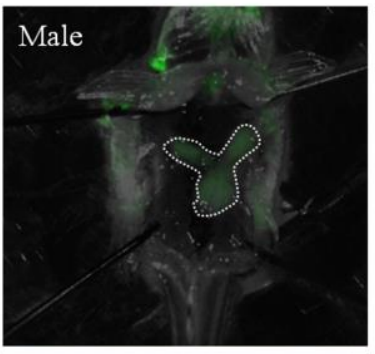

Testis

$\mathrm{B}$

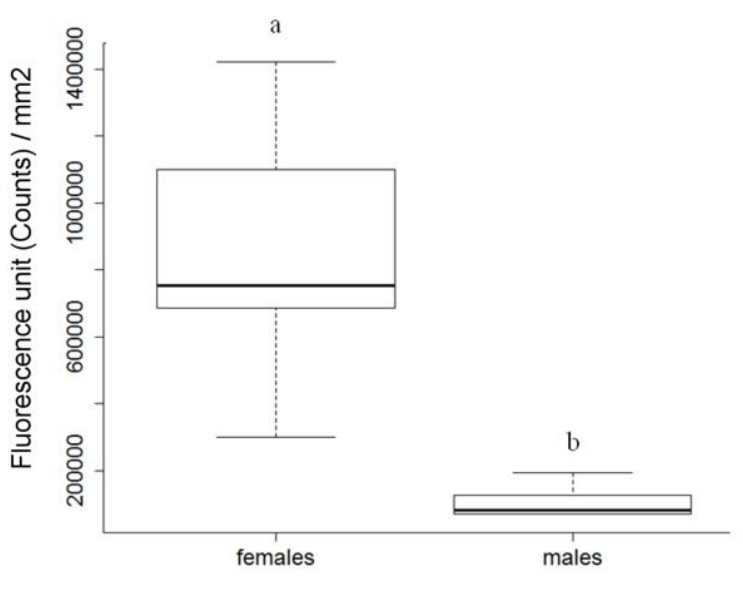

Figure 1: Relative quantification of GFP fluorescence in mature zebrafish gonads of the cyp19a1a-GFP transgenic line. (A) Imaging of GFP fluorescence (green) in the entire gonads using a fluorescence imaging system IVIS Lumina II from Perkin Elmer (Top) and a Discovery V8 stereomicroscope from Carl Zeiss (Bottom). Scale bars $=50 \mu \mathrm{m}(\mathrm{B})$ Relative quantification of GFP fluorescence in the entire gonads of females $(\mathrm{N}=6)$ and males $(\mathrm{N}=6)$ with the IVIS Lumina II system. Results are expressed as the median value (bars in the middle of the box), upper quartile (upper edge of the box) and lower quartile (lower edge of the box). Different letters indicate a statistically significant difference between the groups (Wilcoxon's Test, $p<0.05$ ).

At the cellular level, in $55 \mathrm{dpf}$-old zebrafish ovary, GFP was mainly localized to the cytoplasm of peri-follicular cells surrounding previtellogenic oocytes, with a slight fluorescence also in the cytoplasm of oocytes (Figure 2 A,B). In mature ovary, GFP was immunolocalized also in the cytoplasm of peri-follicular cells (theca and granulosa cells) surrounding oocytes from a slight expression around some previtellogenic oocytes to a strong expression around vitellogenic oocytes (Figure $2 \mathrm{C}-\mathrm{G}$ ). A slight GFP expression was also noticed in oocyte cytoplasms. In mature testis, GFP localized to the cytoplasm of both Leydig cells and germ cells (Figure $2 \mathrm{H}$ ). 

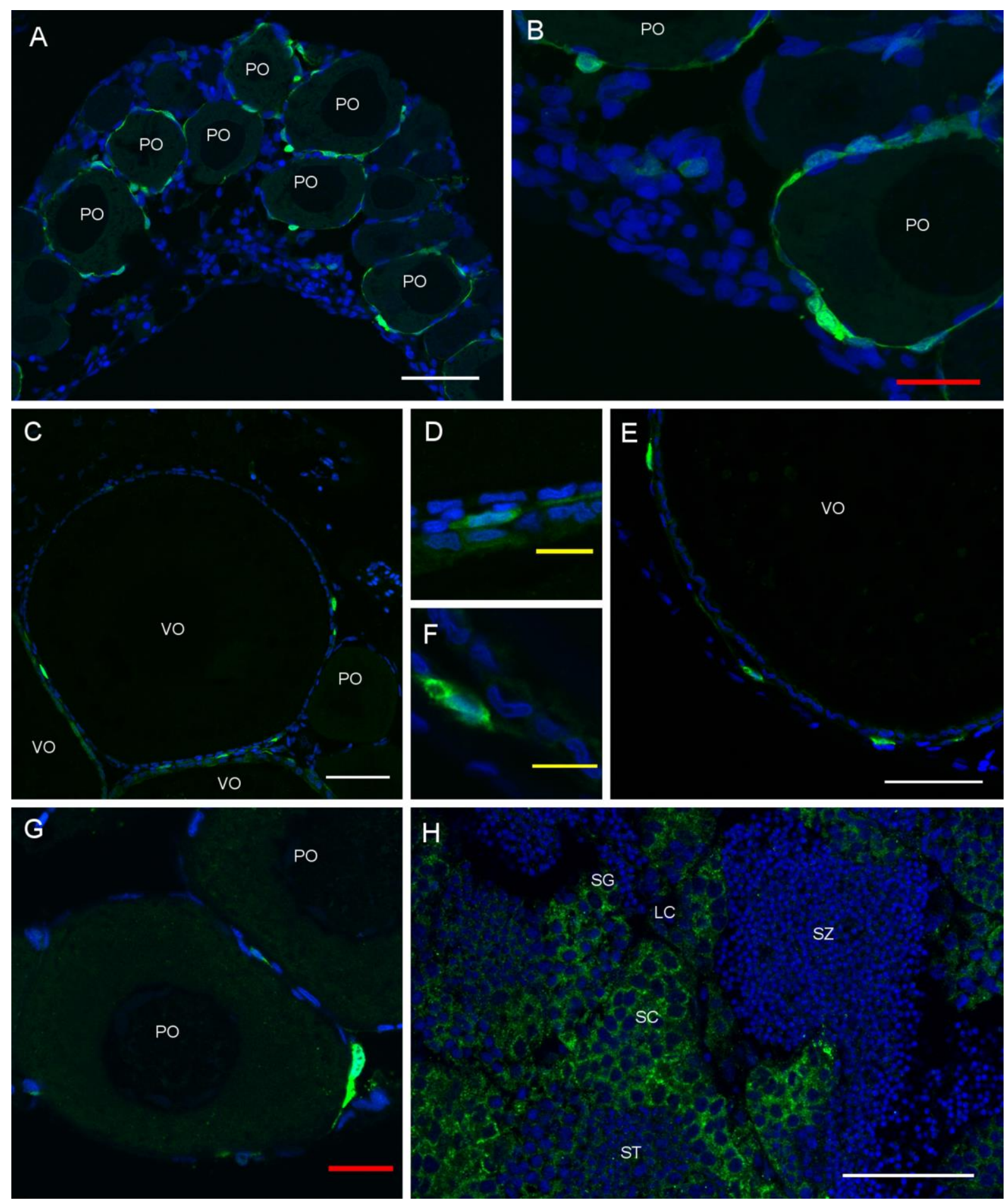

Figure 2: GFP fluorescence localization in zebrafish gonads of the cyp19a1a-GFP transgenic line. In 55 days post fertilization (dpf) zebrafish, GFP was localized to the cytoplasm of peri-follicular cells surrounding pre-vitellogenic oocytes $(A, B)$. In mature ovary of adult zebrafish, GFP is mainly immunolocalized in the cytoplasm of peri-follicular cells (granulosa and thecal cells) surrounding vitellogenic oocytes and some pre-vitellogenic oocytes (C-G). (D) Enlargement of peri-follicular cells from micrograph C. (F) Enlargement of peri-follicular cells from micrograph E. In mature testis, GFP localized to the cytoplasm of both Leydig cells and germ cells $(\mathrm{H})$. Imaging was realized with an Olympus FV1000 confocal microscope. Acquisition parameters were optimized for each micrograph independently. Nuclei are in blue. PO: pre-vitellogenic oocyte; VO: vitellogenic oocyte; LC: Leydig cells; SC: spermatocytes; SG: spermatogonia; ST: spermatids; SZ: spermatozoa. White bars: $50 \mu \mathrm{m}$; Red bar: $20 \mu \mathrm{m}$; Yellow bars: $10 \mu \mathrm{m}$. 
To assess whether GFP is indeed expressed in Cyp19a1a-expressing cells, sections of cyp19a1a-eGFP transgenic fish gonads were immunolabeled both with anti-GFP and anti-Cyp19a1a antibodies. In both mature ovary and testis, a perfect co-localization of GFP and Cyp19a1a proteins was observed (Figure 3).
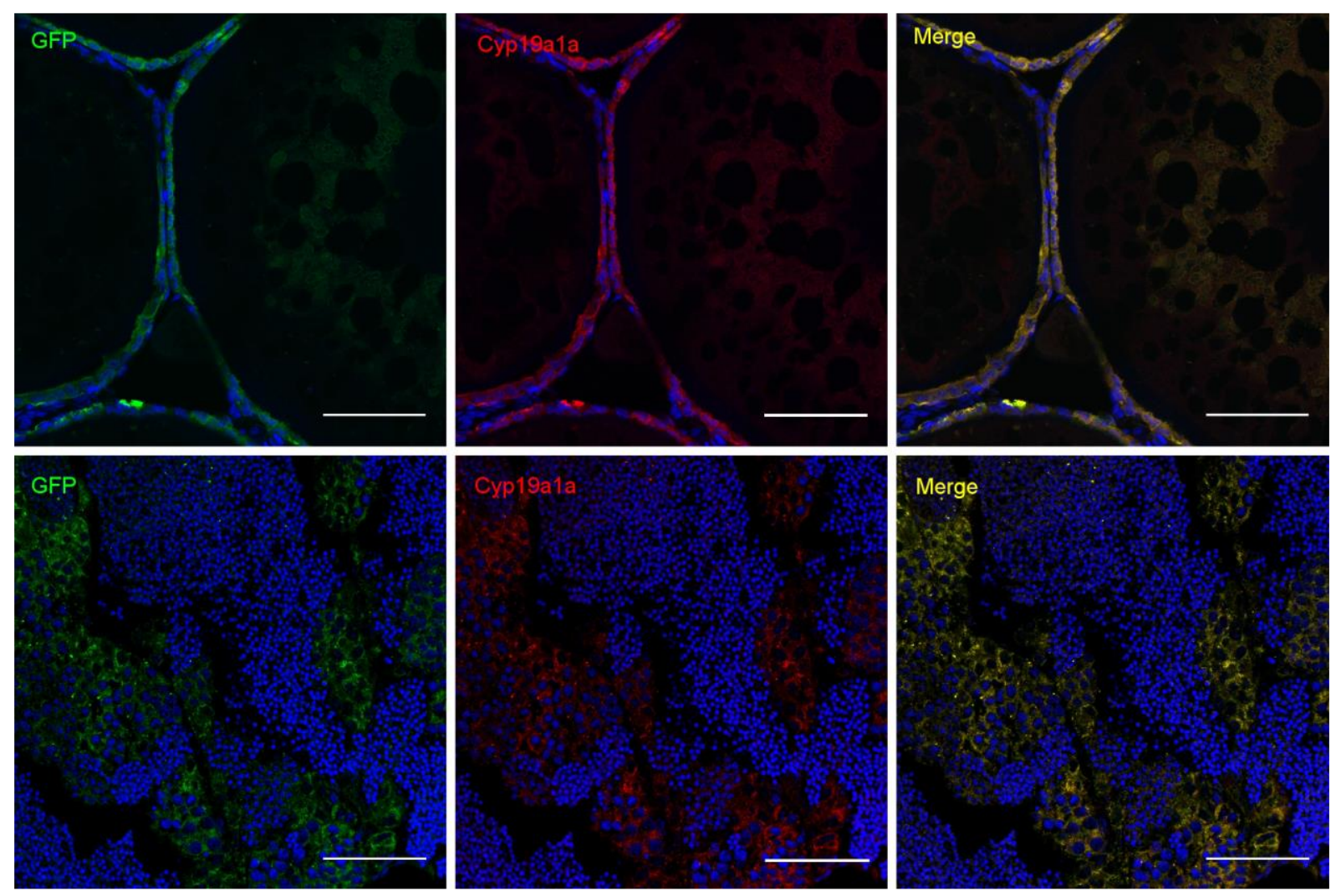

Figure 3: Co-localization of GFP and Cyp19a1a in mature zebrafish gonads of the cyp19a1aGFP transgenic line. GFP (green) and Cyp19a1a (Red) proteins were immunolocalized both in ovaries (Top) and testis (Bottom). The merge of the two is showed in yellow. Nuclei are in blue. Imaging was realized with an Olympus confocal microscope FV1000. Acquisition parameters were optimized for each sex independently. White bars: $50 \mu \mathrm{m}$.

In a previous study, we showed that exposure to 10nM of E2 for 7 days inhibited cyp19a1a gene expression in wild type zebrafish ovary (Hinfray et al., 2006a). In the present study, GFP fluorescence in adult cyp19a1a-eGFP transgenic zebrafish ovaries was also inhibited by exposure to E2 (10nM, 7 days) (Figure 4), indicating that GFP expression regulation by E2 in transgenic zebrafish is similar to that of cyp19a1a gene in wild type zebrafish. 


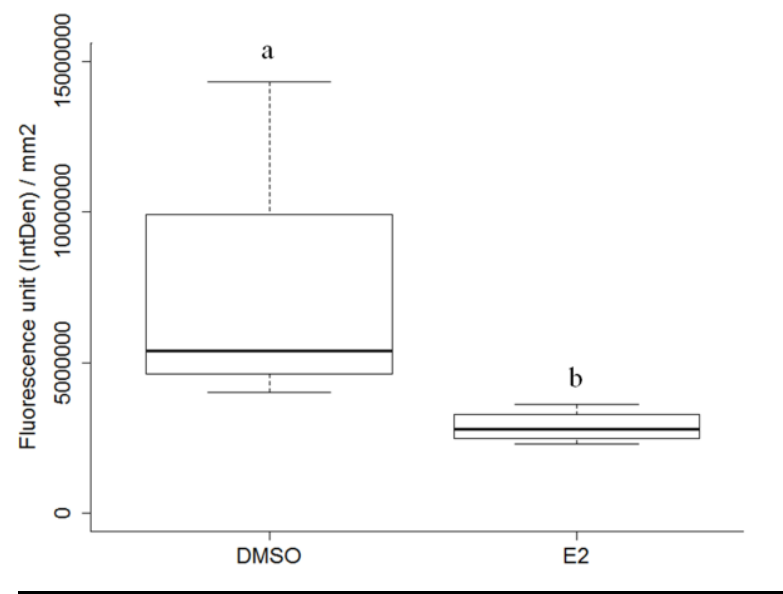

Figure 4: Relative quantification of GFP fluorescence in mature cyp19a1a-GFP transgenic zebrafish ovaries after a 7-days exposure to $10 \mathrm{nM}$ of Estradiol (E2) or solvent (DMSO). Results are expressed as the median value (bars in the middle of the box), upper quartile (upper edge of the box) and lower quartile (lower edge of the box). For each individual ( $n=4$ individuals / condition), four micrographs of ovarian sections were analyzed. Different letters indicate a statistically significant difference between the groups (Wilcoxon's Test, $p<0.05$ ).

\subsection{Spatio-temporal expression of GFP in cyp19a1a-eGFP transgenic zebrafish gonads during development}

The spatio-temporal expression of cyp19a1a gene was studied during the process of sexual differentiation using GFP fluorescence imaging and semi-quantification in gonad sections of transgenic zebrafish between 20 and $40 \mathrm{dpf}$ (Figure 5, 6 and 7). A slight expression of GFP was observed in all immature gonads of $20 \mathrm{dpf}$-old zebrafish and tends to increase in 30 and $35 \mathrm{dpf}$-old zebrafish immature gonads (Figure 5). In immature gonads of 20 dpf cyp19a1a-eGFP transgenic zebrafish, GFP and Cyp19a1a expression was localized in the cytoplasm of all cell types (Figure $6 \mathrm{~A}, \mathrm{~B}$ ). At the same stage of development, cyp19a1a mRNA was also localized in the cytoplasm of all cell types of wild type zebrafish gonads (Figure $6 \mathrm{C}$ ). In females (from $30 \mathrm{dpf}$ ), GFP expression was higher than in immature gonads and its expression increased to reach high GFP intensity in well-differentiated ovaries of $40 \mathrm{dpf}$-old zebrafish (Figure 5). At this stage, GFP was mainly observed in the cytoplasm of peri-follicular cells but a slight fluorescence was also noticed in the cytoplasm of oocytes (Figure 7). On the contrary, males consistently displayed low GFP expression as compared to females whatever their stage of development, resulting in a clear dimorphic expression between both sexes (Figure 5). At $40 \mathrm{dpf}$, GFP was localized in the cytoplasm of several isolated cells distributed in the entire testis (Figure 7). Interestingly, gonads of fish that undergo ovary-to-testis transition (35 and $40 \mathrm{dpf}$ ) presented GFP levels similar to males or intermediate between females and males (Figure 5). In this ovary-to-testis transition gonads, GFP is expressed in cells at the periphery of the gonad while no GFP was found in the peri-follicular cells surrounding remaining oocytes (Figure 7). 


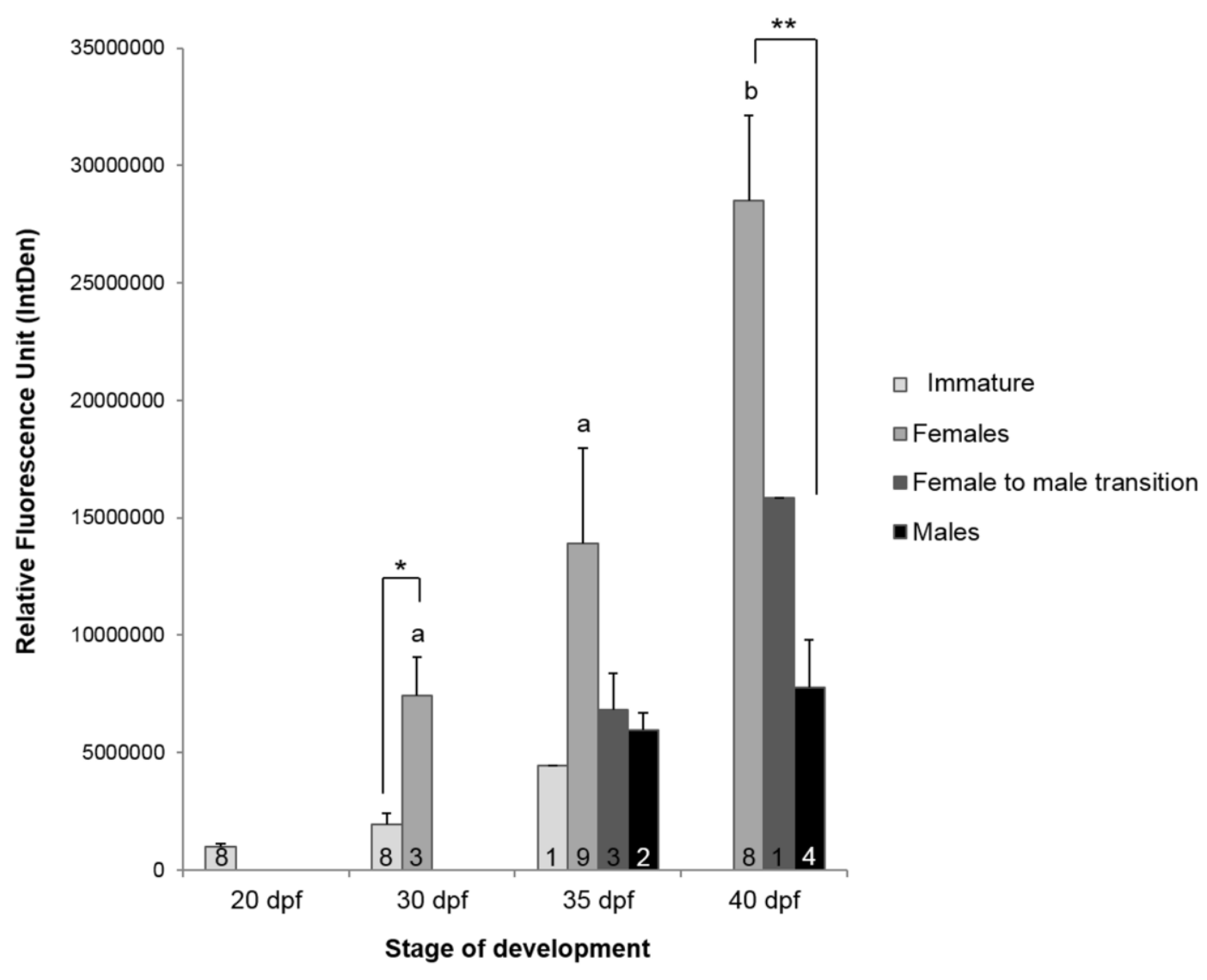

Figure 5: Relative quantification of GFP fluorescence in gonads of cyp19a1a-GFP transgenic zebrafish during development. Results are expressed as mean \pm S.E.M. Number of fish analyzed is indicated in the bars of the histogram and 3-4 sections were analyzed for each fish. Different letters indicate statistically significant differences across development for one sex (Wilcoxon's test, $p<0.05$ ). * and ${ }^{* *}$ indicate statistically significant differences between sexes within one stage of development (Wilcoxon's test, ${ }^{*} p<0.05,{ }^{* *} p<0.01$ ). dpf: days post fertilization.
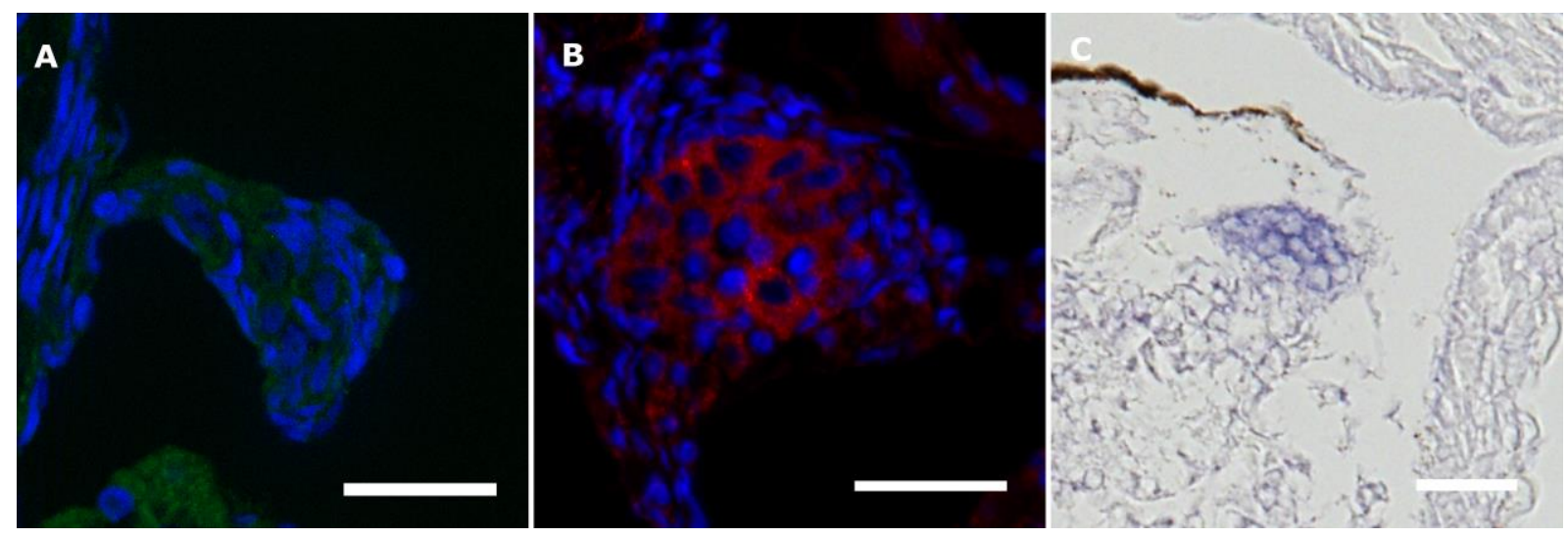

Figure 6: Localization of GFP and cyp19a1a protein and mRNA in $20 \mathrm{dpf}$ gonads of zebrafish. A: Imaging of GFP fluorescence (green) was realized directly on cryo-sections of cyp19a1a-GFP transgenic zebrafish gonads. B: Cyp19a1a (Red) proteins were immunolocalized using the antiCyp19a1a antibody in cyp19a1a-GFP transgenic zebrafish gonads. C: cyp19a1a mRNA was localized by whole mount in situ hybridization in wild type zebrafish gonads. Fluorescence imaging was realized using a Zeiss Axiolmager Z1 fluorescence microscope equipped with a MRm5 camera combined with an ApoTome. Nuclei are in blue. Acquisition parameters were optimized for each micrograph independently. Transmitted light imaging was performed using a Nikon 90i microscope equipped with a Nikon DS Ri1 camera. dpf: days post fertilization. Scale bars: $20 \mu \mathrm{m}$. 

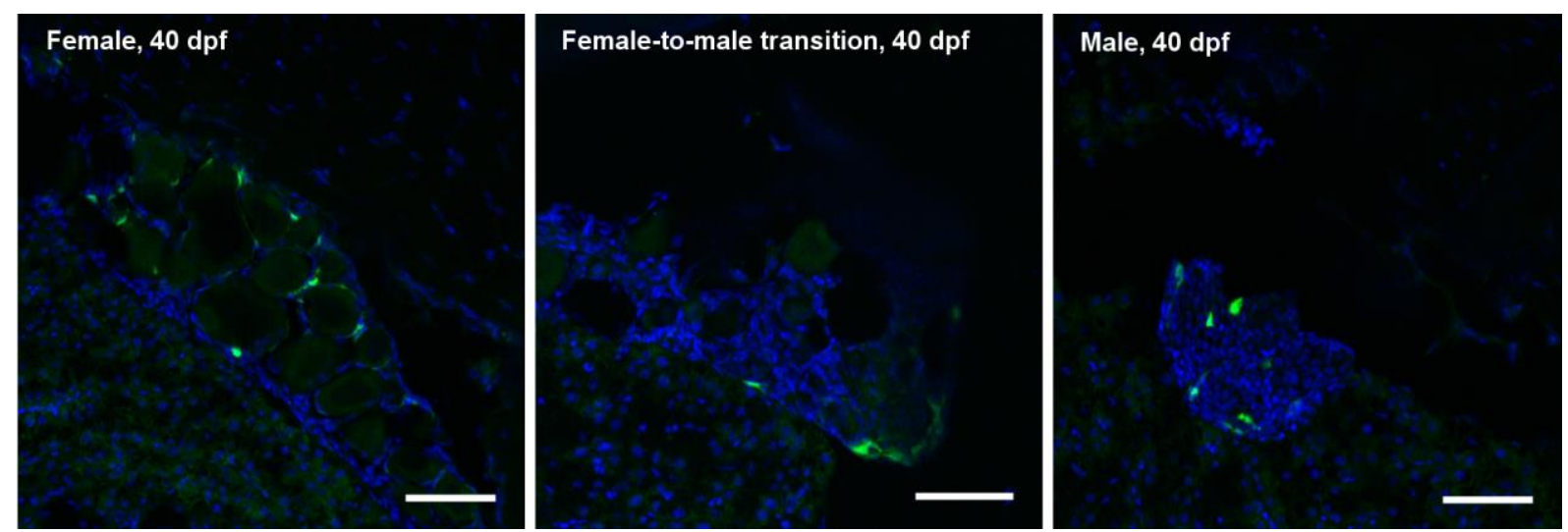

Figure 7: Localization of GFP fluorescence in 40 dpf gonads of cyp19a1a-GFP transgenic zebrafish. Imaging of GFP fluorescence (green) was realized directly on cryo-sections using a Zeiss Axiolmager Z1 fluorescence microscope equipped with an MRm5 camera combined with an ApoTome. Acquisition parameters were optimized for each micrograph independently. Nuclei are in blue. dpf: days post fertilization. Scale bars: $50 \mu \mathrm{m}$

\section{Discussion}

\subsection{The cyp19a1a gene is expressed both in female and male gonads}

In the present study, we established a novel and useful transgenic line of zebrafish expressing GFP under the control of the zebrafish cyp19a1a gene promoter. In these transgenic animals, GFP was expressed in both male and female gonads with a 9-fold higher fluorescence level in ovaries, which is consistent with the crucial production of estrogens in females with important roles in oocyte growth and hepatic vitellogenin synthesis (Nagahama, 1994). This dimorphic GFP expression is in agreement with the higher expression of cyp19a1a gene (Fenske and Segner, 2004; Sawyer et al., 2006; Trant et al., 2001) and Cyp19a1a protein (Caulier et al., 2015) measured in wild type zebrafish ovaries compared to testis as well as with the higher circulating concentrations of estradiol in female zebrafish (Deng et al., 2010; Ji et al., 2013; Liu et al., 2013; Liu et al., 2012; Ma et al., 2012; Yu et al., 2014).

In ovaries, GFP was detected in the cytoplasm of peri-follicular cells (thecal and granulosa cells) surrounding both previtellogenic and vitellogenic oocytes with a stronger expression around vitellogenic oocytes. This pattern of expression is similar to the wild type pattern of cyp19a1a gene and its protein in zebrafish ovaries (Caulier et al., 2015; Goto-Kazeto et al., 2004; Rodriguez-Mari et al., 2005; Wang and Orban, 2007) and to another recently developed cyp19a1a-EGFP transgenic zebrafish (Dranow et al., 2016). A slight GFP fluorescence was also measured in the cytoplasm of oocytes. In other fish species such as medaka, rainbow trout, air-breathing catfish or gilthead seabream, cyp19a1a gene or its protein product have already been detected in the germinal compartment, i.e. the ooplasm (Gohin et al., 2011; Park et al., 2008; Raghuveer et al., 2011; Zapater et al., 2012), and proved to be functional in rainbow trout late vitellogenic oocytes (Gohin et al., 2011). 
In zebrafish, however, due to the presence of Cyp19a1b protein in the ooplasm (Caulier et al., 2015), the specific functionality of Cyp19a1a protein remains difficult to demonstrate in this compartment.

In testis, both Leydig cells and germ cells expressed GFP. In wild type zebrafish testis, while cyp19a1a gene expression has been measured, no mRNA localization was achieved (Hinfray et al., 2006a; Rodriguez-Mari et al., 2005; Sawyer et al., 2006; Wang and Orban, 2007). Nevertheless, this GFP localization to Leydig and germ cells is consistent with our previous studies showing the same pattern of localization of Cyp19a1a protein in wild type zebrafish testis (Caulier et al., 2015; Hinfray et al., 2013). In other teleosts, cyp19a1a gene and/or protein have been detected in both Leydig cells (Guiguen et al., 2010; Kobayashi et al., 2004; Kotula-Balak et al., 2008; Vinas and Piferrer, 2008; von Schalburg et al., 2013) and germ cells (Kotula-Balak et al., 2008; Vinas and Piferrer, 2008; von Schalburg et al., 2013) of the testis. All these data argue for a role of estrogens in fish testis that might be linked to the involvement of estradiol in spermatogonial stem cell renewal in fish (Miura et al., 1999; Miura et al., 2003).

Altogether, expression of GFP in the cyp19a1a-eGFP transgenic zebrafish is consistent with the expression of endogenous cyp19a1a gene expression in the gonads of wild type zebrafish. The presence of the CrystBB1-CFP reporter allows for early selection of transgenic eggs and does not seem to disturb the expression of the cyp19a1a promoter. These combine to make of this line a useful and powerful tool to monitor cyp19a1a expression and determine its precise role in different physiological processes.

\subsection{Down-regulation of gonadal cyp19a1a expression is likely responsible for testicular differentiation}

In the present study, the cyp19a1a-eGFP transgenic zebrafish was used to analyze the spatiotemporal expression of the cyp19a1a gene in individual gonads during the sexual differentiation process. At the early stages (from $20 \mathrm{dpf}$ to $35 \mathrm{dpf}$ ), a slight GFP fluorescence was detected in all cell types of all immature gonads. At $20 \mathrm{dpf}$, cyp19a1a mRNA and protein were also localized in the cytoplasm of all cell types of zebrafish gonads. This early expression of the cyp19a1a gene has already been evidenced in wild type zebrafish trunks by RT-PCR (Fenske and Segner, 2004; Wang and Orban, 2007) and individual gonads by in situ hybridization (Rodriguez-Mari et al., 2005), and also in gonads of the TgBAC(cyp19a1a:EGFP) uc44 zebrafish of $13 \mathrm{dpf}$ (Dranow et al., 2016). However, contrary to the localization of cyp19a1a mRNA by in situ hybridization which has been shown to be restricted to some regions of the immature gonads (Rodriguez-Mari et al., 2005), a wider distribution of GFP and Cyp19a1a protein within the cyp19a1a-eGFP gonads and of cyp19a1a mRNA within the wild type gonads is reported herein. The rationale behind this discrepancy is not known at present and deserves further study.

In cyp19a1a-eGFP female ovaries, GFP was mainly localized to the cytoplasm of peri-follicular cells which is consistent with the localization of cyp19a1a mRNA in wild type zebrafish ovaries (Rodriguez-Mari et al., 2005; Wang and Orban, 2007). In ovaries, GFP fluorescence was higher than in immature gonads and increased during ovarian development in relation with the key role of 
cyp19a1a in ovarian differentiation in fish (Guiguen et al., 2010). In gonads of cyp19a1a-eGFP fish that undergo ovary-to-testis transition, GFP fluorescence levels were intermediate between females and males or similar to males. This pattern of gender expression of GFP is similar to the pattern of cyp19a1a gene expression in wild type zebrafish trunks/gonads (Rodriguez-Mari et al., 2005; Wang and Orban, 2007). The results of the present study suggest that cyp19a1a expression is necessary for the maintenance of the ovarian fate and that the down-regulation of cyp19a1a expression is likely responsible for testicular differentiation. Estrogens depletion may be one of the reasons leading to the degeneration of ovary during gonadal transformation in males as previously suggested by Wang and collaborators (2007). Recent studies in zebrafish but also tilapia and common carp showed that exposure of adult fish to aromatase inhibitors could lead to the transdifferentiation of fully differentiated ovaries into testis (Ogawa et al., 2008; Sun et al., 2014; Takatsu et al., 2013). Moreover, in tilapia, this transdifferentiation could be rescued by exogenous estradiol supplementation (Sun et al., 2014), confirming the importance of estrogens in ovarian maintenance.

Estrogens in fish are produced by two different aromatase proteins, i.e. Cyp19a1a and Cyp19a1b. While some results suggested a bimodal expression of cyp19a1b gene in the entire zebrafish larvae during the sexual differentiation process (Trant et al., 2001), more detailed studies established that cyp19a1b gene and protein are not expressed in a sexually dimorphic manner in the zebrafish larvae heads/brains during gonadal differentiation (Kallivretaki et al., 2007; Vosges et al., 2010). Moreover, a recent study showed no defects in the sexual differentiation process of cyp19a1b loss-of-function mutant zebrafish while cyp19a1a loss-of-function mutant zebrafish all developed as males (Dranow et al., 2016). Current studies thus do not support a decisive role of Cyp19a1b in the phenotypic sex orientation of the zebrafish gonads. Altogether, these observations rather support earlier studies (Fenske and Segner, 2004; McAllister and Kime, 2003; Uchida et al., 2004; Wang and Orban, 2007) that favor the hypothesis of Cyp19a1a being the rate-limiting step in female sexual development.

Regarding males, GFP expression was detectable in the present study in male gonads of the cyp19a1a-eGFP transgenic fish and this persisted during male development contrary to the absence of mRNA detection by in situ hybridization (Rodriguez-Mari et al., 2005). The presence of GFP in testis is relevant regarding the known cyp19a1a gene expression in male gonads both at the adult stage (Hinfray et al., 2006a; Sawyer et al., 2006) and during development (Wang and Orban, 2007). Estrogen receptors have also been localized in the testis of many fish including zebrafish (Bouma and Nagler, 2001; Chandrasekar et al., 2010; Menuet et al., 2002; Vinas and Piferrer, 2008; Wu et al., 2001). Altogether, these data suggest a role of estrogens in testis development, steroid production and spermatogenesis. Indeed, evidences indicate that spermatogonial stem cell renewal is regulated by estradiol (Miura et al., 1999; Miura et al., 2003).

\section{3. cyp19a1a-eGFP transgenic zebrafish as a reliable model to investigate reproductive functions and their perturbations by EDCs}


During the recent years, an increasing interest has emerged as regards in vivo transgenic fish models to assess environmental hazard of chemicals notably EDCs. Several transgenic fish models have been developed to study the effects of chemicals acting on the endocrine system through nuclear receptor pathways such as estrogen receptors (Gorelick and Halpern, 2011; Green et al., 2016; Legler et al., 2002) or glucocorticoid receptor (Weger et al., 2013) and disrupting the tissuespecific expression of hormone-regulated genes in the brain (Brion et al., 2012) or in the liver (Kurauchi et al., 2005; Sébillot et al., 2014; Spirhanzlova et al., 2016). Mechanism-based in vivo fish bioassays that use transgenic fish were recently developed and have been shown to be very useful tools to screen EDCs alone or in combination (Brion et al., 2012; Cano-Nicolau et al., 2016; Gorelick and Halpern, 2011; Green et al., 2016; Hinfray et al., 2016), some of them being under validation at the OECD level (i.e. EASZY assay that uses cyp19a1b-GFP zebrafish embryos). The usefulness of the newly developed cyp19a1a-eGFP line for studies on environmental effects of EDCs need to be further investigated. However, we demonstrated that this model has favorable and interesting features such as being a stable transgenic line, possessing only one transgene insertion with the possibility of inheritance of the transgene from both males and females making this line easier to maintain. Further, in adult cyp19a1a-eGFP transgenic zebrafish ovaries, pharmacological treatment with E2 resulted in inhibition of GFP fluorescence in a similar manner to cyp19a1a gene expression in wild type zebrafish ovaries (Hinfray et al., 2006a). Furthermore, the clear involvement of "ovarian" aromatase in the processes of reproduction and sexual differentiation in fish makes this transgenic zebrafish line a powerful model for studies onto these physiological processes and their perturbations by EDCs. Currently, at OECD, several test guidelines for screening and testing of endocrine active substances have been adapted in fish to detect compounds acting as aromatase inhibitors (Test Guideline 229/230) and affecting the sex development of the gonads and/or the sex-ratio (Test Guideline 234). The use of cyp19a1a-eGFP line for these assays should allow the relative quantification of GFP in gonads through image analysis thus providing additional mechanistic insight on aromatase that are currently lacking in OECD test guidelines. To do so, it would however be advisable to (i) evaluate the sensitivity of the cyp19a1a-eGFP zebrafish line to estrogens and also to other EDCs such as aromatase modulating compounds to refine actual mechanistic based assays, and (ii) improve the in vivo measurement of GFP in developing and adult fish in a non-invasive way to assess the timedependent effect of chemicals on GFP without increasing the number of fish. This could be notably achieved by cross-breeding our transgenic fish with the casper mutant zebrafish that do not develop pigments (White et al., 2008). Altogether, the time- and concentration-dependent measurement of GFP in live transgenic cyp19a1a-eGFP together with other "classical" endpoints (e.g., vitellogenin concentrations, gonadal histology) would usefully bring complementary comprehensive data for the refinement of Adverse Outcome Pathways (AOPs) and the development of quantitative AOPs thereby increasing the relevance of such model and bioassays for advanced assessment of EDCs in fish. 


\section{Conclusions}

In the present study, we successfully established a stable transgenic zebrafish line expressing GFP under the control of zebrafish cyp19a1a gene promoter. In the cyp19a1a-eGFP zebrafish line, GFP is expressed, localized and down-regulated by estradiol in the same way as cyp19a1a gene in wild type zebrafish showing that GFP can be used reliably to monitor cyp19a1a expression. In the cyp19a1a-eGFP transgenic line, our results confirm that cyp19a1a is expressed early during development, before the histological differentiation of the gonads, and that the down-regulation of cyp19a1a expression is likely responsible for testicular differentiation. Moreover, we show that although cyp19a1a expression exhibits a clear dimorphic expression pattern in gonads during sexual differentiation, its expression persists whatever the sex suggesting that estradiol synthesis is important for gonadal development including for testis where the expression was low but significant. In the end, monitoring the expression of GFP in control and exposed-fish will help determine the sensitivity of this transgenic line to EDCs and its relevance to refine mechanistic based-assays for the study of EDCs. In fine, this transgenic zebrafish line is a useful tool to study physiological processes such as reproduction and sexual differentiation, and their potential perturbations by EDCs.

\section{Declaration of interests}

The authors declare that there is no conflict of interest that could be perceived as prejudicing the impartiality of the research reported.

\section{Acknowledgements}

This work was supported by the French National Ministry of Ecology and Sustainable Development [Program 190 ECOT AP 2013-2016; NEMO project and Program 181-DRC 50] and by the French National Research Program for Environmental and Occupational Health of Anses [AIDEZ project $n^{\circ}$ EST-2016/1/189]. AMAGEN received the support of the French National Research Agency (ANR) "Investissement d'Avenir" program [TEFOR ANR-II-INSB-0014]. The authors wish to acknowledge the technical support of: S. Bruneau and A. Heuzé for the design and production of transgenesis vectors; Z. Radev and J. Edouard for the promoter cloning and transgenic generation; M. Simion and L. Legendre for zebrafish husbandry.

\section{References}

Baumann, L., Knorr, S., Keiter, S., Nagel, T., Rehberger, K., Volz, S., Oberrauch, S., Schiller, V., Fenske, M., Holbech, H., Segner, H., Braunbeck, T., 2014a. Persistence of endocrine disruption in zebrafish (Danio rerio) after discontinued exposure to the androgen 17beta-trenbolone. Environ. Toxicol. Chem. 33, 2488-2496. 
Baumann, L., Knorr, S., Keiter, S., Rehberger, K., Volz, S., Schiller, V., Fenske, M., Holbech, H., Segner, H., Braunbeck, T., 2014b. Reversibility of endocrine disruption in zebrafish (Danio rerio) after discontinued exposure to the estrogen 17alpha-ethinylestradiol. Toxicol. Appl. Pharmacol. 278, 230237.

Blazquez, M., Piferrer, F., 2004. Cloning, sequence analysis, tissue distribution, and sex-specific expression of the neural form of P450 aromatase in juvenile sea bass (Dicentrarchus labrax). Mol. Cell. Endocrinol. 219, 83-94.

Bouma, J., Nagler, J.J., 2001. Estrogen receptor-alpha protein localization in the testis of the rainbow trout (Oncorhynchus mykiss) during different stages of the reproductive cycle. Biol. Reprod. 65, 6065 .

Brion, F., Le Page, Y., Piccini, B., Cardoso, O., Tong, S.K., Chung, B.C., Kah, O., 2012. Screening estrogenic activities of chemicals or mixtures in vivo using transgenic (cyp19a1b-GFP) zebrafish embryos. PLoS One 7, e36069.

Brion, F., Tyler, C.R., Palazzi, X., Laillet, B., Porcher, J.M., Garric, J., Flammarion, P., 2004. Impacts of 17[beta]-estradiol, including environmentally relevant concentrations, on reproduction after exposure during embryo-larval-, juvenile- and adult-life stages in zebrafish (Danio rerio). Aquat. Toxicol. 68, 193-217.

Cano-Nicolau, J., Garoche, C., Hinfray, N., Pellegrini, E., Boujrad, N., Pakdel, F., Kah, O., Brion, F., 2016. Several synthetic progestins disrupt the glial cell specific-brain aromatase expression in developing zebrafish. Toxicol. Appl. Pharmacol. 305, 12-21.

Caulier, M., Brion, F., Chadili, E., Turies, C., Piccini, B., Porcher, J.-M., Guiguen, Y., Hinfray, N., 2015. Localization of steroidogenic enzymes and Foxl2a in the gonads of mature zebrafish (Danio rerio). Comp. Biochem. Physiol., Part A Mol. Integr. Physiol. 188, 96-106.

Chandrasekar, G., Archer, A., Gustafsson, J.A., Andersson Lendahl, M., 2010. Levels of 17betaestradiol receptors expressed in embryonic and adult zebrafish following in vivo treatment of natural or synthetic ligands. PLoS One 5, e9678.

Chang, X., Kobayashi, T., Senthilkumaran, B., Kobayashi-Kajura, H., Sudhakumari, C.C., Nagahama, Y., 2005. Two types of aromatase with different encoding genes, tissue distribution and developmental expression in Nile tilapia (Oreochromis niloticus). Gen. Comp. Endocrinol. 141, 101-115.

Chiang, E.F., Yan, Y.L., Guiguen, Y., Postlethwait, J., Chung, B., 2001a. Two Cyp19 (P450 aromatase) genes on duplicated zebrafish chromosomes are expressed in ovary or brain. Mol. Biol. Evol. 18, 542-550.

Chiang, E.F., Yan, Y.L., Tong, S.K., Hsiao, P.H., Guiguen, Y., Postlethwait, J., Chung, B.C., 2001b. Characterization of duplicated zebrafish cyp19 genes. J. Exp. Zool. 290, 709-714.

Dalla Valle, L., Lunardi, L., Colombo, L., Belvedere, P., 2002a. European sea bass (Dicentrarchus labrax L.) cytochrome P450arom: cDNA cloning, expression and genomic organization. J. Steroid Biochem. Mol. Biol. 80, 25-34.

Dalla Valle, L., Ramina, A., Vianello, S., Belvedere, P., Colombo, L., 2002b. Cloning of two mRNA variants of brain aromatase cytochrome P450 in rainbow trout (Oncorhynchus mykiss Walbaum). J. Steroid Biochem. Mol. Biol. 82, 19-32.

Deng, J., Liu, C., Yu, L., Zhou, B., 2010. Chronic exposure to environmental levels of tribromophenol impairs zebrafish reproduction. Toxicol. Appl. Pharmacol. 243, 87-95. 
Dranow, D.B., Hu, K., Bird, A.M., Lawry, S.T., Adams, M.T., Sanchez, A., Amatruda, J.F., Draper, B.W., 2016. Bmp15 Is an Oocyte-Produced Signal Required for Maintenance of the Adult Female Sexual Phenotype in Zebrafish. PLoS Genetics 12, e1006323.

Emelyanov, A., Parinov, S., 2008. Mifepristone-inducible LexPR system to drive and control gene expression in transgenic zebrafish. Dev. Biol. 320, 113-121.

Fenske, M., Segner, H., 2004. Aromatase modulation alters gonadal differentiation in developing zebrafish (Danio rerio). Aquat. Toxicol. 67, 105-126.

Gohin, M., Bodinier, P., Fostier, A., Chesnel, F., Bobe, J., 2011. Aromatase is expressed and active in the rainbow trout oocyte during final oocyte maturation. Mol. Reprod. Dev. 78, 510-518.

Gorelick, D.A., Halpern, M.E., 2011. Visualization of estrogen receptor transcriptional activation in zebrafish. Endocrinol. 152, 2690-2703.

Goto-Kazeto, R., Kight, K.E., Zohar, Y., Place, A.R., Trant, J.M., 2004. Localization and expression of aromatase mRNA in adult zebrafish. Gen. Comp. Endocrinol. 139, 72-84.

Green, J.M., Metz, J., Lee, O., Trznadel, M., Takesono, A., Brown, A.R., Owen, S.F., Kudoh, T., Tyler, C.R., 2016. High-Content and Semi-Automated Quantification of Responses to Estrogenic Chemicals Using a Novel Translucent Transgenic Zebrafish. Environ. Sci. Technol. 50, 6536-6545.

Guiguen, Y., Fostier, A., Piferrer, F., Chang, C.-F., 2010. Ovarian aromatase and estrogens: A pivotal role for gonadal sex differentiation and sex change in fish. Gen. Comp. Endocrinol. 165, 352-366.

Hinfray, N., Nobrega, R.H., Caulier, M., Baudiffier, D., Maillot-Maréchal, E., Chadili, E., Palluel, O., Porcher, J.-M., Schulz, R., Brion, F., 2013. Cyp17a1 and Cyp19a1 in the zebrafish testis are differentially affected by oestradiol. J. Endocrinol. 216, 375-388.

Hinfray, N., Palluel, O., Turies, C., Cousin, C., Porcher, J.M., Brion, F., 2006a. Brain and gonadal aromatase as potential targets of endocrine disrupting chemicals in a model species, the zebrafish (Danio rerio). Environ. Toxicol. 21, 332-337.

Hinfray, N., Porcher, J.M., Brion, F., 2006b. Inhibition of rainbow trout (Oncorhynchus mykiss) P450 aromatase activities in brain and ovarian microsomes by various environmental substances. Comp. Biochem. Physiol. C Toxicol. Pharmacol. 144, 252-262.

Hinfray, N., Tebby, C., Garoche, C., Piccini, B., Bourgine, G., Ait-Aissa, S., Kah, O., Pakdel, F., Brion, F., 2016. Additive effects of levonorgestrel and ethinylestradiol on brain aromatase (cyp19a1b) in zebrafish specific in vitro and in vivo bioassays. Toxicol. Appl. Pharmacol. 307, 108-114.

Holbech, H., Kinnberg, K., Petersen, G.I., Jackson, P., Hylland, K., Norrgren, L., Bjerregaard, P., 2006. Detection of endocrine disrupters: Evaluation of a Fish Sexual Development Test (FSDT). Comp. Biochem. Physiol. C Toxicol. Pharmacol. 144, 57-66.

Ji, K., Hong, S., Kho, Y., Choi, K., 2013. Effects of Bisphenol S Exposure on Endocrine Functions and Reproduction of Zebrafish. Environ. Sci. Technol. 47, 8793-8800.

Kallivretaki, E., Eggen, R.I., Neuhauss, S.C., Kah, O., Segner, H., 2007. The zebrafish, brain-specific, aromatase cyp19a2 is neither expressed nor distributed in a sexually dimorphic manner during sexual differentiation. Dev. Dyn. 236, 3155-3166. 
Kitano, T., Takamune, K., Nagahama, Y., Abe, S.I., 2000. Aromatase inhibitor and 17alphamethyltestosterone cause sex-reversal from genetical females to phenotypic males and suppression of P450 aromatase gene expression in Japanese flounder (Paralichthys olivaceus). Mol. Reprod. Dev. 56, $1-5$.

Klein, C., Mikutta, J., Krueger, J., Scholz, K., Brinkmann, J., Liu, D., Veerkamp, J., Siegel, D., Abdelilah-Seyfried, S., le Noble, F., 2011. Neuron navigator 3a regulates liver organogenesis during zebrafish embryogenesis. Development 138, 1935-1945.

Kobayashi, Y., Kobayashi, T., Nakamura, M., Sunobe, T., Morrey, C.E., Suzuki, N., Nagahama, Y., 2004. Characterization of two types of cytochrome P450 aromatase in the serial-sex changing gobiid fish, Trimma okinawae. Zoolog. Sci. 21, 417-425.

Kotula-Balak, M., Zielinska, R., Glogowski, J., Kowalski, R.K., Sarosiek, B., Bilinska, B., 2008. Aromatase expression in testes of $\mathrm{XY}, \mathrm{YY}$, and $\mathrm{XX}$ rainbow trout (Oncorhynchus mykiss). Comp. Biochem. Physiol., Part A Mol. Integr. Physiol. 149, 188-196.

Kurauchi, K., Nakaguchi, Y., Tsutsumi, M., Hori, H., Kurihara, R., Hashimoto, S., Ohnuma, R., Yamamoto, Y., Matsuoka, S., Kawai, S., Hirata, T., Kinoshita, M., 2005. In vivo visual reporter system for detection of estrogen-like substances by transgenic medaka. Environ. Sci. Technol. 39, $2762-2768$.

Legler, J., Zeinstra, L.M., Schuitemaker, F., Lanser, P.H., Bogerd, J., Brouwer, A., Vethaak, A.D., De Voogt, P., Murk, A.J., Van der Burg, B., 2002. Comparison of in vivo and in vitro reporter gene assays for short-term screening of estrogenic activity. Environ. Sci. Technol. 36, 4410-4415.

Liew, W.C., Orban, L., 2014. Zebrafish sex: a complicated affair. Brief. Funct. Genomics 13, 172-187.

Liu, X., Ji, K., AreumJo, Moon, H.-B., Choi, K., 2013. Effects of TDCPP or TPP on gene transcriptions and hormones of HPG axis, and their consequences on reproduction in adult zebrafish (Danio rerio). Aquat. Toxicol. 134-135, 104-111.

Liu, X., Ji, K., Choi, K., 2012. Endocrine disruption potentials of organophosphate flame retardants and related mechanisms in H295R and MVLN cell lines and in zebrafish. Aquat. Toxicol. 114-115, 173-181.

Luzio, A., Coimbra, A.M., Benito, C., Fontainhas-Fernandes, A.A., Matos, M., 2015. Screening and identification of potential sex-associated sequences in Danio rerio. Mol. Reprod. Dev. 82, 756-764.

Ma, Y., Han, J., Guo, Y., Lam, P.K.S., Wu, R.S.S., Giesy, J.P., Zhang, X., Zhou, B., 2012. Disruption of endocrine function in in vitro H295R cell-based and in in vivo assay in zebrafish by 2,4dichlorophenol. Aquat. Toxicol. 106-107, 173-181.

Maack, G., Segner, H., 2003. Morphological development of the gonads in zebrafish. J. Fish Biol. 62, 895-906.

Maack, G., Segner, H., 2004. Life-stage-dependent sensitivity of zebrafish (Danio rerio) to estrogen exposure. Comp. Biochem. Physiol. C Toxicol. Pharmacol. 139, 47-55.

McAllister, B.G., Kime, D.E., 2003. Early life exposure to environmental levels of the aromatase inhibitor tributyltin causes masculinisation and irreversible sperm damage in zebrafish (Danio rerio). Aquat. Toxicol. 65, 309-316. 
Menuet, A., Pellegrini, E., Anglade, I., Blaise, O., Laudet, V., Kah, O., Pakdel, F., 2002. Molecular characterization of three estrogen receptor forms in zebrafish: binding characteristics, transactivation properties, and tissue distributions. Biol. Reprod. 66, 1881-1892.

Menuet, A., Pellegrini, E., Brion, F., Gueguen, M.M., Anglade, I., Pakdel, F., Kah, O., 2005. Expression and estrogen-dependent regulation of the zebrafish brain aromatase gene. J. Comp. Neurol. $485,304-320$.

Miura, T., Miura, C., Ohta, T., Nader, M.R., Todo, T., Yamauchi, K., 1999. Estradiol-17[beta] Stimulates the Renewal of Spermatogonial Stem Cells in Males. Biochem. Biophys. Res. Commun. 264, 230-234.

Miura, T., Ohta, T., Miura, C.I., Yamauchi, K., 2003. Complementary deoxyribonucleic acid cloning of spermatogonial stem cell renewal factor. Endocrinol. 144, 5504-5510.

Morthorst, J.E., Holbech, H., Bjerregaard, P., 2010. Trenbolone causes irreversible masculinization of zebrafish at environmentally relevant concentrations. Aquat. Toxicol. 98, 336-343.

Nagabhushana, A., Mishra, R.K., 2016. Finding clues to the riddle of sex determination in zebrafish. J. biosci. 41, 145-155.

Nagahama, Y., 1994. Endocrine regulation of gametogenesis in fish. Int. J. Dev. Biol. 38, 217-229.

Ogawa, S., Akiyoshi, M., Higuchi, M., Nakamura, M., Hirai, T., 2008. 'Post-sex differentiational' sex reversal in the feamle common carp (Cyprinus carpio). Cybium 32, 102-103.

Park, J.W., Tompsett, A., Zhang, X., Newsted, J.L., Jones, P.D., Au, D., Kong, R., Wu, R.S., Giesy, J.P., Hecker, M., 2008. Fluorescence in situ hybridization techniques (FISH) to detect changes in CYP19a gene expression of Japanese medaka (Oryzias latipes). Toxicol. Appl. Pharmacol. 232, 226235.

Pellegrini, E., Mouriec, K., Anglade, I., Menuet, A., Le Page, Y., Gueguen, M.M., Marmignon, M.H., Brion, F., Pakdel, F., Kah, O., 2007. Identification of aromatase-positive radial glial cells as progenitor cells in the ventricular layer of the forebrain in zebrafish. J. Comp. Neurol. 501, 150-167.

Raghuveer, K., Senthilkumaran, B., Sudhakumari, C.C., Sridevi, P., Rajakumar, A., Singh, R., Murugananthkumar, R., Majumdar, K.C., 2011. Dimorphic expression of various transcription factor and steroidogenic enzyme genes during gonadal ontogeny in the air-breathing catfish, Clarias gariepinus. Sex. Dev. 5, 213-223.

Rodriguez-Mari, A., Yan, Y.-L., BreMiller, R.A., Wilson, C., Canestro, C., Postlethwait, J.H., 2005. Characterization and expression pattern of zebrafish anti-Mullerian hormone (amh) relative to sox9a, sox9b, and cyp19a1a, during gonad development. Gene Expr. Patterns 5, 655-667.

Roure, A., Rothbächer, U., Robin, F., Kalmar, E., Ferone, G., Lamy, C., Missero, C., Mueller, F., Lemaire, P., 2007. A Multicassette Gateway Vector Set for High Throughput and Comparative Analyses in Ciona and Vertebrate Embryos. PLoS One 2, e916.

Sanderson, J.T., Boerma, J., Lansbergen, G.W.A., van den Berg, M., 2002. Induction and Inhibition of Aromatase (CYP19) Activity by Various Classes of Pesticides in H295R Human Adrenocortical Carcinoma Cells. Toxicol. Appl. Pharmacol. 182, 44-54.

Sawyer, S.J., Gerstner, K.A., Callard, G.V., 2006. Real-time PCR analysis of cytochrome P450 aromatase expression in zebrafish: Gene specific tissue distribution, sex differences, developmental programming, and estrogen regulation. Gen. Comp. Endocrinol. 142, 108-117. 
Sébillot, A., Damdimopoulou, P., Ogino, Y., Spirhanzlova, P., Miyagawa, S., Du Pasquier, D., Mouatassim, N., Iguchi, T., Lemkine, G.F., Demeneix, B.A., Tindall, A.J., 2014. Rapid Fluorescent Detection of (Anti)androgens with spiggin-gfp Medaka. Environ. Sci. Technol. 48, 10919-10928.

Skolness, S.Y., Blanksma, C.A., Cavallin, J.E., Churchill, J.J., Durhan, E.J., Jensen, K.M., Johnson, R.D., Kahl, M.D., Makynen, E.A., Villeneuve, D.L., Ankley, G.T., 2013. Propiconazole Inhibits Steroidogenesis and Reproduction in the Fathead Minnow (Pimephales promelas). Toxicol. Sci. 132, 284-297.

Spirhanzlova, P., Leleu, M., Sébillot, A., Lemkine, G.F., Iguchi, T., Demeneix, B.A., Tindall, A.J., 2016. Oestrogen reporter transgenic medaka for non-invasive evaluation of aromatase activity. Comp. Biochem. Physiol. C Toxicol. Pharmacol. 179, 64-71.

Strykowski, J.L., Schech, J.M., 2015. Effectiveness of recommended euthanasia methods in larval zebrafish (Danio rerio). J. Am. Assoc. Lab. Anim. Sci. 54, 81-84.

Sun, L., Zha, J., Spear, P.A., Wang, Z., 2007. Toxicity of the aromatase inhibitor letrozole to Japanese medaka (Oryzias latipes) eggs, larvae and breeding adults. Comp. Biochem. Physiol. C Toxicol. Pharmacol. 145, 533-541.

Sun, L.N., Jiang, X.L., Xie, Q.P., Yuan, J., Huang, B.F., Tao, W.J., Zhou, L.Y., Nagahama, Y., Wang, D.S., 2014. Transdifferentiation of differentiated ovary into functional testis by long-term treatment of aromatase inhibitor in Nile tilapia. Endocrinol. 155, 1476-1488.

Takahashi, H., 1977. Juvenile hermaphroditism in the Zebrafish, Brachydanio rerio. Bull. Fac. Fish. Hokkaido Univ. 28, 57-65.

Takatsu, K., Miyaoku, K., Roy, S.R., Murono, Y., Sago, T., Itagaki, H., Nakamura, M., Tokumoto, T., 2013. Induction of Female-to-Male Sex Change in Adult Zebrafish by Aromatase Inhibitor Treatment. Sci. Rep. 3.

Tchoudakova, A., Callard, G.V., 1998. Identification of multiple CYP19 genes encoding different cytochrome P450 aromatase isozymes in brain and ovary. Endocrinol. 139, 2179-2189.

Thermes, V., Grabher, C., Ristoratore, F., Bourrat, F., Choulika, A., Wittbrodt, J., Joly, J.-S., 2002. ISceI meganuclease mediates highly efficient transgenesis in fish. Mech. Dev. 118, 91-98.

Trant, J.M., Gavasso, S., Ackers, J., Chung, B.C., Place, A.R., 2001. Developmental expression of cytochrome P450 aromatase genes (CYP19a and CYP19b) in zebrafish fry (Danio rerio). J. Exp. Zool. 290, 475-483.

Uchida, D., Yamashita, M., Kitano, T., Iguchi, T., 2004. An aromatase inhibitor or high water temperature induce oocyte apoptosis and depletion of $\mathrm{P} 450$ aromatase activity in the gonads of genetic female zebrafish during sex-reversal. Comp. Biochem. Physiol., Part A Mol. Integr. Physiol. 137, 1120.

Vinas, J., Piferrer, F., 2008. Stage-specific gene expression during fish spermatogenesis as determined by laser-capture microdissection and quantitative-PCR in sea bass (Dicentrarchus labrax) gonads. Biol. Reprod. 79, 738-747.

von Schalburg, K.R., Gowen, B.E., Rondeau, E.B., Johnson, N.W., Minkley, D.R., Leong, J.S., Davidson, W.S., Koop, B.F., 2013. Sex-specific expression, synthesis and localization of aromatase regulators in one-year-old Atlantic salmon ovaries and testes. Comp. Biochem. Physiol. B, Biochem. Mol. Biol. 164, 236-246. 
Vosges, M., Le Page, Y., Chung, B.C., Combarnous, Y., Porcher, J.M., Kah, O., Brion, F., 2010. 17alpha-ethinylestradiol disrupts the ontogeny of the forebrain GnRH system and the expression of brain aromatase during early development of zebrafish. Aquat. Toxicol. 99, 479-491.

Wang, X.G., Orban, L., 2007. Anti-Mullerian hormone and 11 beta-hydroxylase show reciprocal expression to that of aromatase in the transforming gonad of zebrafish males. Dev. Dyn. 236, 13291338.

Weger, B.D., Weger, M., Jung, N., Lederer, C., Brase, S., Dickmeis, T., 2013. A chemical screening procedure for glucocorticoid signaling with a zebrafish larva luciferase reporter system. J. Vis. Exp. 79: 50439.

White, R.M., Sessa, A., Burke, C., Bowman, T., LeBlanc, J., Ceol, C., Bourque, C., Dovey, M., Goessling, W., Burns, C.E., Zon, L.I., 2008. Transparent adult zebrafish as a tool for in vivo transplantation analysis. Cell stem cell 2, 183-189.

Wilson, C.A., High, S.K., McCluskey, B.M., Amores, A., Yan, Y.L., Titus, T.A., Anderson, J.L., Batzel, P., Carvan, M.J., 3rd, Schartl, M., Postlethwait, J.H., 2014. Wild sex in zebrafish: loss of the natural sex determinant in domesticated strains. Genetics 198, 1291-1308.

Wilson, J.M., Bunte, R.M., Carty, A.J. 2009. Evaluation of rapid cooling and tricaine methanesulfonate (MS222) as methods of euthanasia in zebrafish (Danio rerio). J. Am. Assoc. Lab. Anim. Sci. 48, 785-789.

Wu, C., Patino, R., Davis, K.B., Chang, X., 2001. Localization of estrogen receptor alpha and beta RNA in germinal and nongerminal epithelia of the channel catfish testis. Gen. Comp. Endocrinol. 124, $12-20$.

Yano, A., Nicol, B., Guerin, A., Guiguen, Y., 2011. The duplucated Rainbow Trout (Oncorhynchus mykiss) T-box transcription factors 1 , tbx $1 \mathrm{a}$ and tbx1b, are up-regulated during testicular development. Mol. Reprod. Dev. 78, 172-180.

Yu, L., Liu, C., Chen, Q., Zhou, B., 2014. Endocrine disruption and reproduction impairment in zebrafish after long-term exposure to DE-71. Environ. Toxicol. Chem. 33, 1354-1362.

Zapater, C., Chauvigne, F., Scott, A.P., Gomez, A., Katsiadaki, I., Cerda, J., 2012. Piscine folliclestimulating hormone triggers progestin production in gilthead seabream primary ovarian follicles. Biol. Reprod. 87, 111. 


\section{SUPPLEMENTARY MATERIALS}

Supplemental Table 1: Sequence of the primers used to clone and verify the promoter of Cyp19a1a. The underlined sequences in Cyp19a1a_B3 and Cyp19a1a_B5 primers correspond respectively to the attB3 and attB5 sites inserted during the second round of nested PCR. Those recombination sites are necessary for the cloning by Gateway $(R)$ recombination.

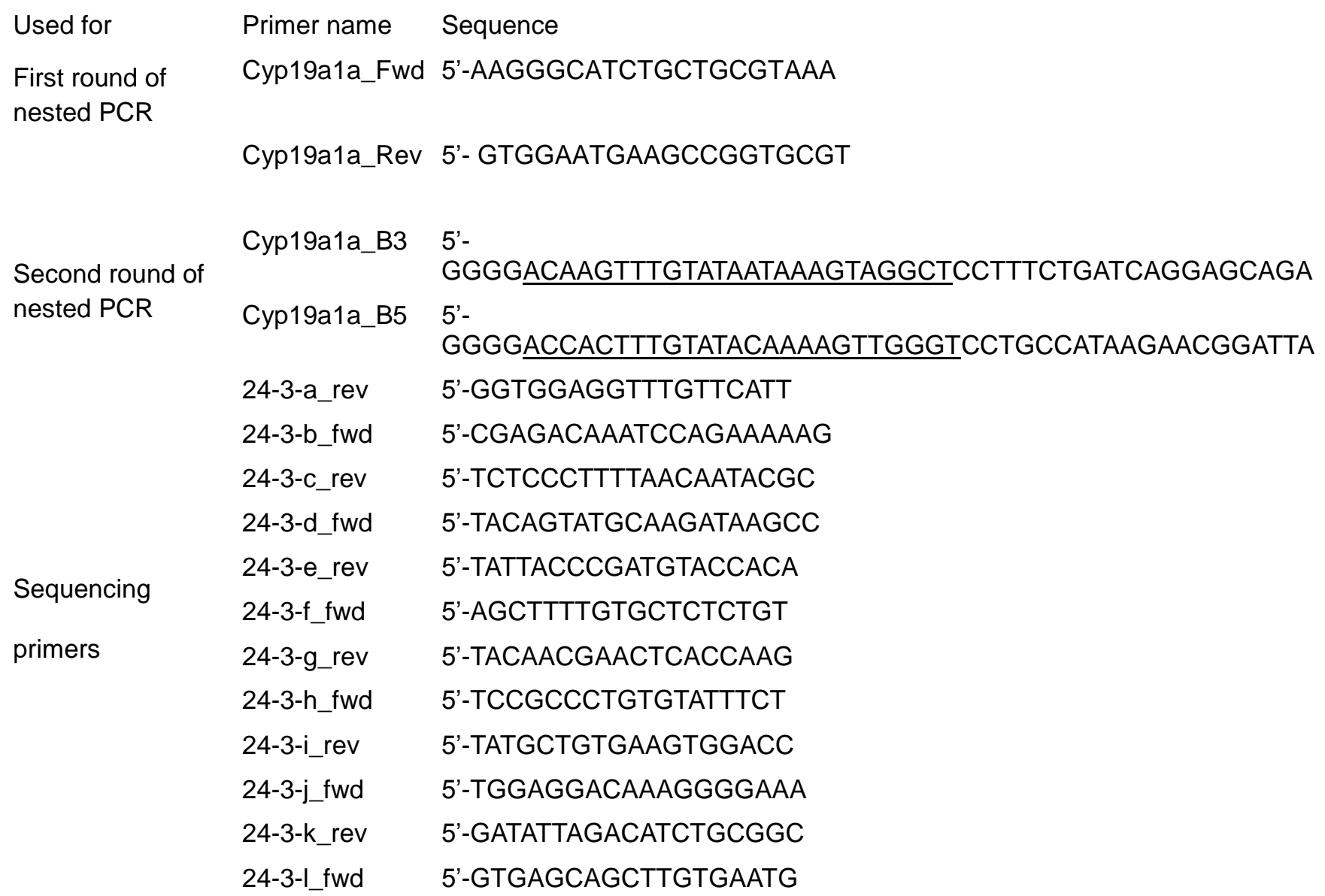


Supplemental figure 1 
$\begin{array}{ll}\text { Name } & \text { pDONR35H } \\ \text { Type } & \text { Donor Vector } \\ \text { Size } & 4762 \mathrm{bp} \\ \text { Origine } & \text { Roure et al, (2007) PLoS ONE 2(9): e916. }\end{array}$

Selectable makers Kanamycin - chloramphenicol

Bacterial strain DB3.1(TM) or any [gyrA462]

Comments listed as 'pDONR-221-P3/P5' in Roure et al.

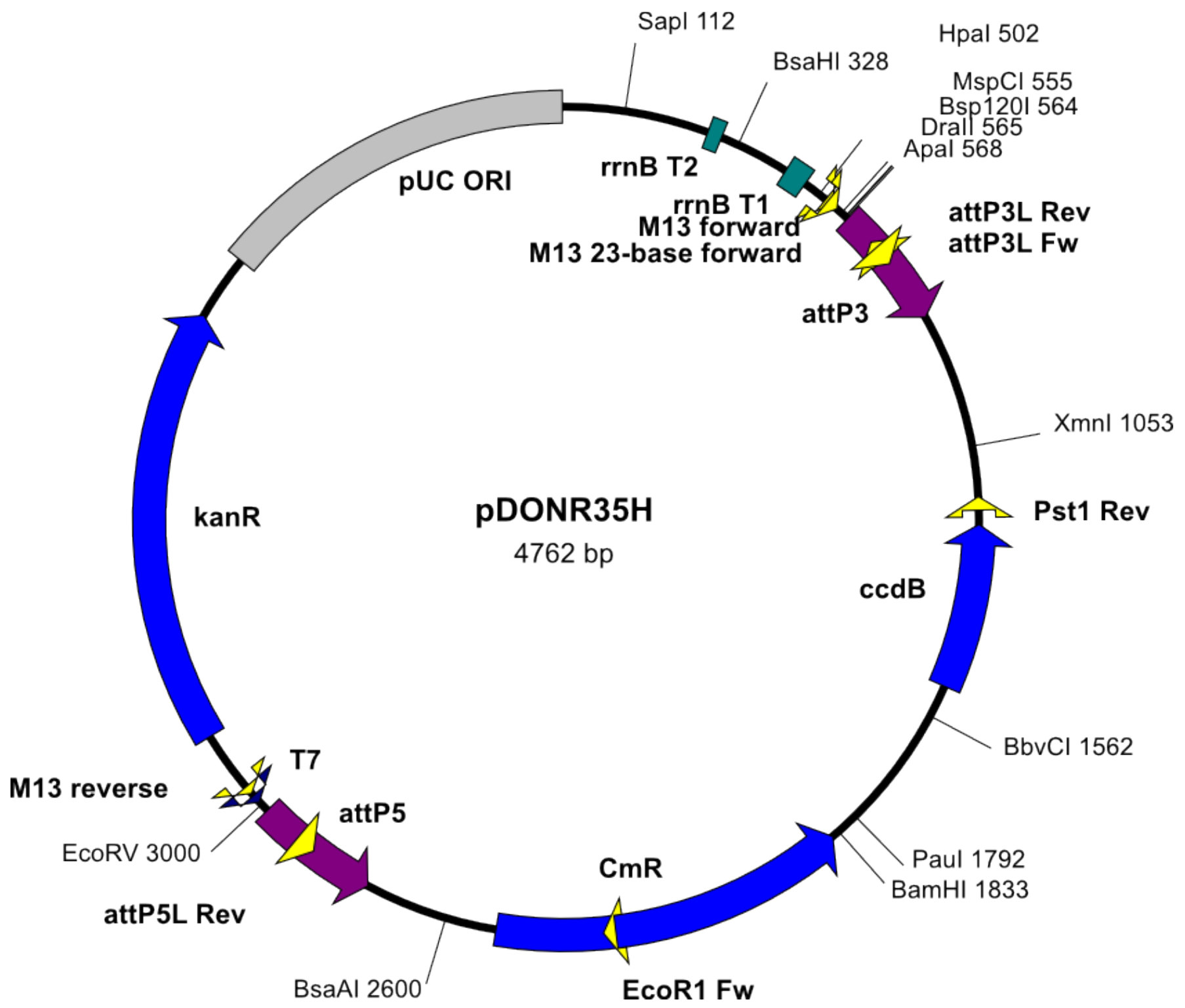


The elements found are detailed below

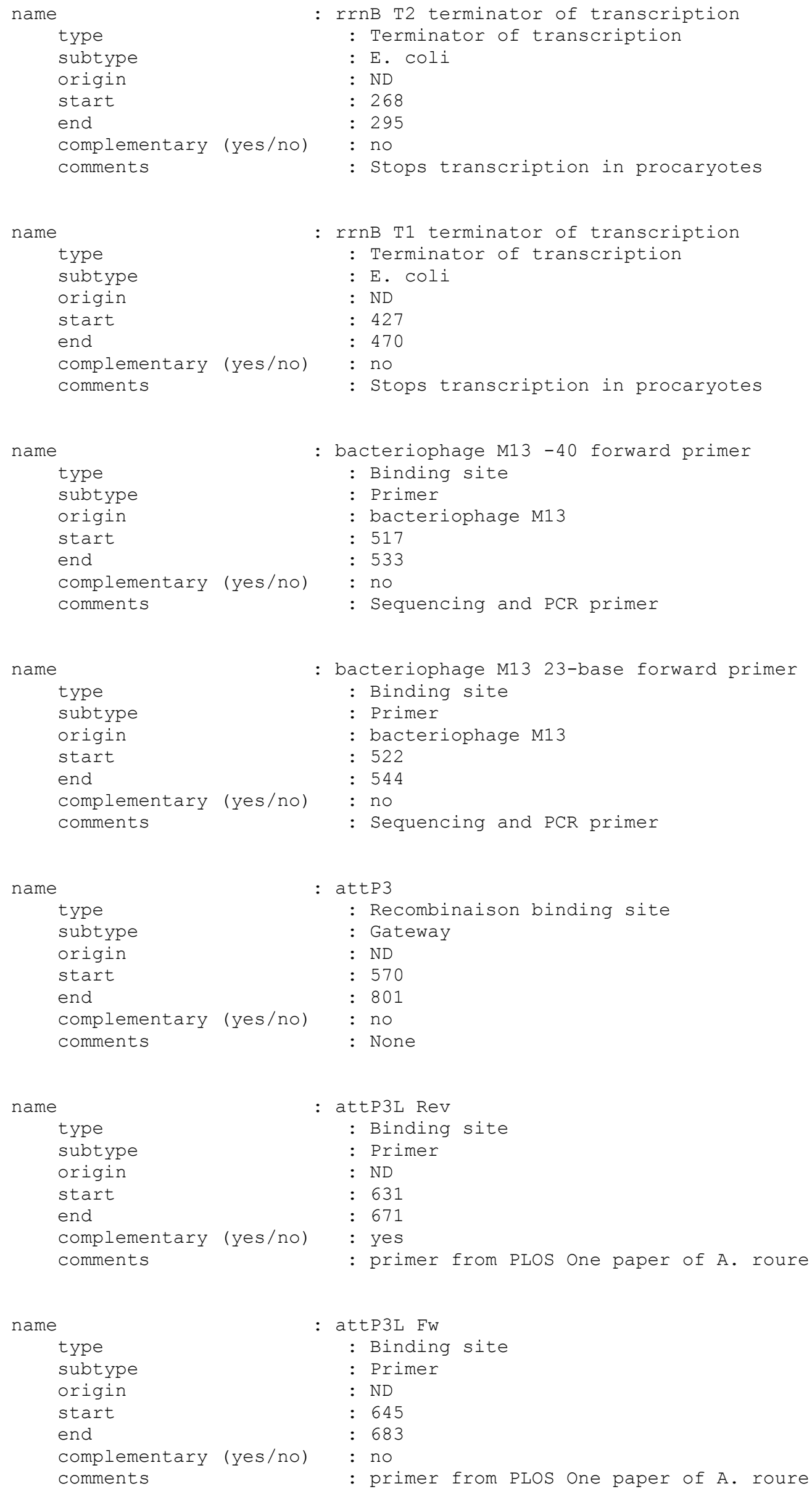









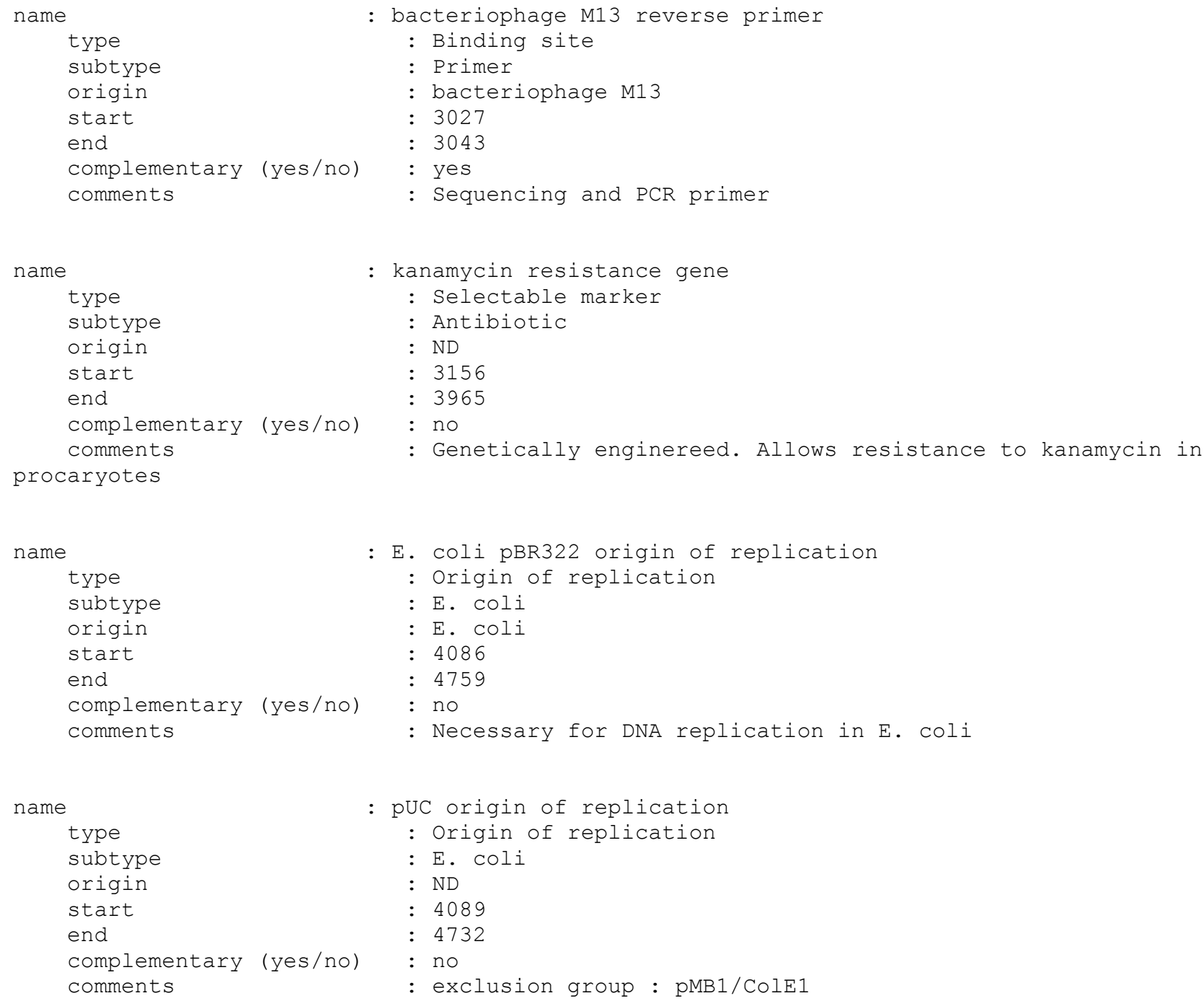


>pDONR35H - 4762 bp - circular - Thu Jan 21 08:43:43 2010 СTTTCCTGCGTTATCCCCTGATTCTGTGGATAACCGTATTACCGCCTTTGAGTGAGCTGA TACCGCTCGCCGCAGCCGAACGACCGAGCGCAGCGAGTCAGTGAGCGAGGAAGCGGAAGA GCGCCCAATACGCAAACCGCCTCTCCCCGCGCGTTGGCCGATTCATTAATGCAGCTGGCA CGACAGGTTTCCCGACTGGAAAGCGGGCAGTGAGCGCAACGCAATTAATACGCGTACCGC TAGCCAGGAAGAGTTTGTAGAAACGCAAAAAGGCCATCCGTCAGGATGGCCTTCTGCTTA GTTTGATGCCTGGCAGTTTATGGCGGGCGTCCTGCCCGCCACCCTCCGGGCCGTTGCTTC ACAACGTTCAAATCCGCTCCCGGCGGATTTGTCCTACTCAGGAGAGCGTTCACCGACAAA CAACAGATAAAACGAAAGGCCCAGTCTTCCGACTGAGCCTTTCGTTTTATTTGATGCCTG GCAGTTCCCTACTCTCGCGTTAACGCTAGCATGGATGTTTTCCCAGTCACGACGTTGTAA AACGACGGCCAGTCTTAAGCTCGGGCCCCAAATAATGATTTTATTTTGACTGATAGTGAC CTGTTCGTTGCAACACATTGATGAGCAATGCTTTTTTATAATGCCAACTTTGTATAATAA AGTTGAACGAGAAACGTAAAATGATATAAATATCAATATATTAAATTAGATTTTGCATAA AAAACAGACTACATAATACTGTAAAACACAACATATCCAGTCACTATGAATCAACTACTT AGATGGTATTAGTGACCTGTAGTCGACCGACAGCCTTCCAAATGTTCTTCGGGTGATGCT GCCAACTTAGTCGACCGACAGCCTTCCAAATGTTCTTCTCAAACGGAATCGTCGTATCCA GCCTACTCGCTATTGTCCTCAATGCCGTATTAAATCATAAAAAGAAATAAGAAAAAGAGG TGCGAGCCTCTTTTTTGTGTGACAAAATAAAAACATCTACCTATTCATATACGCTAGTGT CATAGTCCTGAAAATCATCTGCATCAAGAACAATTTCACAACTCTTATACTTTTCTCTTA CAAGTCGTTCGGCTTCATCTGGATTTTCAGCCTCTATACTTACTAAACGTGATAAAGTTT CTGTAATTTCTACTGTATCGACCTGCAGACTGGCTGTGTATAAGGGAGCCTGACATTTAT ATTCCCCAGAACATCAGGTTAATGGCGTTTTTGATGTCATTTTCGCGGTGGCTGAGATCA GCCACTTCTTCCCCGATAACGGAGACCGGCACACTGGCCATATCGGTGGTCATCATGCGC CAGCTTTCATCCCCGATATGCACCACCGGGTAAAGTTCACGGGAGACTTTATCTGACAGC AGACGTGCACTGGCCAGGGGGATCACCATCCGTCGCCCGGGCGTGTCAATAATATCACTC TGTACATCCACAAACAGACGATAACGGCTCTCTCTTTTATAGGTGTAAACCTTAAACTGC ATTTCACCAGCCCCTGTTCTCGTCAGCAAAAGAGCCGTTCATTTCAATAAACCGGGCGAC CTCAGCCATCCCTTCCTGATTTTCCGCTTTCCAGCGTTCGGCACGCAGACGACGGGCTTC ATTCTGCATGGTTGTGCTTACCAGACCGGAGATATTGACATCATATATGCCTTGAGCAAC TGATAGCTGTCGCTGTCAACTGTCACTGTAATACGCTGCTTCATAGCATACCTCTTTTTG ACATACTTCGGGTATACATATCAGTATATATTCTTATACCGCAAAAATCAGCGCGCAAAT ACGCATACTGTTATCTGGCTTTTAGTAAGCCGGATCCACGCGGCGTTTACGCCCCGCCCT GCCACTCATCGCAGTACTGTTGTAATTCATTAAGCATTCTGCCGACATGGAAGCCATCAC AGACGGCATGATGAACCTGAATCGCCAGCGGCATCAGCACCTTGTCGCCTTGCGTATAAT ATTTGCCCATGGTGAAAACGGGGGCGAAGAAGTTGTCCATATTGGCCACGTTTAAATCAA AACTGGTGAAACTCACCCAGGGATTGGCTGAGACGAAAAACATATTCTCAATAAACCCTT TAGGGAAATAGGCCAGGTTTTCACCGTAACACGCCACATCTTGCGAATATATGTGTAGAA ACTGCCGGAAATCGTCGTGGTATTCACTCCAGAGCGATGAAAACGTTTCAGTTTGCTCAT GGAAAACGGTGTAACAAGGGTGAACACTATCCCATATCACCAGCTCACCGTCTTTCATTG CCATACGGAATTCCGGATGAGCATTCATCAGGCGGGCAAGAATGTGAATAAAGGCCGGAT AAAACTTGTGCTTATTTTTCTTTACGGTCTTTAAAAAGGCCGTAATATCCAGCTGAACGG TCTGGTTATAGGTACATTGAGCAACTGACTGAAATGCCTCAAAATGTTCTTTACGATGCC ATTGGGATATATCAACGGTGGTATATCCAGTGATTTTTTTCTCCATTTTAGCTTCCTTAG СTCCTGAAAATCTCGATAACTCAAAAAATACGCCCGGTAGTGATCTTATTTCATTATGGT GAAAGTTGGAACCTCTTACGTGCCGATCAACGTCTCATTTTCGCCAAAAGTTGGCCCAGG GCTTCCCGGTATCAACAGGGACACCAGGATTTATTTATTCTGCGAAGTGATCTTCCGTCA CAGGTATTTATTCGGCGCAAAGTGCGTCGGGTGATGCTGCCAACTTAGTCGACTACAGGT CACTAATACCATCTAAGTAGTTGATTCATAGTGACTGGATATGTTGTGTTTTACAGTATT ATGTAGTCTGTTTTTTATGCAAAATCTAATTTAATATATTGATATTTATATCATTTTACG TTTCTCGTTCAACTTTTGTATACAAAGTTGGCATTATAAGAAAGCATTGCTTATCAATTT GTTGCAACGAACAGGTCACTATCAGTCAAAATAAAATCATTATTTGCCATCCAGCTGATA TCCCCTATAGTGAGTCGTATTACATGGTCATAGCTGTTTCCTGGCAGCTCTGGCCCGTGT СTCAAAATCTCTGATGTTACATTGCACAAGATAAAATAATATCATCATGAACAATAAAAC TGTCTGCTTACATAAACAGTAATACAAGGGGTGTTATGAGCCATATTCAACGGGAAACGT CGAGGCCGCGATTAAATTCCAACATGGATGCTGATTTATATGGGTATAAATGGGCTCGCG ATAATGTCGGGCAATCAGGTGCGACAATCTATCGCTTGTATGGGAAGCCCGATGCGCCAG AGTTGTTTCTGAAACATGGCAAAGGTAGCGTTGCCAATGATGTTACAGATGAGATGGTCA GACTAAACTGGCTGACGGAATTTATGCCTCTTCCGACCATCAAGCATTTTATCCGTACTC CTGATGATGCATGGTTACTCACCACTGCGATCCCCGGAAAAACAGCATTCCAGGTATTAG AAGAATATCCTGATTCAGGTGAAAATATTGTTGATGCGCTGGCAGTGTTCCTGCGCCGGT TGCATTCGATTCCTGTTTGTAATTGTCCTTTTAACAGCGATCGCGTATTTCGTCTCGCTC AGGCGCAATCACGAATGAATAACGGTTTGGTTGATGCGAGTGATTTTGATGACGAGCGTA ATGGCTGGCCTGTTGAACAAGTCTGGAAAGAAATGCATAAACTTTTGCCATTCTCACCGG ATTCAGTCGTCACTCATGGTGATTTCTCACTTGATAACCTTATTTTTGACGAGGGGAAAT TAATAGGTTGTATTGATGTTGGACGAGTCGGAATCGCAGACCGATACCAGGATCTTGCCA TCCTATGGAACTGCCTCGGTGAGTTTTCTCCTTCATTACAGAAACGGCTTTTTCAAAAAT ATGGTATTGATAATCCTGATATGAATAAATTGCAGTTTCATTTGATGCTCGATGAGTTTT 
TCTAATCAGAATTGGTTAATTGGTTGTAACACTGGCAGAGCATTACGCTGACTTGACGGG ACGGCGCAAGCTCATGACCAAAATCCCTTAACGTGAGTTACGCGTCGTTCCACTGAGCGT CAGACCCCGTAGAAAAGATCAAAGGATCTTCTTGAGATCCTTTTTTTCTGCGCGTAATCT GCTGCTTGCAAACAAAAAAACCACCGCTACCAGCGGTGGTTTGTTTGCCGGATCAAGAGC TACCAACTCTTTTTCCGAAGGTAACTGGCTTCAGCAGAGCGCAGATACCAAATACTGTTC TTCTAGTGTAGCCGTAGTTAGGCCACCACTTCAAGAACTCTGTAGCACCGCCTACATACC TCGCTCTGCTAATCCTGTTACCAGTGGCTGCTGCCAGTGGCGATAAGTCGTGTCTTACCG GGTTGGACTCAAGACGATAGTTACCGGATAAGGCGCAGCGGTCGGGCTGAACGGGGGGTT CGTGCACACAGCCCAGCTTGGAGCGAACGACCTACACCGAACTGAGATACCTACAGCGTG AGCTATGAGAAAGCGCCACGCTTCCCGAAGGGAGAAAGGCGGACAGGTATCCGGTAAGCG GCAGGGTCGGAACAGGAGAGCGCACGAGGGAGCTTCCAGGGGGAAACGCCTGGTATCTTT ATAGTCCTGTCGGGTTTCGCCACCTCTGACTTGAGCGTCGATTTTTGTGATGCTCGTCAG GGGGGCGGAGCCTATGGAAAAACGCCAGCAACGCGGCCTTTTTACGGTTCCTGGCCTTTT GCTGGCCTTTTGCTCACATGTT 
Supplemental figure 2 
Name

$$
\text { pENT35H(24-3) }
$$

Type Entry Clone

Size $10481 \mathrm{bp}$

Origine BP cloning : pDONR35H x PCR (primers Cyp19a1a_B3 and Cyp19a1a_

\section{Selectable makers Kanamycin}

Bacterial strain DH5alpha

Comments

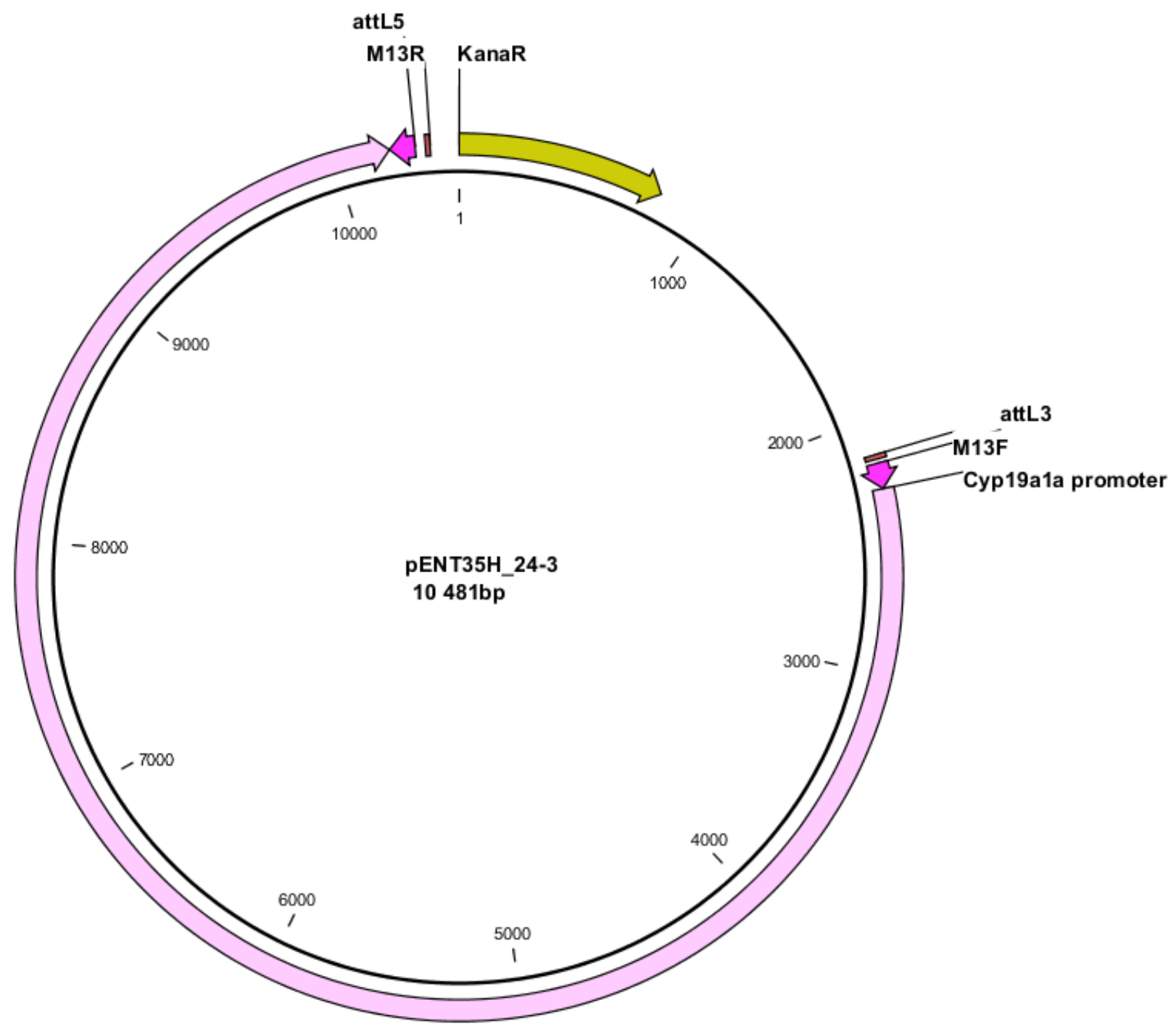




\section{Fasta sequence}

$>$ PENT35H_24-3

ATGAGCCATATTCAACGGGAAACGTCGAGGCCGCGATTAAATTCCAACATGGATGCTGATTTATATGGGTATA AATGGGCTCGCGATAATGTCGGGCAATCAGGTGCGACAATCTATCGCTTGTATGGGAAGCCCGATGCGCCAGA GTTGTTTCTGAAACATGGCAAAGGTAGCGTTGCCAATGATGTTACAGATGAGATGGTCAGACTAAACTGGCTG ACGGAATTTATGCCTCTTCCGACCATCAAGCATTTTATCCGTACTCCTGATGATGCATGGTTACTCACCACTG CGATCCCCGGAAAAACAGCATTCCAGGTATTAGAAGAATATCCTGATTCAGGTGAAAATATTGTTGATGCGCT GGCAGTGTTCCTGCGCCGGTTGCATTCGATTCCTGTTTGTAATTGTCCTTTTAACAGCGATCGCGTATTTCGT CTCGCTCAGGCGCAATCACGAATGAATAACGGTTTGGTTGATGCGAGTGATTTTGATGACGAGCGTAATGGCT GGCCTGTTGAACAAGTCTGGAAAGAAATGCATAAACTTTTGCCATTCTCACCGGATTCAGTCGTCACTCATGG TGATTTCTCACTTGATAACCTTATTTTTGACGAGGGGAAATTAATAGGTTGTATTGATGTTGGACGAGTCGGA ATCGCAGACCGATACCAGGATCTTGCCATCCTATGGAACTGCCTCGGTGAGTTTTCTCCTTCATTACAGAAAC GGCTTTTTCAAAAATATGGTATTGATAATCCTGATATGAATAAATTGCAGTTTCATTTGATGCTCGATGAGTT TTTCTAATCAGAATTGGTTAATTGGTTGTAACACTGGCAGAGCATTACGCTGACTTGACGGGACGGCGCAAGC TCATGACCAAAATCCCTTAACGTGAGTTACGCGTCGTTCCACTGAGCGTCAGACCCCGTAGAAAAGATCAAAG GATCTTCTTGAGATCCTTTTTTTCTGCGCGTAATCTGCTGCTTGCAAACAAAAAAACCACCGCTACCAGCGGT GGTTTGTTTGCCGGATCAAGAGCTACCAACTCTTTTTCCGAAGGTAACTGGCTTCAGCAGAGCGCAGATACCA AATACTGTTCTTCTAGTGTAGCCGTAGTTAGGCCACCACTTCAAGAACTCTGTAGCACCGCCTACATACCTCG CTCTGCTAATCCTGTTACCAGTGGCTGCTGCCAGTGGCGATAAGTCGTGTCTTACCGGGTTGGACTCAAGACG ATAGTTACCGGATAAGGCGCAGCGGTCGGGCTGAACGGGGGGTTCGTGCACACAGCCCAGCTTGGAGCGAACG ACCTACACCGAACTGAGATACCTACAGCGTGAGCTATGAGAAAGCGCCACGCTTCCCGAAGGGAGAAAGGCGG ACAGGTATCCGGTAAGCGGCAGGGTCGGAACAGGAGAGCGCACGAGGGAGCTTCCAGGGGGAAACGCCTGGTA TCTTTATAGTCCTGTCGGGTTTCGCCACCTCTGACTTGAGCGTCGATTTTTGTGATGCTCGTCAGGGGGGCGG AGCCTATGGAAAAACGCCAGCAACGCGGCCTTTTTACGGTTCCTGGCCTTTTGCTGGCCTTTTGCTCACATGT TCTTTCCTGCGTTATCCCCTGATTCTGTGGATAACCGTATTACCGCCTTTGAGTGAGCTGATACCGCTCGCCG CAGCCGAACGACCGAGCGCAGCGAGTCAGTGAGCGAGGAAGCGGAAGAGCGCCCAATACGCAAACCGCCTCTC CCCGCGCGTTGGCCGATTCATTAATGCAGCTGGCACGACAGGTTTCCCGACTGGAAAGCGGGCAGTGAGCGCA ACGCAATTAATACGCGTACCGCTAGCCAGGAAGAGTTTGTAGAAACGCAAAAAGGCCATCCGTCAGGATGGCC TTCTGCTTAGTTTGATGCCTGGCAGTTTATGGCGGGCGTCCTGCCCGCCACCCTCCGGGCCGTTGCTTCACAA CGTTCAAATCCGCTCCCGGCGGATTTGTCCTACTCAGGAGAGCGTTCACCGACAAACAACAGATAAAACGAAA GGCCCAGTCTTCCGACTGAGCCTTTCGTTTTATTTGATGCCTGGCAGTTCCCTACTCTCGCGTTAACGCTAGC ATGGATGTTTTCCCAGTCACGACGTTGTAAAACGACGGCCAGTCTTAAGCTCGGGCCCCCAAATAATGATTTT ATTTTGACTGATAGTGACCTGTTCGTTGCAACAAATTGATGAGCAATGCTTTTTTATAATGCCAACTTTGTAT AATAAAGTAGGCTCCTTTCTGATCAGGAGCAGATGTACTGATGAAATCACGTAAATTTGTACCAATTCGTATG AATTCGGTCATACAAAAATGTACAATTTTAAAAAGGAGGTGTGGTCCCCATCCCCGCCCCTAAACCCAAACAT CATTGGGGGGAAAAACAAATTGTGGAGGACAGAGGGCCTGAAACAGAGCTCTGAGGCACATCGCACTTGAACT TTTATAGAGACATTTACATTTACATTCAGTTATTTAGCAGACGCTTTTATCCAAAGCGACTTACAAATGAGGA CAATGAAAGCAATTAACACAACTATAAGAGCAACAATAAATAAGTGCTGTAGGCAAGTTTCAGGTGTGTAAAG AAAGTGTAAGAAGCAAAACATTAGTATTTTTTTATGTAGTTAGTGGAGGAGTCAGAGAGGGAATTGCATATTA GTAAGGAAAGTGGAAACTAAATAGTTGAGTTTTTTTGTTGTTTTTTGAGGACAGTGAGTGACTCTGCCGTTCT GATGCAGTTAGGGAGTTCATTCCACCAACTGGGCAGATTGAGCGCGAGAGTTCGGGAAAGTGATTTCTTCCCT CTTAGGGATGGAACCACGAGGCGACGTTCATTCGCAGAACTTAAGTTTCTGGAGGGCCTATAAATCTGCAGAA GTGAGAGCAGATAAGAAGCCAGAAATTGCTTTGTAAGCAAACATCAGAGCTTTAAATTTGAGCAACAACTGGC AGCCAGTGCAAACGGATAAGTAGCGGAGTGACATGTGCTCTTTTAGGTTCATTAAAGACCACTTGTGCTGCTG CGTTCTGAAGCATCTGAAGAGGTTTGATAGAGCTTGCTGGGAGCCCGGCTAGTAGAGAGTTGCAATAGTCCAG TCTGGAGAGAACAAGAGCTTGAACAAGGAGTTGAGCTGCATGTTCAGATAGGAAGGGTCGGATCTTTCTGATG TTGTAGAGTGCGAATCTGCATCGATCGAGCAGTTCTAGAGATGTGGTCAGAGAAGTTTAGCTGGTCATCAATC GTTACTACAAGGCTTTTTCCCATTTGTCCCATCCATCTGGATTGAAAAGTTATGATGTAGAGTCGGGTTGGCA GAAACAGGTCTCCATTTACACACAAAAAATAGGCAATAACCTATGAAAATTAAATAATAATAATAATAGAAAA CATAATGAAAATGTCTACGAGACAAATCCAGAAAAAGTAGAAATAATTATTTAACCATGTCAACTAAACAATA 
CTTGCACCATGTGTAAGACTTTTACATAACAAGACAAGACCACAGAGGGGTCAGTCGAGTGGACATTGGACTG TGCTCAGTGAAGGGAAGTGGTTTGGCTTCTGTCCCTGTGTGTTGTCTCAAACATTGCTGTTATTCTCTGTTAC TTGGCTCTCCCCATGAGAAAATGAACAAACCTCCACCCACAAGTCATACAGAATTAGACATAACCATCCCAGA GATCAGCACACATTGTGTGTGATGAGCTGCCATCAGTAATTAAGTTTAGGAACAATAAAAAAAAAATAAAAAA AAACATTATTGAAATGTTTGTCATAATTATGAAAAATTAAACAGAATATTTGTACTTCTTTTTTAACTATTAT TTTTCACAAAATGCATTTGCTGTGAACACAACGTAAACCTTTCTTACAAAGGAAGGACCCACCGGCAGCATAA TATACAGTAAGTGCGCTATAAATTTAATATGTCTGACAGTACCCACAATTCCCTTCCTTGTGGCTTTCTGCCC ACAGTTTGCTACAAGGGACAAACAACTTATTTGACGAATTATGAAACTCTAGGACCACAAAGGCCGTATTGAT GTGACGACATATTTAAGTGACGCAGTTTCCAGTGTGCAGAGGAACACTTTGTTCTGCGATGAATTAAAGATGC CTTGTTTTTTCTAAGAAAGACATTCCAGCAACAAATATGTCATGTGTGATGTTGAGAAGTTTATATCAGGAC CACCCGGAGATTCTGACATTCAAGGAAATTCCCTTTCCACCTCCAGTCTTTACTATAACTTATAGTACAAAAA AATTAAAATAATAACAATCTACTTTTAGTTGAAAAGAGACATGAACTGGCATTCAAATGTCACAGACACCTGA GGCATATTTGAAGGTCATCCAGTACCATAACAAAAAATAGTAACACATGTATTTGTAATGCAAAACATTTTGC CTTTTAAGGAGATATAATGCTGCGTATCATCTGTATAATCATAAAAATGAATTCCCTGCTTCCTTATGACATT CCAGAATTACAAAGCGCCTAAAACAGCGCCTTGTGGCACACCTTGATCACAATTTGCTCTTTACAAACAGACA AGTAGGCTTTTGGTAGCCCAATATTGTTTTTtTTAATACAAAATAAATATCTAGATCTAAATAAAACATCTAA CTAACTACCCAGAGGTTTAACCATAAAGTCACTATGAAATACTGACTTCTACAAATCTGCATTGTAATTCCTC CACATCATAATTATGAATTTAAATAAAAGGAAAATGAGGAAAGAAAACTCCTAAAGGGAACCTATTTTATGCA TTTTACAGTACGCAAGATAAGCCTTTGGTGTCTCTAGACTGTGTCTGTAAAGTTTCAGCTCTAAATACACATC ATCTTATCACAATGCTGGAGAAATTGTGAAGTAGGGGACTATGTTCACATATTTTAGGAATGTCCCAAAATAC AAGCGTATTGTTAAAAGGGAGATTAGTAAAGTATTAGGATACGACATATCAATGCACACAGTATTTTATTTTT ATtTTTTGCTTGATGGCTTCCCCTCTGAGCAGTTCAGCAAGTCTCACCTATTTGCACTATATGTATTATTAAT AACAGCTAGGAAGATAATTCCAGTGAAGTGGGTGAAGACCAGCTGACCTACTATGACAGAATGGACACAGACG CTGGAACAGATATACAGTTGTCAAAATTATTAGCCCCCCTGTTTATTTTTTCCCCTAATTTCTGTTTATCAGA GAGAAGATTTTTTTTAAAATACATTTCTAAACATAATAGTGTAATAACTCATTTCTAATAACTGATTTATTTT ATCTTTGCCGTGATGACAGTAAATTATATTTTACTAGATATTTTTCAAGACACTTTTATACAGTTTAAAgTAA CATTTAAAGGCTTAAGTCAGTTGATTAGGTTAACTAGGCGGGTTAGGGAAACTAGGCAAGTTATTGTACAACA ATGGTTTGTTCTGTAGACTATCAAACAAATATAGCTTAAAGGGGCTAATAATTCTGACCTTAAAATGGATTAA AAAAAAACTGCTTTTATTCTAGCCGAAATAAAACAAATAAGACTTTCTCCAGAAGAAAAGAAAATAATTAAAA AAGAAAAAAAAATCAAAGGGGGGCTATTCATTCTGACTTCAACTGTATATATTTAAAAACATGACTACAGATT TGCAGTCAAAATATTTTCCAACAGACCTGGGCATTAATCAAAACATACTGACTGGATCCTTGAAATGCGTTCT CAGGGGCTTTGTTGTAGTTATTGCCTTTATATTGAAGTTTATTTATCATTATTTTATATTATCAGTGATGTGT TCTGTATGAGATATCAAGGTTGTAAAATGAAGGTACCATACACACTCACCTCTTGGCGCACGTAATGCTGCAT TCATCTGCCCTGACTGTGCCCAAAGATGTCAGACTATTTCCATAAATTCTTTGGTGAAGAAAGTAGGCGTTTA GTGAATTCACAATGTCTCTGAATCTGATAGAATCACACAAAGAGGGCAAACTGAAGGCACAAATTAGCTTTTG TGCTCTCTGTAACAATTTGTTTTTGTTTTAAATTGAATGGTGAAAAACATTCAAATTCCTAAATAATCTTGAT GCTTTAAGACAGAATGCATAAAAAAAGAGGGGGGACAAATGATGACAAGATACATGTGGTACATCGGGTAATA TTTTACTTTGTGTCCACGATGAAGTATATAAATATGTATTTTTTTATTATAATGCATTATAGTGGTAAATGTG TCTGAACTGCCTATAATGTATTTATTTATTTATTTATTTATTTATTTTAAGAAATAAACGTAATAATGGATTT GTGGCATGTTTTTTTTTTTTTTTTTTTTTATTCCATGTCATGTTTTGCATTACCTAATGGTATAAGTCAGGT AAGTAAAATAAGTAGGCAAAATTTATTTGGTCACACTTTATTTTATTTTATTTTAATTCTCGAAATTTTCAAA GCATAAACCAAGACCTTTAGCTCAACAAAGTACTAATTTGCTACTGTTTGCTAATTTCTATCAAACTACTAAA CACTAAAAAAATGAATTTTGCTGCTTGTTCAAACTACCTATTTAAAATGGGTTAAAACAACACAATTACTTTG TGTTTTTTTATTTCATTTAAACTTAATGGTAATATGTTAAATCCACTTAAATATGTAAAAACGATTAAATTAA TCGATTTGTGTTGGGACAAAATAAAGTAATTGTGTGAAACGCGTTTTTTTTTTACTGTGTAGTTGTGTTAAGG TATTGGGTGTAGGATTAGGGATGTAGAATTAGATACTTTATAATTACTAATAAGTGGCCAATACCTTAATAAT AGGCAGTTAATAAGCCAGTAGGTAGCAGTGGAAATTGGTACATAAACCAAAGTGTTACCATTCGTTTATACTG TATAGTACATCTAACATTAAGTGATATACAATTTACACACTATTTCACATAAATATATCTGTGACATGGCATA ATGAGGGAGCTACTCAATGAACTGTTGAAAATATTGAAAATATTTAAATTCTGTTTCACAAAAAGTTACACAA AATATTAAATCACACACTTTACATCCATTTAAAATCACTTTTGTAAATGTATTTTAAAGCAGACTCCAAAATG GAGGCTTGACTTTGATCAATAAACATCCATTTTAGAAAAACAAAACTTAAAGATGCCTTTTACAGTACAGGAT TGTCAGTGACGAAGCAGATCCACGGTTGAAGGCAGAATAATTAGCCCTTGTTAATTATTATCCGCCCTGTGTA TTTCTGTTTAACGGAGAGAAGATTTTTTTCAACACATTTCTTAAGATAATAGTTTTAAGAACTAATTTCTAAT AACAGATTTTTCCTTATCTTTGCCATTATGACAGTACATAATATTTTAGATATTTTTCAAGATACTAGTATtT AGCTTAAAGTGTGATTTAAAGGCCTAACTAGTTAGGTTAACTTGGTGAGTTCGTTGTATAACAATGGTTTCAA 
ATtTtTTtTGCTTATATATAATGTAGGTCACACTTTACAATAAGGTTCATTAGTTAATGCTAAGTAATGCATT TACTAACATGAACAAACATTGAACAATACATTTACTACAGTATTTATTAATGTTAATAAACGTTAGTTAATGA AAATAGAATAATAGTGACCTTAAAATGGTTTAAAAACTAGCCAAAATAAAACAGATAAGACTTTCTTCAGAAG AAAAAAACATTACAGGAAATGTGAACATTTCAACAGAGGATTACTGTGAAAACATATAGCCGTATACATTTCT GAAGAGCATGAATTATGTAGCCAGAGCTACGTATGGCTGCATTTCCTCTTTAAAACGAACAATCCAGGGTGGT ATGATGCTGACGATAGATTTTGTTTTTTTTTACTCTTCCAGCTGACTGCTTACCTACGTGTGGGCGGCTTTTC TGCTGTTACCAGTTTGCCCAGTAGCTTGCCATGTCCATCGGTGGACTTTAGACAAAGAGAGGAGTTGACTGTG ACGACAGGGTTTGAGTCTGGTAAAGAATGGTTCCAGAAAGCAGCTAAAACCAAAACAAAAGGCAAAATTTATT TAGACAAGTACAGTTAAAACCGGAAGTTTACATACACTGCATAAAAAGGCACATAACCACTTAAAAAAAGTCA GATGTTAATGTAAACTTTTTCTCTTTTAGGTAAGTCAGGGTAATCAAATTTGTTTCTGTTATGCTTAATATCA GAATAATGAGGGAGATATTTTTGAGAAATTGTTATAACTTTTCTTGAAAGTCAAGTTTACATACAATAAGATT ATTATGCCTCTGAAAAAGCTCAGATGATGTTGTTACAACAGTAGAATAGTATTAAAGGAAAACCTCAACCACA CAGCTTCCTTGTGTGGCAACATGGGGAAATCTACAAGCCAGAATCAACAAAAAAGCCAGATTAAAATTAGCTA AATTGCACAGGGAAAAAGACACATTTTTGGAGACATGTCCTGTGGTCTGATGGAACTAAGATTGAACTGGTGG GCCATAATGATCTGTGTTACATTTGGAGGACAAAGGGGAAAGCTTACAAGCCTAGGAACACCATCCCAACTGT GAAGTATGAGGGCAGCAGCATCATCTTGTGAGGCTGTTTTACTGCGGGAGGGACTGGTCCACTTCACAGCATA GATGGCATCATGGACAAAGAACATTATGTTGAAATACTGAGTCAACATATCAAGACATCAGCCAGGAAATTAC AACTTGGCCACAAATGGGTCTTTCAAACAGACCATGACCCTAAGCATACTGCCAAATTAGTTCAAATATGCTT TAAGGACAACAGAGTGAATGTTTTAGAGTGGCCATCACAAAGCCCTGATCTCAATCCTATAGAAATTTGTAGG CAGCTTCTGTGAGCAAGACAGCCAACAAATCTGACTCAATTACACAGTTCTGTCAGGGGGAATGGGCCAAAAT TCCTTTTAGCTATTGTGAGAAGCTTGTGGAAGGACACCCAAAACATTTGACCAAAGTTAAACAGTTTAAAAGC AAAGCTAAAAAAATACATCTAACAAGGACATGTATGTAAACTTTTGACTGTCTAAAAAATTCTATCATTATTC TGGCATTTAGCAAATGTAATTAATTTAGGTAATCTTAACAGACCCAAAATAGTAAAGATTTAGTATGATTTAC CATCAGAAATTTCTTTAAAAAAAAGTTATGTTCCTTTTTTAAAGTGTATGAAAATTTGTGGATTCAACTGTAA ATAACAGGGTGAAAGTGTGGTAAAAACTAAAACTGTAGTAAAAATGAGGCAAGGCCTTTTTTGGATTGCTTTC GAAAACACTGTTGCTAAGGTTTAGGGAAGGGGTTAATCGGATCAATTGGTGCTTTTGAAAACACTATTGGTCG GGTTTAGGGTAAGGAATGGGGGGGGGGGTCAATTAGTCATTCAGTCAGTCAGTCGGTCAGTCGACAGCAGCCT CAGGTGGATTTATGCCAGAATAGTAGGTATGTATGTCACTCATTTGGGAGATCAGAAAAGGCACACACAGCAG TGGATTCTCGAAAACAAAAACTGCAAAAACTTGTTTCTCCTAGGATGTATTATGCAATATCCAGAAATGTATA TAAAGGGTACATATCAATAATGAGCCTGGATTGGAAAATAAATCCTTGCTCTGTTAAACATCATTTAAGAAAT ATTTGAAAAAGGGAAAAAAATTAAATAAAATCACAGGAGGGCTAATCATTTTGACTTCAACTGGATATAATGT GAGCAGCTTGTGAATGAATCTGCATTGCATTTACTGTAAAGCTTAACAGTCAAATGATAAATGAACATGATAA ATGAAGGTGCATCAAAATAAGGACACTTTTTGTTTGTTTGTAGGCCTGATGTTTTCTCATTTGACTGCTGTAG AATAACTCGAGGACGTGCCGCAGATGTCTAATATCCTTTCAAGTCTTTGAAGTCCTGATGAAAACCCAGAGAT GACTTGCACACAGCTGAGGGTTTGAGTGTCATGGTAAATGTTTTGGAAACTCGACGCTGAAGGACACAAAGGA GCACACAAGGTTACGCAGCGTTTTTATTTTGACTTTTGAATTATCCTGATTGAGTCCCATGCAGCTGCTGGGA TATAAAAGTGTACGTCTGCAGTTCAAAGGCTTTAGACTTGCTTCTTAATCCGTTCTTATGGCAGGACCCAACT TTTGTATACAAAGTTGGCATTATAAGAAAGCATTGCTTATCAATTTGTTGCAACGAACAGGTCACTATCAGTC AAAATAAAATCATTATTGGCCATCCAGCTCATATCCCCTATAGTGAGTCGTATTACATGGTCATAGCTGTTTC CTGGCAGCTCTGGCCCGTGTCTCAAAATCTCTGATGTTACATTGCACAAGATAAAATAATATCATCATGAACA ATAAAACTGTCTGCTTACATAAACAGTAATACAAGGGGTGTT 
Supplemental figure 3 
$\begin{array}{ll}\text { Name } & \text { pDESTGFP35H } \\ \text { Type } & \text { Destination Vector } \\ \text { Size } & 7895 \mathrm{bp} \\ \text { Origine } & \text { Genescript }+ \text { pDONR35H regeneration }\end{array}$

Selectable makers Ampicillin - chloramphenicol

Bacterial strain DB3.1(TM) or any [gyrA462]

Comments

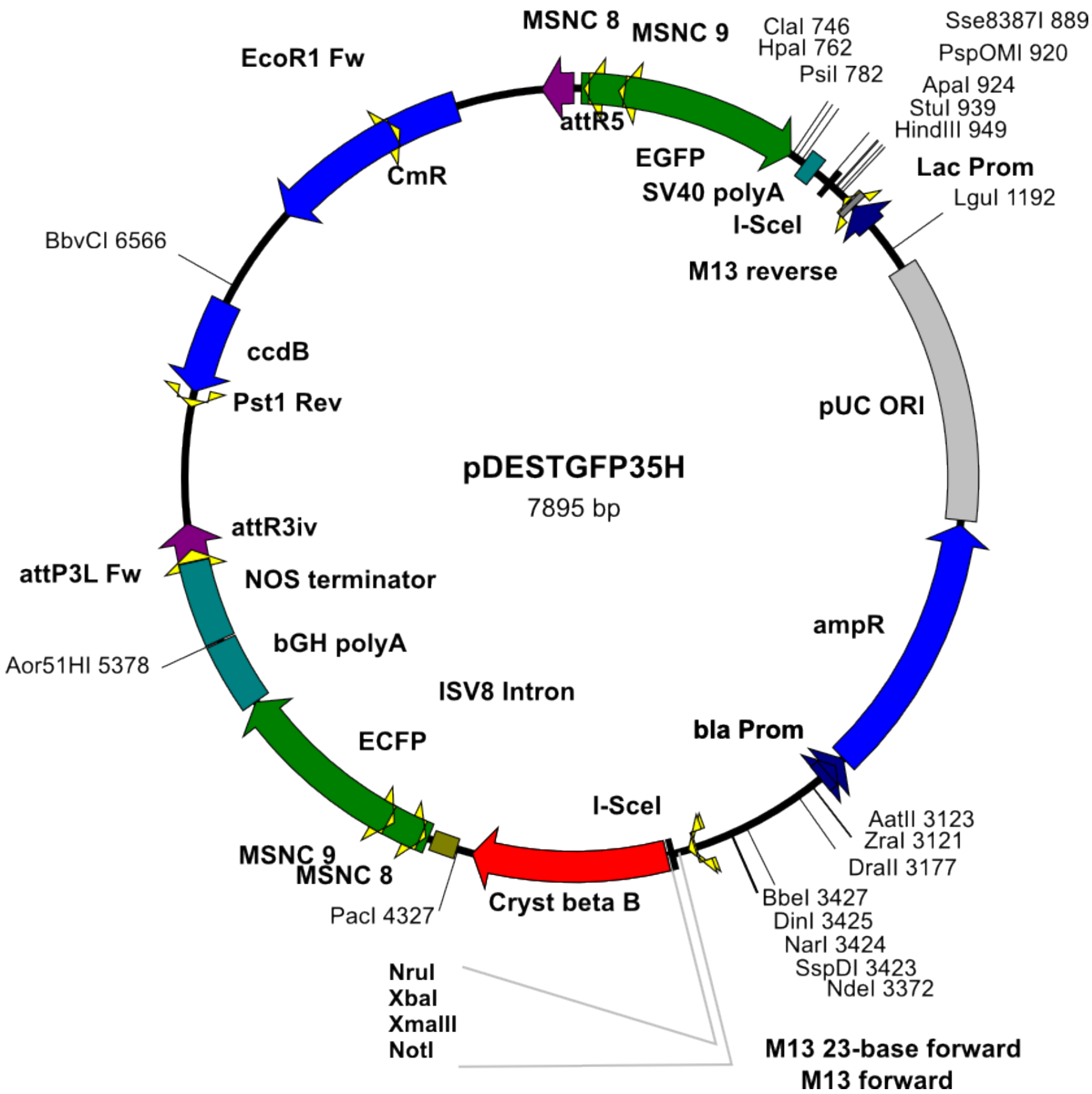




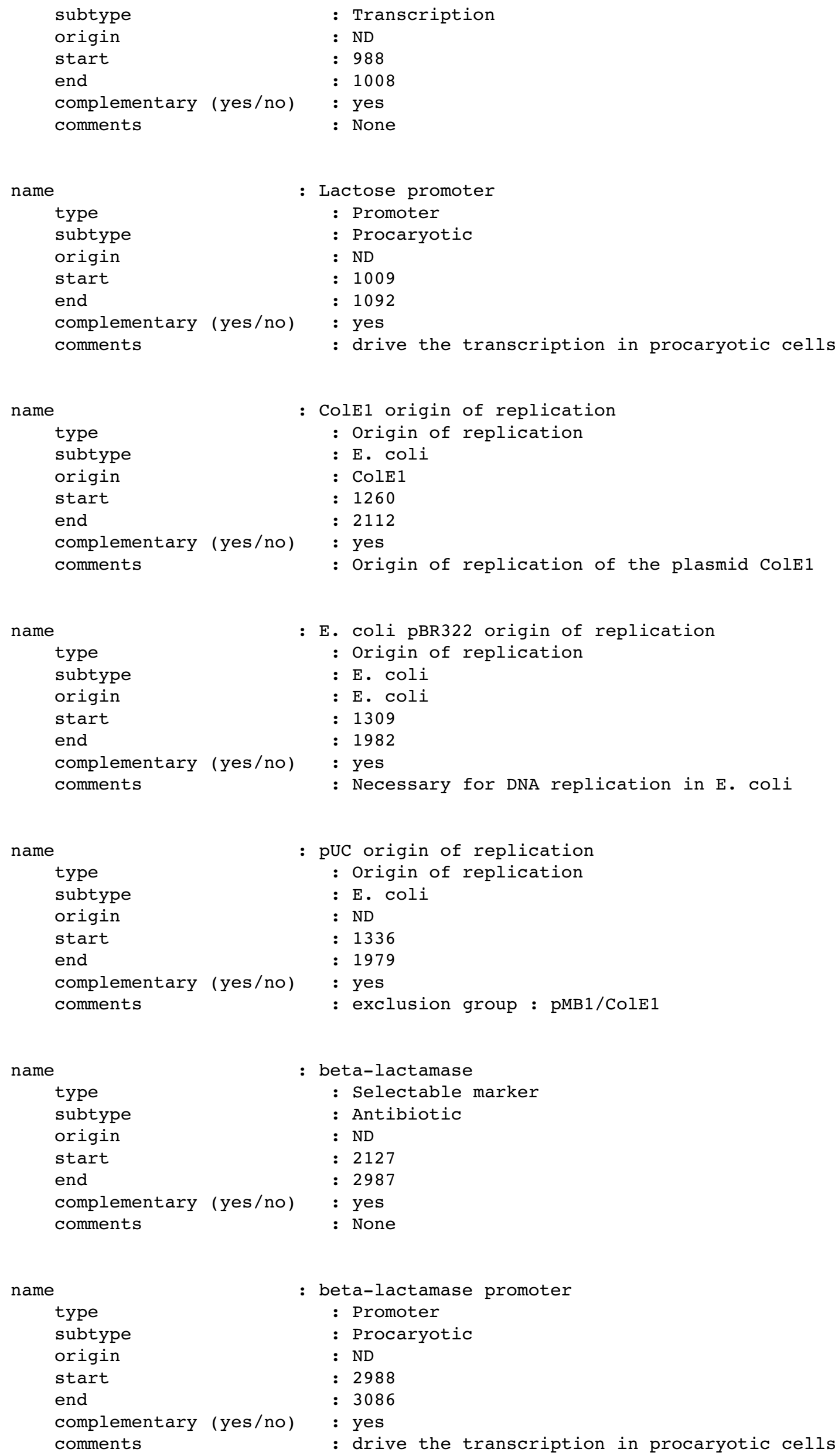




$\begin{array}{ll}\text { type } & : \text { Intron } \\ \text { subtype } & : \text { NA } \\ \text { origin } & : \text { synthetic } \\ \text { start } & : 4332 \\ \text { end } & : 4415 \\ \text { complementary (yes/no) } & : \text { no } \\ \text { comments } & : \text { Derived from pGene/V5-HisA(Invitrogen) }\end{array}$

name

type

subtype

origin

start

: Aequorea victoria Enhanced Cyan Fluorescent Protein

end

: Open Reading Frame

: NA

: Aequorea victoria

: 4430

: 5149

complementary (yes/no) : no

comments : Genetically engineered. Cyan derivative of the classical GFP. Humanized. excitation $\max =433 \mathrm{~nm}(+\operatorname{minor}$ peak $=453 \mathrm{~nm})$; emission $\max =475 \mathrm{~nm}$ $(+$ minor peak $=501 \mathrm{~nm})$. Sequence origin : Parinov \& Emylanov

name

type

subtype

origin

start

end

complementary (yes/no)

comments

: RP-GFP-1

: Binding site

: Primer

: ND

: 4438

: 4455

: yes

: $T m=65.5$. Orientation : Antisense. Target : CDNA of the EGFP.

name

type

subtype

origin

start

end

complementary (yes/no)

comments

: RP-GFP-2

: Binding site

: Primer

: ND

: 4551

: 4570

: yes

: $T m=63.9$. Orientation : Antisense. Target : CDNA of the EGFP.

name

type

subtype

origin

start

end

complementary (yes/no)

comments

: Bovine growth hormone polyadenylation signal

: Terminator of transcription

: polya

: Bovine

: 5150

: 5374

: no

: None

name

transcription

type

subtype

origin

start

end

Agrobacterium tumefaciens nopaline synthase terminator of

complementary (yes/no)

comments

: Terminator of transcription

: Bacterial

: Agrobacterium tumefaciens

: 5381

: 5647

: no

: None

name

type

subtype

: attR3iv

: Recombinaison binding site

: Gateway 
>pDESTGFP35H - 7895 bp - circular- Wed Jan 27 17:12:42 2010 ACTTTTGTATACAAAGTGGTACCATGGTGAGCAAGGGCGAGGAGCTGTTCACCGGGGTGG TGCCCATCCTGGTCGAGCTGGACGGCGACGTAAACGGCCACAAGTTCAGCGTGTCCGGCG AGGGCGAGGGCGATGCCACCTACGGCAAGCTGACCCTGAAGTTCATCTGCACCACCGGCA AGCTGCCCGTGCCCTGGCCCACCCTCGTGACCACCCTGACCTACGGCGTGCAGTGCTTCA GCCGCTACCCCGACCACATGAAGCAGCACGACTTCTTCAAGTCCGCCATGCCCGAAGGCT ACGTCCAGGAGCGCACCATCTTCTTCAAGGACGACGGCAACTACAAGACCCGCGCCGAGG TGAAGTTCGAGGGCGACACCCTGGTGAACCGCATCGAGCTGAAGGGCATCGACTTCAAGG AGGACGGCAACATCCTGGGGCACAAGCTGGAGTACAACTACAACAGCCACAACGTCTATA TCATGGCCGACAAGCAGAAGAACGGCATCAAGGTGAACTTCAAGATCCGCCACAACATCG AGGACGGCAGCGTGCAGCTCGCCGACCACTACCAGCAGAACACCCCCATCGGCGACGGCC CCGTGCTGCTGCCCGACAACCACTACCTGAGCACCCAGTCCGCCCTGAGCAAAGACCCCA ACGAGAAGCGCGATCACATGGTCCTGCTGGAGTTCGTGACCGCCGCCGGGATCACTCTCG GCATGGACGAGCTGTACAAGTAAATCGATATTGTTGTTGTTAACTTGTTTATTGCAGCTT ATAATGGTTACAAATAAAGCAATAGCATCACAAATTTCACAAATAAAGCATTTTTTTCAC TGCATTCTAGTTGTGGTTTGTCCAAACTCATCAATGTATCTTCCTGCAGGATTACCCTGT TATCCCTAATCGGATCCCGGGCCCGTCGACTGCAGAGGCCTGCATGCAAGCTTGGCGTAA TCATGGTCATAGCTGTTTCCTGTGTGAAATTGTTATCCGCTCACAATTCCACACAACATA CGAGCCGGAAGCATAAAGTGTAAAGCCTGGGGTGCCTAATGAGTGAGCTAACTCACATTA ATTGCGTTGCGCTCACTGCCCGCTTTCCAGTCGGGAAACCTGTCGTGCCAGCTGCATTAA TGAATCGGCCAACGCGCGGGGAGAGGCGGTTTGCGTATTGGGCGCTCTTCCGCTTCCTCG CTCACTGACTCGCTGCGCTCGGTCGTTCGGCTGCGGCGAGCGGTATCAGCTCACTCAAAG GCGGTAATACGGTTATCCACAGAATCAGGGGATAACGCAGGAAAGAACATGTGAGCAAAA GGCCAGCAAAAGGCCAGGAACCGTAAAAAGGCCGCGTTGCTGGCGTTTTTCCATAGGCTC CGCCCCCCTGACGAGCATCACAAAAATCGACGCTCAAGTCAGAGGTGGCGAAACCCGACA GGACTATAAAGATACCAGGCGTTTCCCCCTGGAAGCTCCCTCGTGCGCTCTCCTGTTCCG ACCCTGCCGCTTACCGGATACCTGTCCGCCTTTCTCCCTTCGGGAAGCGTGGCGCTTTCT CATAGCTCACGCTGTAGGTATCTCAGTTCGGTGTAGGTCGTTCGCTCCAAGCTGGGCTGT GTGCACGAACCCCCCGTTCAGCCCGACCGCTGCGCCTTATCCGGTAACTATCGTCTTGAG TCCAACCCGGTAAGACACGACTTATCGCCACTGGCAGCAGCCACTGGTAACAGGATTAGC AGAGCGAGGTATGTAGGCGGTGCTACAGAGTTCTTGAAGTGGTGGCCTAACTACGGCTAC ACTAGAAGAACAGTATTTGGTATCTGCGCTCTGCTGAAGCCAGTTACCTTCGGAAAAAGA GTTGGTAGCTCTTGATCCGGCAAACAAACCACCGCTGGTAGCGGTGGTTTTTTTGTTTGC AAGCAGCAGATTACGCGCAGAAAAAAAGGATCTCAAGAAGATCCTTTGATCTTTTCTACG GGGTCTGACGCTCAGTGGAACGAAAACTCACGTTAAGGGATTTTGGTCATGAGATTATCA AAAAGGATCTTCACCTAGATCCTTTTAAATTAAAAATGAAGTTTTAAATCAATCTAAAGT ATATATGAGTAAACTTGGTCTGACAGTTACCAATGCTTAATCAGTGAGGCACCTATCTCA GCGATCTGTCTATTTCGTTCATCCATAGTTGCCTGACTCCCCGTCGTGTAGATAACTACG ATACGGGAGGGCTTACCATCTGGCCCCAGTGCTGCAATGATACCGCGAGACCCACGCTCA CCGGCTCCAGATTTATCAGCAATAAACCAGCCAGCCGGAAGGGCCGAGCGCAGAAGTGGT CCTGCAACTTTATCCGCCTCCATCCAGTCTATTAATTGTTGCCGGGAAGCTAGAGTAAGT AGTTCGCCAGTTAATAGTTTGCGCAACGTTGTTGCCATTGCTACAGGCATCGTGGTGTCA CGCTCGTCGTTTGGTATGGCTTCATTCAGCTCCGGTTCCCAACGATCAAGGCGAGTTACA TGATCCCCCATGTTGTGCAAAAAAGCGGTTAGCTCCTTCGGTCCTCCGATCGTTGTCAGA AGTAAGTTGGCCGCAGTGTTATCACTCATGGTTATGGCAGCACTGCATAATTCTCTTACT GTCATGCCATCCGTAAGATGCTTTTCTGTGACTGGTGAGTACTCAACCAAGTCATTCTGA GAATAGTGTATGCGGCGACCGAGTTGCTCTTGCCCGGCGTCAATACGGGATAATACCGCG CCACATAGCAGAACTTTAAAAGTGCTCATCATTGGAAAACGTTCTTCGGGGCGAAAACTC TCAAGGATCTTACCGCTGTTGAGATCCAGTTCGATGTAACCCACTCGTGCACCCAACTGA TCTTCAGCATCTTTTACTTTCACCAGCGTTTCTGGGTGAGCAAAAACAGGAAGGCAAAAT GCCGCAAAAAAGGGAATAAGGGCGACACGGAAATGTTGAATACTCATACTCTTCCTTTTT CAATATTATTGAAGCATTTATCAGGGTTATTGTCTCATGAGCGGATACATATTTGAATGT ATTTAGAAAAATAAACAAATAGGGGTTCCGCGCACATTTCCCCGAAAAGTGCCACCTGAC GTCTAAGAAACCATTATTATCATGACATTAACCTATAAAAATAGGCGTATCACGAGGCCC TTTCGTCTCGCGCGTTTCGGTGATGACGGTGAAAACCTCTGACACATGCAGCTCCCGGAG ACGGTCACAGCTTGTCTGTAAGCGGATGCCGGGAGCAGACAAGCCCGTCAGGGCGCGTCA GCGGGTGTTGGCGGGTGTCGGGGCTGGCTTAACTATGCGGCATCAGAGCAGATTGTACTG AGAGTGCACCATATGCGGTGTGAAATACCGCACAGATGCGTAAGGAGAAAATACCGCATC AGGCGCCATTCGCCATTCAGGCTGCGCAACTGTTGGGAAGGGCGATCGGTGCGGGCCTCT TCGCTATTACGCCAGCTGGCGAAAGGGGGATGTGCTGCAAGGCGATTAAGTTGGGTAACG CCAGGGTTTTCCCAGTCACGACGTTGTAAAACGACGGCCAGTGAATTCGAGCTCGGTACC TCGCGAATGCATCTAGATTAGGGATAACAGGGTAATGCGGCCGCTCGAGCAATTGGTGCT ATCATCATGACAAAGAGAAAAGAAATCAGTTATTAGAAATGAGTTATTAAAACTATTATG ATTAGAAATATGTTTCTTTCTCTTAAACAGGATTTAAGGGGAAAATATACAGGGAGCGAA AAATTTTGACTTCATCTGTATATACATATAATAAATAATACATCAATAAATTCCATCTAA ATACGCTACCATATATATATATATATGTATATACACACACACACACACACCATCTAATAT 
ATGTCATGCTACATTATTAACTTCAGTATGAAATCTACTCTGCTCTGGAGTGTATCAGCG AGCGACTCCTCTGCTCGGATTATCATCACGTGACCTCTGACCTGAACACAGACCTCTCTT AGCCATAGGTATACAGCCTATAGCATAGAGCTCGGGCTGAGAGGGAAAGTGCCGCCGTCA GGGCTTTCCATTGACAGTAAAATGCTTACGGCCTTCTGGGCGATGCGATGACCCTGCGCT TCGGCACGGCTGAACTCTGATGACGGCGTGTTTCTATTGTCCGCCGCGCGCCGCTGTGCT GCCCTTGCCAGCGTATAAAAGCTGCGGCAGTGGTGAGGAAGGCCAGAGCGAGCCAGCCTT CCCAGCACAGCTGTAGTTTTAGGTTGCCGGGAACGGTGCATTGGAACGCGCATTCCCCGT GTTAATTAACAGGTAAGTGTCTTCCTCCTGTTTCCTTCCCCTGCTATTCTGCTCAACCTT CCTATCAGAAACTGCAGTATCTGTATTTTTGCTAGGGGATCCCGCCACCATGGTGAGCAA GGGCGAGGAGCTGTTCACCGGGGTGGTGCCCATCCTGGTCGAGCTGGACGGCGACGTAAA CGGCCACAAGTTCAGCGTGTCCGGCGAGGGCGAGGGCGATGCCACCTACGGCAAGCTGAC CCTGAAGTTCATCTGCACCACCGGCAAGCTGCCCGTGCCCTGGCCCACCCTCGTGACCAC CCTGACCTGGGGCGTGCAGTGCTTCAGCCGCTACCCCGACCACATGAAGCAGCACGACTT СTTCAAGTCCGCCATGCCCGAAGGCTACGTCCAGGAGCGCACCATCTTCTTCAAGGACGA CGGCAACTACAAGACCCGCGCCGAGGTGAAGTTCGAGGGCGACACCCTGGTGAACCGCAT CGAGCTGAAGGGCATCGACTTCAAGGAGGACGGCAACATCCTGGGGCACAAGCTGGAGTA CAACTACATCAGCCACAACGTCTATATCACCGCCGACAAGCAGAAGAACGGCATCAAGGC CAACTTCAAGATCCGCCACAACATCGAGGACGGCAGCGTGCAGCTCGCCGACCACTACCA GCAGAACACCCCCATCGGCGACGGCCCCGTGCTGCTGCCCGACAACCACTACCTGAGCAC CCAGTCCGCCCTGAGCAAAGACCCCAACGAGAAGCGCGATCACATGGTCCTGCTGGAGTT CGTGACCGCCGCCGGGATCACTCTCGGCATGGACGAGCTGTACAAGTAACTGTGCCTTCT AGTTGCCAGCCATCTGTTGTTTGCCCCTCCCCCGTGCCTTCCTTGACCCTGGAAGGTGCC ACTCCCACTGTCCTTTCCTAATAAAATGAGGAAATTGCATCGCATTGTCTGAGTAGGTGT САTTCTATTCTGGGGGGTGGGGTGGGGCAGGACAGCAAGGGGGAGGATTGGGAAGACAAT AGCAGGCATGCTGGGGATGCGGTGGGCTCTATGGAGCGCTTTAAGATTGAATCCTGTTGC CGGTCTTGCGATGATTATCATATAATTTCTGTTGAATTACGTTAAGCATGTAATAATTAA CATGTAATGCATGACGTTATTTATGAGATGGGTTTTTATGATTAGAGTCCCGCAATTATA CATTTAATACGCGATAGAAAACAAAATATAGCGCGCAAACTAGGATAAATTATCGCGCGC GGTGTCATCTATGTTACTAGATCGGGAATTGATCCCCCCTCGACAGCTTGCATGCCGCTT GGGCTGCACAACTTTGTATAATAAAGTTGAACGAGAAACGTAAAATGATATAAATATCAA TATATTAAATTAGATTTTGCATAAAAAACAGACTACATAATACTGTAAAACACAACATAT CCAGTCACTATGAATCAACTACTTAGATGGTATTAGTGACCTGTAGTCGACCGACAGCCT TCCAAATGTTCTTCGGGTGATGCTGCCAACTTAGTCGACCGACAGCCTTCCAAATGTTCT TCTCAAACGGAATCGTCGTATCCAGCCTACTCGCTATTGTCCTCAATGCCGTATTAAATC ATAAAAAGAAATAAGAAAAAGAGGTGCGAGCCTCTTTTTTGTGTGACAAAATAAAAACAT CTACCTATTCATATACGCTAGTGTCATAGTCCTGAAAATCATCTGCATCAAGAACAATTT CACAACTCTTATACTTTTCTCTTACAAGTCGTTCGGCTTCATCTGGATTTTCAGCCTCTA TACTTACTAAACGTGATAAAGTTTCTGTAATTTCTACTGTATCGACCTGCAGACTGGCTG TGTATAAGGGAGCCTGACATTTATATTCCCCAGAACATCAGGTTAATGGCGTTTTTGATG TCATTTTCGCGGTGGCTGAGATCAGCCACTTCTTCCCCGATAACGGAGACCGGCACACTG GCCATATCGGTGGTCATCATGCGCCAGCTTTCATCCCCGATATGCACCACCGGGTAAAGT TCACGGGAGACTTTATCTGACAGCAGACGTGCACTGGCCAGGGGGATCACCATCCGTCGC CCGGGCGTGTCAATAATATCACTCTGTACATCCACAAACAGACGATAACGGCTCTCTCTT TTATAGGTGTAAACCTTAAACTGCATTTCACCAGCCCCTGTTCTCGTCAGCAAAAGAGCC GTTCATTTCAATAAACCGGGCGACCTCAGCCATCCCTTCCTGATTTTCCGCTTTCCAGCG TTCGGCACGCAGACGACGGGCTTCATTCTGCATGGTTGTGCTTACCAGACCGGAGATATT GACATCATATATGCCTTGAGCAACTGATAGCTGTCGCTGTCAACTGTCACTGTAATACGC TGCTTCATAGCATACCTCTTTTTGACATACTTCGGGTATACATATCAGTATATATTCTTA TACCGCAAAAATCAGCGCGCAAATACGCATACTGTTATCTGGCTTTTAGTAAGCCGGATC CACGCGGCGTTTACGCCCCGCCCTGCCACTCATCGCAGTACTGTTGTAATTCATTAAGCA TTCTGCCGACATGGAAGCCATCACAGACGGCATGATGAACCTGAATCGCCAGCGGCATCA GCACCTTGTCGCCTTGCGTATAATATTTGCCCATGGTGAAAACGGGGGCGAAGAAGTTGT CCATATTGGCCACGTTTAAATCAAAACTGGTGAAACTCACCCAGGGATTGGCTGAGACGA AAAACATATTCTCAATAAACCCTTTAGGGAAATAGGCCAGGTTTTCACCGTAACACGCCA CATCTTGCGAATATATGTGTAGAAACTGCCGGAAATCGTCGTGGTATTCACTCCAGAGCG ATGAAAACGTTTCAGTTTGCTCATGGAAAACGGTGTAACAAGGGTGAACACTATCCCATA TCACCAGCTCACCGTCTTTCATTGCCATACGGAATTCCGGATGAGCATTCATCAGGCGGG CAAGAATGTGAATAAAGGCCGGATAAAACTTGTGCTTATTTTTCTTTACGGTCTTTAAAA AGGCCGTAATATCCAGCTGAACGGTCTGGTTATAGGTACATTGAGCAACTGACTGAAATG CCTCAAAATGTTCTTTACGATGCCATTGGGATATATCAACGGTGGTATATCCAGTGATTT TTTTCTCCATTTTAGCTTCCTTAGCTCCTGAAAATCTCGATAACTCAAAAAATACGCCCG GTAGTGATCTTATTTCATTATGGTGAAAGTTGGAACCTCTTACGTGCCGATCAACGTCTC ATTTTCGCCAAAAGTTGGCCCAGGGCTTCCCGGTATCAACAGGGACACCAGGATTTATTT ATTCTGCGAAGTGATCTTCCGTCACAGGTATTTATTCGGCGCAAAGTGCGTCGGGTGATG CTGCCAACTTAGTCGACTACAGGTCACTAATACCATCTAAGTAGTTGATTCATAGTGACT GGATATGTTGTGTTTTACAGTATTATGTAGTCTGTTTTTTATGCAAAATCTAATTTAATA TATTGATATTTATATCATTTTACGTTTCTCGTTCA 
Supplemental figure 4 
Name

pEXP35H(24-3)

Type

Expression Vector

Size

$13598 \mathrm{bp}$

Origine

LR cloning : pENTR35H (24-3) x pDESTGFP35H

Selectable makers Ampicillin

Bacterial strain Top10

Comments Zebrafish selectable marker : expression of CFP in the lens from 48 hours onward

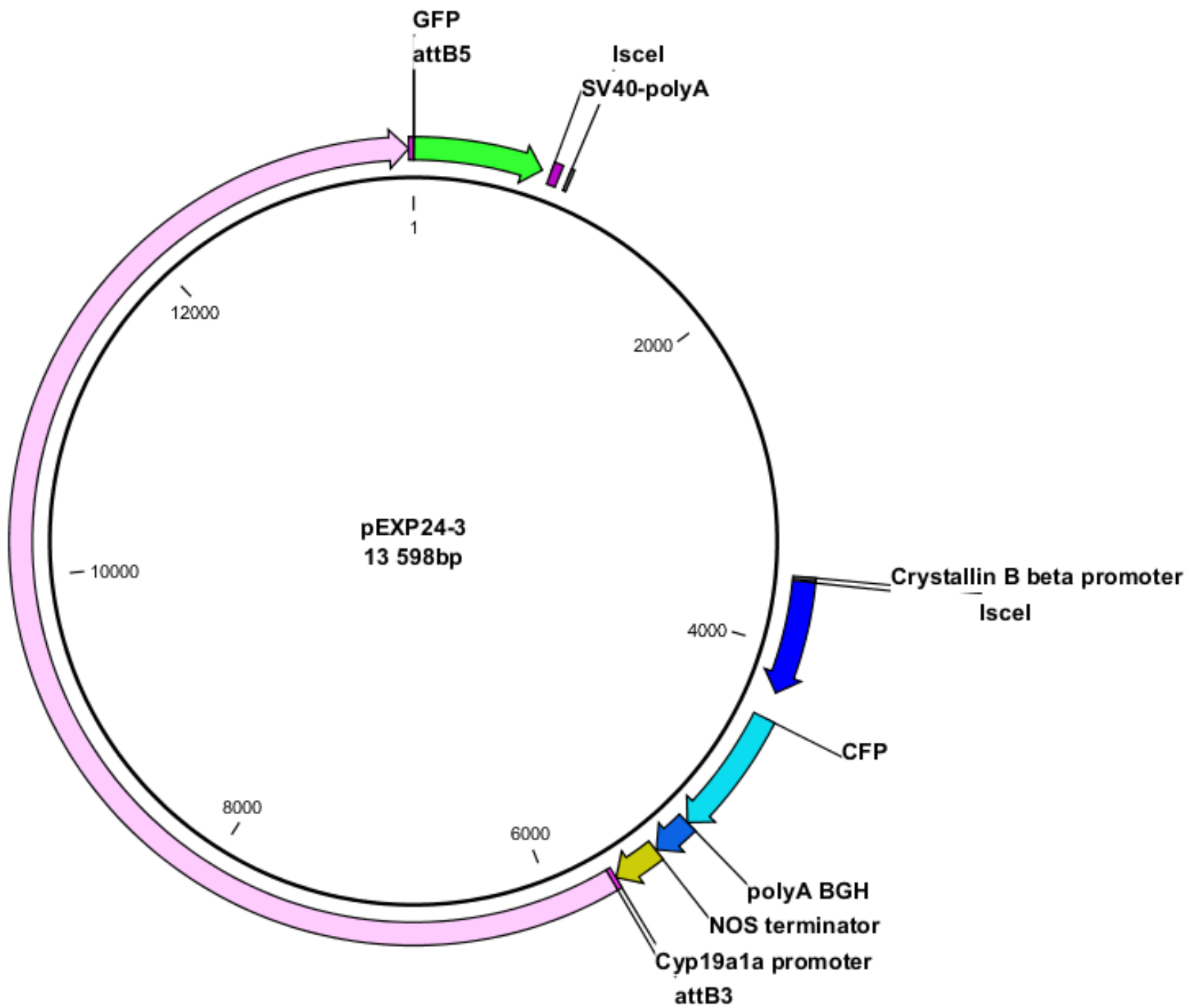

$\underline{\operatorname{pExP} 35 \mathrm{H} \quad 24-3 \operatorname{map}}$ 


\section{Features}

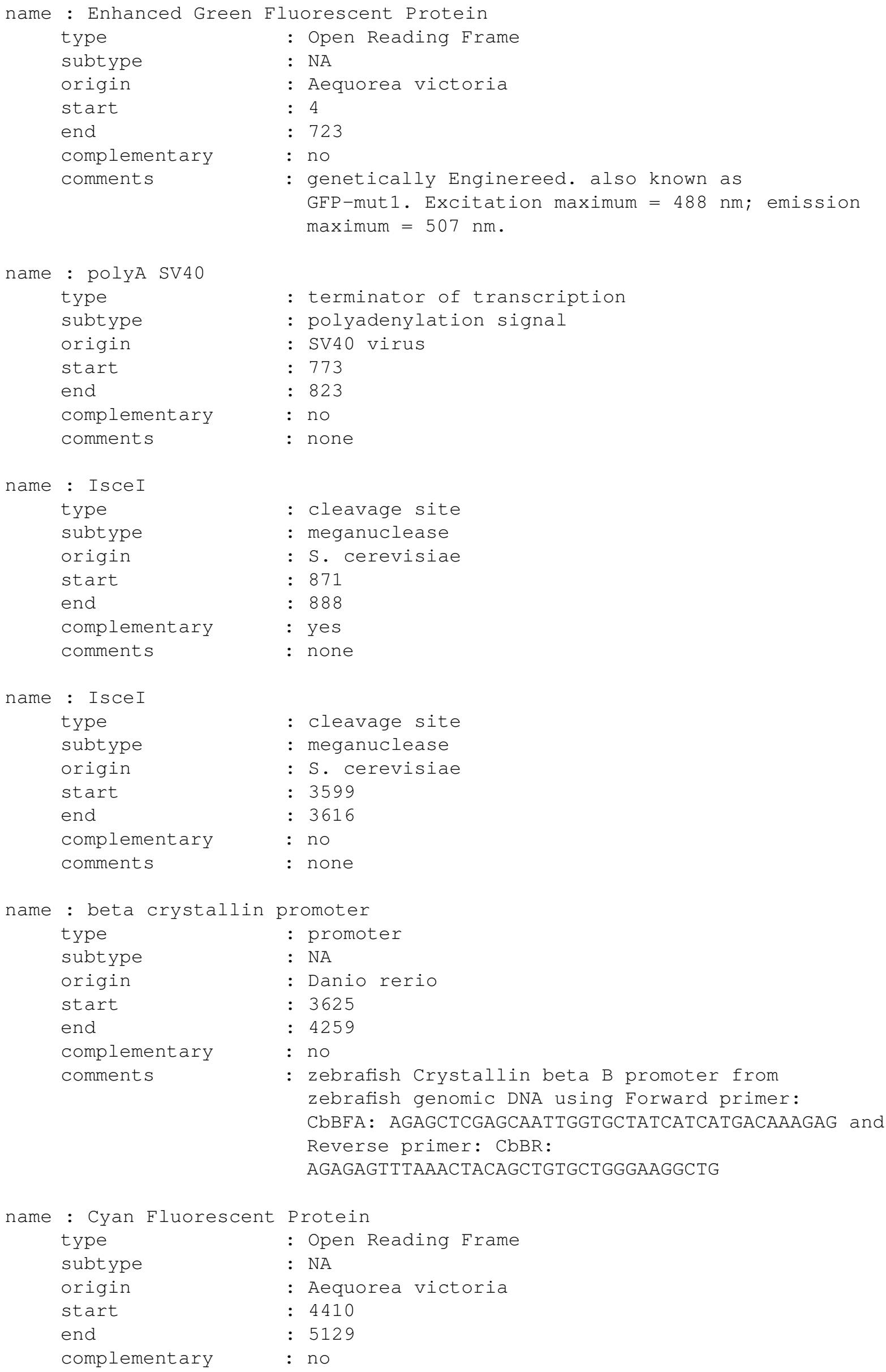


comments

the

: genetically engineered. Cyan derivative of

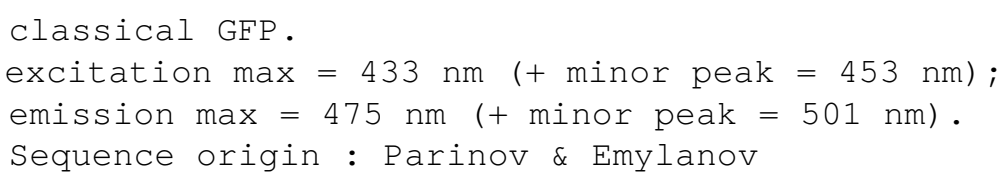

Fasta sequence

$>$ PEXP35H_24-3

ACCATGGTGAGCAAGGGCGAGGAGCTGTTCACCGGGGTGGTGCCCATCCTGGTCGAGCTGGACGGCGACGTAA ACGGCCACAAGTTCAGCGTGTCCGGCGAGGGCGAGGGCGATGCCACCTACGGCAAGCTGACCCTGAAGTTCAT CTGCACCACCGGCAAGCTGCCCGTGCCCTGGCCCACCCTCGTGACCACCCTGACCTACGGCGTGCAGTGCTTC AGCCGCTACCCCGACCACATGAAGCAGCACGACTTCTTCAAGTCCGCCATGCCCGAAGGCTACGTCCAGGAGC GCACCATCTTCTTCAAGGACGACGGCAACTACAAGACCCGCGCCGAGGTGAAGTTCGAGGGCGACACCCTGGT GAACCGCATCGAGCTGAAGGGCATCGACTTCAAGGAGGACGGCAACATCCTGGGGCACAAGCTGGAGTACAAC TACAACAGCCACAACGTCTATATCATGGCCGACAAGCAGAAGAACGGCATCAAGGTGAACTTCAAGATCCGCC ACAACATCGAGGACGGCAGCGTGCAGCTCGCCGACCACTACCAGCAGAACACCCCCATCGGCGACGGCCCCGT GCTGCTGCCCGACAACCACTACCTGAGCACCCAGTCCGCCCTGAGCAAAGACCCCAACGAGAAGCGCGATCAC 
ATGGTCCTGCTGGAGTTCGTGACCGCCGCCGGGATCACTCTCGGCATGGACGAGCTGTACAAGTAAATCGATA TTGTTGTTGTTAACTTGTTTATTGCAGCTTATAATGGTTACAAATAAAGCAATAGCATCACAAATTTCACAAA TAAAGCATTTTTTTCACTGCATTCTAGTTGTGGTTTGTCCAAACTCATCAATGTATCTTCCTGCAGGATTACC CTGTTATCCCTAATCGGATCCCGGGCCCGTCGACTGCAGAGGCCTGCATGCAAGCTTGGCGTAATCATGGTCA TAGCTGTTTCCTGTGTGAAATTGTTATCCGCTCACAATTCCACACAACATACGAGCCGGAAGCATAAAGTGTA AAGCCTGGGGTGCCTAATGAGTGAGCTAACTCACATTAATTGCGTTGCGCTCACTGCCCGCTTTCCAGTCGGG AAACCTGTCGTGCCAGCTGCATTAATGAATCGGCCAACGCGCGGGGAGAGGCGGTTTGCGTATTGGGCGCTCT TCCGCTTCCTCGCTCACTGACTCGCTGCGCTCGGTCGTTCGGCTGCGGCGAGCGGTATCAGCTCACTCAAAGG CGGTAATACGGTTATCCACAGAATCAGGGGATAACGCAGGAAAGAACATGTGAGCAAAAGGCCAGCAAAAGGC CAGGAACCGTAAAAAGGCCGCGTTGCTGGCGTTTTTCCATAGGCTCCGCCCCCCTGACGAGCATCACAAAAAT CGACGCTCAAGTCAGAGGTGGCGAAACCCGACAGGACTATAAAGATACCAGGCGTTTCCCCCTGGAAGCTCCC TCGTGCGCTCTCCTGTTCCGACCCTGCCGCTTACCGGATACCTGTCCGCCTTTCTCCCTTCGGGAAGCGTGGC GCTTTCTCATAGCTCACGCTGTAGGTATCTCAGTTCGGTGTAGGTCGTTCGCTCCAAGCTGGGCTGTGTGCAC GAACCCCCCGTTCAGCCCGACCGCTGCGCCTTATCCGGTAACTATCGTCTTGAGTCCAACCCGGTAAGACACG ACTTATCGCCACTGGCAGCAGCCACTGGTAACAGGATTAGCAGAGCGAGGTATGTAGGCGGTGCTACAGAGTT CTTGAAGTGGTGGCCTAACTACGGCTACACTAGAAGAACAGTATTTGGTATCTGCGCTCTGCTGAAGCCAGTT ACCTTCGGAAAAAGAGTTGGTAGCTCTTGATCCGGCAAACAAACCACCGCTGGTAGCGGTGGTTTTTTTGTTT GCAAGCAGCAGATTACGCGCAGAAAAAAAGGATCTCAAGAAGATCCTTTGATCTTTTCTACGGGGTCTGACGC TCAGTGGAACGAAAACTCACGTTAAGGGATTTTGGTCATGAGATTATCAAAAAGGATCTTCACCTAGATCCTT TTAAATTAAAAATGAAGTTTTAAATCAATCTAAAGTATATATGAGTAAACTTGGTCTGACAGTTACCAATGCT TAATCAGTGAGGCACCTATCTCAGCGATCTGTCTATTTCGTTCATCCATAGTTGCCTGACTCCCCGTCGTGTA GATAACTACGATACGGGAGGGCTTACCATCTGGCCCCAGTGCTGCAATGATACCGCGAGACCCACGCTCACCG GCTCCAGATTTATCAGCAATAAACCAGCCAGCCGGAAGGGCCGAGCGCAGAAGTGGTCCTGCAACTTTATCCG CCTCCATCCAGTCTATTAATTGTTGCCGGGAAGCTAGAGTAAGTAGTTCGCCAGTTAATAGTTTGCGCAACGT TGTTGCCATTGCTACAGGCATCGTGGTGTCACGCTCGTCGTTTGGTATGGCTTCATTCAGCTCCGGTTCCCAA CGATCAAGGCGAGTTACATGATCCCCCATGTTGTGCAAAAAAGCGGTTAGCTCCTTCGGTCCTCCGATCGTTG TCAGAAGTAAGTTGGCCGCAGTGTTATCACTCATGGTTATGGCAGCACTGCATAATTCTCTTACTGTCATGCC ATCCGTAAGATGCTTTTCTGTGACTGGTGAGTACTCAACCAAGTCATTCTGAGAATAGTGTATGCGGCGACCG AGTTGCTCTTGCCCGGCGTCAATACGGGATAATACCGCGCCACATAGCAGAACTTTAAAAGTGCTCATCATTG GAAAACGTTCTTCGGGGCGAAAACTCTCAAGGATCTTACCGCTGTTGAGATCCAGTTCGATGTAACCCACTCG TGCACCCAACTGATCTTCAGCATCTTTTACTTTCACCAGCGTTTCTGGGTGAGCAAAAACAGGAAGGCAAAAT GCCGCAAAAAAGGGAATAAGGGCGACACGGAAATGTTGAATACTCATACTCTTCCTTTTTCAATATTATTGAA GCATTTATCAGGGTTATTGTCTCATGAGCGGATACATATTTGAATGTATTTAGAAAAATAAACAAATAGGGGT TCCGCGCACATTTCCCCGAAAAGTGCCACCTGACGTCTAAGAAACCATTATTATCATGACATTAACCTATAAA AATAGGCGTATCACGAGGCCCTTTCGTCTCGCGCGTTTCGGTGATGACGGTGAAAACCTCTGACACATGCAGC TCCCGGAGACGGTCACAGCTTGTCTGTAAGCGGATGCCGGGAGCAGACAAGCCCGTCAGGGCGCGTCAGCGGG TGTTGGCGGGTGTCGGGGCTGGCTTAACTATGCGGCATCAGAGCAGATTGTACTGAGAGTGCACCATATGCGG TGTGAAATACCGCACAGATGCGTAAGGAGAAAATACCGCATCAGGCGCCATTCGCCATTCAGGCTGCGCAACT GTTGGGAAGGGCGATCGGTGCGGGCCTCTTCGCTATTACGCCAGCTGGCGAAAGGGGGATGTGCTGCAAGGCG ATTAAGTTGGGTAACGCCAGGGTTTTCCCAGTCACGACGTTGTAAAACGACGGCCAGTGAATTCGAGCTCGGT ACCTCGCGAATGCATCTAGATTAGGGATAACAGGGTAATGCGGCCGCTCGAGCAATTGGTGCTATCATCATGA CAAAGAGAAAAGAAATCAGTTATTAGAAATGAGTTATTAAAACTATTATGATTAGAAATATGTTTCTTTCTCT TAAACAGGATTTAAGGGGAAAATATACAGGGAGCGAAAAATTTTGACTTCATCTGTATATACATATAATAAAT AATACATCAATAAATTCCATCTAAATACGCTACCATATATATATATATATGTATATACACACACACACACACA CCATCTAATATATGTCATGCTACATTATTAACTTCAGTATGAAATCTACTCTGCTCTGGAGTGTATCAGCGAG CGACTCCTCTGCTCGGATTATCATCACGTGACCTCTGACCTGAACACAGACCTCTCTTAGCCATAGGTATACA GCCTATAGCATAGAGCTCGGGCTGAGAGGGAAAGTGCCGCCGTCAGGGCTTTCCATTGACAGTAAAATGCTTA CGGCCTTCTGGGCGATGCGATGACCCTGCGCTTCGGCACGGCTGAACTCTGATGACGGCGTGTTTCTATTGTC CGCCGCGCGCCGCTGTGCTGCCCTTGCCAGCGTATAAAAGCTGCGGCAGTGGTGAGGAAGGCCAGAGCGAGCC AGCCTTCCCAGCACAGCTGTAGTTTTAGGTTGCCGGGAACGGTGCATTGGAACGCGCATTCCCCGTGTTAATT AACAGGTAAGTGTCTTCCTCCTGTTTCCTTCCCCTGCTATTCTGCTCAACCTTCCTATCAGAAACTGCAGTAT CTGTATTTTTGCTAGGGGATCCCGCCACCATGGTGAGCAAGGGCGAGGAGCTGTTCACCGGGGTGGTGCCCAT CCTGGTCGAGCTGGACGGCGACGTAAACGGCCACAAGTTCAGCGTGTCCGGCGAGGGCGAGGGCGATGCCACC TACGGCAAGCTGACCCTGAAGTTCATCTGCACCACCGGCAAGCTGCCCGTGCCCTGGCCCACCCTCGTGACCA CCCTGACCTGGGGCGTGCAGTGCTTCAGCCGCTACCCCGACCACATGAAGCAGCACGACTTCTTCAAGTCCGC 
CATGCCCGAAGGCTACGTCCAGGAGCGCACCATCTTCTTCAAGGACGACGGCAACTACAAGACCCGCGCCGAG GTGAAGTTCGAGGGCGACACCCTGGTGAACCGCATCGAGCTGAAGGGCATCGACTTCAAGGAGGACGGCAACA TCCTGGGGCACAAGCTGGAGTACAACTACATCAGCCACAACGTCTATATCACCGCCGACAAGCAGAAGAACGG CATCAAGGCCAACTTCAAGATCCGCCACAACATCGAGGACGGCAGCGTGCAGCTCGCCGACCACTACCAGCAG AACACCCCCATCGGCGACGGCCCCGTGCTGCTGCCCGACAACCACTACCTGAGCACCCAGTCCGCCCTGAGCA AAGACCCCAACGAGAAGCGCGATCACATGGTCCTGCTGGAGTTCGTGACCGCCGCCGGGATCACTCTCGGCAT GGACGAGCTGTACAAGTAACTGTGCCTTCTAGTTGCCAGCCATCTGTTGTTTGCCCCTCCCCCGTGCCTTCCT TGACCCTGGAAGGTGCCACTCCCACTGTCCTTTCCTAATAAAATGAGGAAATTGCATCGCATTGTCTGAGTAG GTGTCATTCTATTCTGGGGGGTGGGGTGGGGCAGGACAGCAAGGGGGAGGATTGGGAAGACAATAGCAGGCAT GCTGGGGATGCGGTGGGCTCTATGGAGCGCTTTAAGATTGAATCCTGTTGCCGGTCTTGCGATGATTATCATA TAATTTCTGTTGAATTACGTTAAGCATGTAATAATTAACATGTAATGCATGACGTTATTTATGAGATGGGTTT TTATGATTAGAGTCCCGCAATTATACATTTAATACGCGATAGAAAACAAAATATAGCGCGCAAACTAGGATAA ATTATCGCGCGCGGTGTCATCTATGTTACTAGATCGGGAATTGATCCCCCCTCGACAGCTTGCATGCCGCTTG GGCTGCACAAGTTTGTATAATAAAGTAGGCTCCTTTCTGATCAGGAGCAGATGTACTGATGAAATCACGTAAA TTTGTACCAATTCGTATGAATTCGGTCATACAAAAATGTACAATTTTAAAAAGGAGGTGTGGTCCCCATCCCC GCCCCTAAACCCAAACATCATTGGGGGGAAAAACAAATTGTGGAGGACAGAGGGCCTGAAACAGAGCTCTGAG GCACATCGCACTTGAACTTTTATAGAGACATTTACATTTACATTCAGTTATTTAGCAGACGCTTTTATCCAAA GCGACTTACAAATGAGGACAATGAAAGCAATTAACACAACTATAAGAGCAACAATAAATAAGTGCTGTAGGCA AGTTTCAGGTGTGTAAAGAAAGTGTAAGAAGCAAAACATTAGTATTTTTTTATGTAGTTAGTGGAGGAGTCAG AGAGGGAATTGCATATTAGTAAGGAAAGTGGAAACTAAATAGTTGAGTTTTTTTGTTGTTTTTTGAGGACAGT GAGTGACTCTGCCGTTCTGATGCAGTTAGGGAGTTCATTCCACCAACTGGGCAGATTGAGCGCGAGAGTTCGG GAAAGTGATTTCTTCCCTCTTAGGGATGGAACCACGAGGCGACGTTCATTCGCAGAACTTAAGTTTCTGGAGG GCCTATAAATCTGCAGAAGTGAGAGCAGATAAGAAGCCAGAAATTGCTTTGTAAGCAAACATCAGAGCTTTAA ATTTGAGCAACAACTGGCAGCCAGTGCAAACGGATAAGTAGCGGAGTGACATGTGCTCTTTTAGGTTCATTAA AGACCACTTGTGCTGCTGCGTTCTGAAGCATCTGAAGAGGTTTGATAGAGCTTGCTGGGAGCCCGGCTAGTAG AGAGTTGCAATAGTCCAGTCTGGAGAGAACAAGAGCTTGAACAAGGAGTTGAGCTGCATGTTCAGATAGGAAG GGTCGGATCTTTCTGATGTTGTAGAGTGCGAATCTGCATCGATCGAGCAGTTCTAGAGATGTGGTCAGAGAAG TTTAGCTGGTCATCAATCGTTACTACAAGGCTTTTTCCCATTTGTCCCATCCATCTGGATTGAAAAGTTATGA TGTAGAGTCGGGTTGGCAGAAACAGGTCTCCATTTACACACAAAAAATAGGCAATAACCTATGAAAATTAAAT AATAATAATAATAGAAAACATAATGAAAATGTCTACGAGACAAATCCAGAAAAAGTAGAAATAATTATTTAAC CATGTCAACTAAACAATACTTGCACCATGTGTAAGACTTTTACATAACAAGACAAGACCACAGAGGGGTCAGT CGAGTGGACATTGGACTGTGCTCAGTGAAGGGAAGTGGTTTGGCTTCTGTCCCTGTGTGTTGTCTCAAACATT GCTGTTATTCTCTGTTACTTGGCTCTCCCCATGAGAAAATGAACAAACCTCCACCCACAAGTCATACAGAATT AGACATAACCATCCCAGAGATCAGCACACATTGTGTGTGATGAGCTGCCATCAGTAATTAAGTTTAGGAACAA TAAAAAAAAAATAAAAAAAAACATTATTGAAATGTTTGTCATAATTATGAAAAATTAAACAGAATATTTGTAC TTCTTTTTTAACTATTATTTTTCACAAAATGCATTTGCTGTGAACACAACGTAAACCTTTCTTACAAAGGAAG GACCCACCGGCAGCATAATATACAGTAAGTGCGCTATAAATTTAATATGTCTGACAGTACCCACAATTCCCTT CCTTGTGGCTTTCTGCCCACAGTTTGCTACAAGGGACAAACAACTTATTTGACGAATTATGAAACTCTAGGAC CACAAAGGCCGTATTGATGTGACGACATATTTAAGTGACGCAGTTTCCAGTGTGCAGAGGAACACTTTGTTCT GCGATGAATTAAAGATGCCTTGTTTTTTTCTAAGAAAGACATTCCAGCAACAAATATGTCATGTGTGATGTTG AGAAGTTTATATCAGGACCACCCGGAGATTCTGACATTCAAGGAAATTCCCTTTCCACCTCCAGTCTTTACTA TAACTTATAGTACAAAAAAATTAAAATAATAACAATCTACTTTTAGTTGAAAAGAGACATGAACTGGCATTCA AATGTCACAGACACCTGAGGCATATTTGAAGGTCATCCAGTACCATAACAAAAAATAGTAACACATGTATTTG TAATGCAAAACATTTTGCCTTTTAAGGAGATATAATGCTGCGTATCATCTGTATAATCATAAAAATGAATTCC CTGCTTCCTTATGACATTCCAGAATTACAAAGCGCCTAAAACAGCGCCTTGTGGCACACCTTGATCACAATTT GCTCTTTACAAACAGACAAGTAGGCTTTTGGTAGCCCAATATTGTTTTTTTTAATACAAAATAAATATCTAGA TCTAAATAAAACATCTAACTAACTACCCAGAGGTTTAACCATAAAGTCACTATGAAATACTGACTTCTACAAA TCTGCATTGTAATTCCTCCACATCATAATTATGAATTTAAATAAAAGGAAAATGAGGAAAGAAAACTCCTAAA GGGAACCTATTTTATGCATTTTACAGTACGCAAGATAAGCCTTTGGTGTCTCTAGACTGTGTCTGTAAAGTTT CAGCTCTAAATACACATCATCTTATCACAATGCTGGAGAAATTGTGAAGTAGGGGACTATGTTCACATATTTT AGGAATGTCCCAAAATACAAGCGTATTGTTAAAAGGGAGATTAGTAAAGTATTAGGATACGACATATCAATGC ACACAGTATTTTATTTTTATTTTTTGCTTGATGGCTTCCCCTCTGAGCAGTTCAGCAAGTCTCACCTATTTGC ACTATATGTATTATTAATAACAGCTAGGAAGATAATTCCAGTGAAGTGGGTGAAGACCAGCTGACCTACTATG ACAGAATGGACACAGACGCTGGAACAGATATACAGTTGTCAAAATTATTAGCCCCCCTGTTTATTTTTTCCCC TAATTTCTGTTTATCAGAGAGAAGATTTTTTTTAAAATACATTTCTAAACATAATAGTGTAATAACTCATtTC 
TAATAACTGATTTATTTTATCTTTGCCGTGATGACAGTAAATTATATTTTACTAGATATTTTTCAAGACACTT TTATACAGTTTAAAGTAACATTTAAAGGCTTAAGTCAGTTGATTAGGTTAACTAGGCGGGTTAGGGAAACTAG GCAAGTTATTGTACAACAATGGTTTGTTCTGTAGACTATCAAACAAATATAGCTTAAAGGGGCTAATAATTCT GACCTTAAAATGGATTAAAAAAAAACTGCTTTTATTCTAGCCGAAATAAAACAAATAAGACTTTCTCCAGAAG AAAAGAAAATAATTAAAAAAGAAAAAAAAATCAAAGGGGGGCTATTCATTCTGACTTCAACTGTATATATTTA AAAACATGACTACAGATTTGCAGTCAAAATATTTTCCAACAGACCTGGGCATTAATCAAAACATACTGACTGG ATCCTTGAAATGCGTTCTCAGGGGCTTTGTTGTAGTTATTGCCTTTATATTGAAGTTTATTTATCATTATTTT ATATTATCAGTGATGTGTTCTGTATGAGATATCAAGGTTGTAAAATGAAGGTACCATACACACTCACCTCTTG GCGCACGTAATGCTGCATTCATCTGCCCTGACTGTGCCCAAAGATGTCAGACTATTTCCATAAATTCTTTGGT GAAGAAAGTAGGCGTTTAGTGAATTCACAATGTCTCTGAATCTGATAGAATCACACAAAGAGGGCAAACTGAA GGCACAAATTAGCTTTTGTGCTCTCTGTAACAATTTGTTTTTGTTTTAAATTTCAAATTCCTAAATAATCTTG ATGCTTTAAGACAGAATGCATAAAAAAAGAGGGGGGACAAATGATGACAAGATACATGTGGTACATCGGGTAA TATTTTACTTTGTGTCCACGATGAAGTATATAAATATGTATTTTTTTATTATAATGCATTATAGTGGTAAATG TGTCTGAACTGCCTATAATGTATTTATTTATTTATTTATTTATTTATTTTAAGAAATAAACGTAATAATGGAT

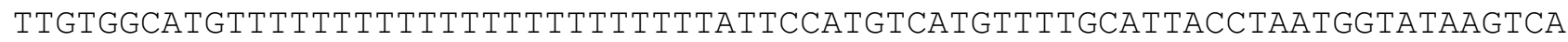
GGTAAGTAAAATAAGTAGGCAAAATTTATTTGGTCACACTTTATTTTATTTTATTTTAATTCTCGAAATTTTC AAAGCATAAACCAAGACCTTTAGCTCAACAAAGTACTAATTTGCTACTGTTTGCTAATTTCTATCAAACTACT AAACACTAAAAAAATGAATTTTGCTGCTTGTTCAAACTACCTATTTAAAATGGGTTAAAACAACACAATTACT TTGTGTTTTTTTATTTCATTTAAACTTAATGGTAATATGTTAAATCCACTTAAATATGTAAAAACGATTAAAT TAATCGATTTGTGTTGGGACAAAATAAAGTAATTGTGTGAAACGCGTTTTTTTTTTACTGTGTAGTTGTGTTA AGGTATTGGGTGTAGGATTAGGGATGTAGAATTAGATACTTTATAATTACTAATAAGTGGCCAATACCTTAAT AATAGGCAGTTAATAAGCCAGTAGGTAGCAGTGGAAATTGGTACATAAACCAAAGTGTTACCATTCGTTTATA CTGTATAGTACATCTAACATTAAGTGATATACAATTTACACACTATTTCACATAAATATATCTGTGACATGGC ATAATGAGGGAGCTACTCAATGAACTGTTGAAAATATTGAAAATATTTAAATTCTGTTTCACAAAAAGTTACA CAAAATATTAAATCACACACTTTACATCCATTTAAAATCACTTTTGTAAATGTATTTTAAAGCAGACTCCAAA ATGGAGGCTTGACTTTGATCAATAAACATCCATTTTAGAAAAACAAAACTTAAAGATGCCTTTTACAGTACAG GATTGTCAGTGACGAAGCAGATCCACGGTTGAAGGCAGAATAATTAGCCCTTGTTAATTATTATCCGCCCTGT GTATTTCTGTTTAACGGAGAGAAGATTTTTTTCAACACATTTCTTAAGATAATAGTTTTAAGAACTAATTTCT AATAACAGATTtTCCTTATCTTTGCCATTATGACAGTACATAATATTTTAGATATtTTtCAAGATACTAGTA TTTAGCTTAAAGTGTGATTTAAAGGCCTAACTAGTTAGGTTAACTTGGTGAGTTCGTTGTATAACAATGGTTT CAAATTTTTTTTGCTTATATATAATGTAGGTCACACTTTACAATAAGGTTCATTAGTTAATGCTAAGTAATGC ATTTACTAACATGAACAAACATTGAACAATACATTTACTACAGTATTTATTAATGTTAATAAACGTTAGTTAA TGAAAATAGAATAATAGTGACCTTAAAATGGTTTAAAAACTAGCCAAAATAAAACAGATAAGACTTTCTTCAG AAGAAAAAAACATTACAGGAAATGTGAACATTTCAACAGAGGATTACTGTGAAAACATATAGCCGTATACATT TCTGAAGAGCATGAATTATGTAGCCAGAGCTACGTATGGCTGCATTTCCTCTTTAAAACGAACAATCCAGGGT GGTATGATGCTGACGATAGATTTTGTTTTTTTTTACTCTTCCAGCTGACTGCTTACCTACGTGTGGGCGGCTT TTCTGCTGTTACCAGTTTGCCCAGTAGCTTGCCATGTCCATCGGTGGACTTTAGACAAAGAGAGGAGTTGACT GTGACGACAGGGTTTGAGTCTGGTAAAGAATGGTTCCAGAAAGCAGCTAAAACCAAAACAAAAGGCAAAATTT ATTTAGACAAGTACAGTTAAAACCGGAAGTTTACATACACTGCATAAAAAGGCACATAACCACTTAAAAAAAG TCAGATGTTAATGTAAACTTTTTCTCTTTTAGGTAAGTCAGGGTAATCAAATTTGTTTCTGTTATGCTTAATA TCAGAATAATGAGGGAGATATTTTTGAGAAATTGTTATAACTTTTCTTGAAAGTCAAGTTTACATACAATAAG ATTATTATGCCTCTGAAAAAGCTCAGATGATGTTGTTACAACAGTAGAATAGTATTAAAGGAAAACCTCAACC ACACAGCTTCCTTGTGTGGCAACATGGGGAAATCTACAAGCCAGAATCAACAAAAAAGCCAGATTAAAATTAG CTAAATTGCACAGGGAAAAAGACACATTTTTGGAGACATGTCCTGTGGTCTGATGGAACTAAGATTGAACTGG TGGGCCATAATGATCTGTGTTACATTTGGAGGACAAAGGGGAAAGCTTACAAGCCTAGGAACACCATCCCAAC TGTGAAGTATGAGGGCAGCAGCATCATCTTGTGAGGCTGTTTTACTGCGGGAGGGACTGGTCCACTTCACAGC ATAGATGGCATCATGGACAAAGAACATTATGTTGAAATACTGAGTCAACATATCAAGACATCAGCCAGGAAAT TACAACTTGGCCACAAATGGGTCTTTCAAACAGACCATGACCCTAAGCATACTGCCAAATTAGTTCAAATATG CTTTAAGGACAACAGAGTGAATGTTTTAGAGTGGCCATCACAAAGCCCTGATCTCAATCCTATAGAAATTTGT AGGCAGCTTCTGTGAGCAAGACAGCCAACAAATCTGACTCAATTACACAGTTCTGTCAGGGGGAATGGGCCAA AATTCCTTTTAGCTATTGTGAGAAGCTTGTGGAAGGACACCCAAAACATTTGACCAAAGTTAAACAGTTTAAA AGCAAAGCTAAAAAAATACATCTAACAAGGACATGTATGTAAACTTTTGACTGTCTAAAAAATTCTATCATTA TTCTGGCATTTAGCAAATGTAATTAATTTAGGTAATCTTAACAGACCCAAAATAGTAAAGATTTAGTATGATT TACCATCAGAAATTTCTTTAAAAAAAAGTTATGTTCCTTTTTTAAAGTGTATGAAAATTTGTGGATTCAACTG TAAATAACAGGGTGAAAGTGTGGTAAAAACTAAAACTGTAGTAAAAATGAGGCAAGGCCTTTTTTGGATTGCT 
TTCGAAAACACTGTTGCTAAGGTTTAGGGAAGGGGTTAATCGGATCAATTGGTGCTTTTGAAAACACTATTGG TCGGGTTTAGGGTAAGGAATGGGGGGGGGGGTCAATTAGTCATTCAGTCAGTCAGTCGGTCAGTCGACAGCAG CCTCAGGTGGATTTATGCCAGAATAGTAGGTATGTATGTCACTCATTTGGGAGATCAGAAAAGGCACACACAG CAGTGGATTCTCGAAAACAAAAACTGCAAAAACTTGTTTCTCCTAGGATGTATTATGCAATATCCAGAAATGT ATATAAAGGGTACATATCAATAATGAGCCTGGATTGGAAAATAAATCCTTGCTCTGTTAAACATCATTTAAGA AATATTTGAAAAAGGGAAAAAAATTAAATAAAATCACAGGAGGGCTAATCATTTTGACTTCAACTGGATATAA TGTGAGCAGCTTGTGAATGAATCTGCATTGCATTTACTGTAAAGCTTAACAGTCAAATGATAAATGAACATGA TAAATGAAGGTGCATCAAAATAAGGACACTTTTTGTTTGTTTGTAGGCCTGATGTTTTCTCATTTGACTGCTG TAGAATAACTCGAGGACGTGCCGCAGATGTCTAATATCCTTTCAAGTCTTTGAAGTCCTGATGAAAACCCAGA GATGACTTGCACACAGCTGAGGGTTTGAGTGTCATGGTAAATGTTTTGGAAACTCGACGCTGAAGGACACAAA GGAGCACACAAGGTTACGCAGCGTTTTTATTTTGACTTTTGAATTATCCTGATTGAGTCCCATGCAGCTGCTG GGATATAAAAGTGTACGTCTGCAGTTCAAAGGCTTTAGACTTGCTTCTTAATCCGTTCTTATGGCAGGACCCA ACTTTTGTATACAAAGTGGT 
Supplemental figure 5: Specificity controls of anti-GFP and anti-Cyp19a1a antibodies to zebrafish gonads. GFP (green, A-D) protein was immunolocalized both in testis (Left) and ovary (Right) of mature zebrafish. A: testis of a cyp19a1a-GFP transgenic zebrafish showing a labeling in Leydig and germ cells. B: ovary of a cyp19a1a-GFP transgenic zebrafish showing labeling in perifollicular cells. C: testis of a wild type zebrafish showing a very slight green fluorescence background. D: ovary of a wild type zebrafish showing no labeling. Cyp19a1a $(\mathrm{E}-\mathrm{H})$ protein was immunolocalized in testis (Left) and ovary (Right) of zebrafish. Cyp19a1a specific labeling is detected in the Leydig and germ cells of the testis (E) and in the peri-follicular cells of the oocytes (F). When this anti-Cyp19a1a antibody is preincubated with the synthetic peptide $(100 \mu \mathrm{g} / \mathrm{ml})$, no labelling is observed in the testis $(\mathrm{G})$ and only a slight unspecific labeling is observed in ooplasm of oocytes $(\mathrm{H})$. Without any primary antibody (secondary antibody: AlexaFluor ${ }^{\circledR} 594$ ), no labeling was observed neither in the testis (I) nor in the ovary (J). Nuclei are in blue. Imaging was realized with a Zeiss Axiolmager Z1 fluorescence microscope equipped with an MRm5 camera combined with an ApoTome. Acquisition parameters were the same for all GFP photomicrographs and for all Cyp19a1a photomicrographs. Scale bars: 20 $\mu \mathrm{m}$. 


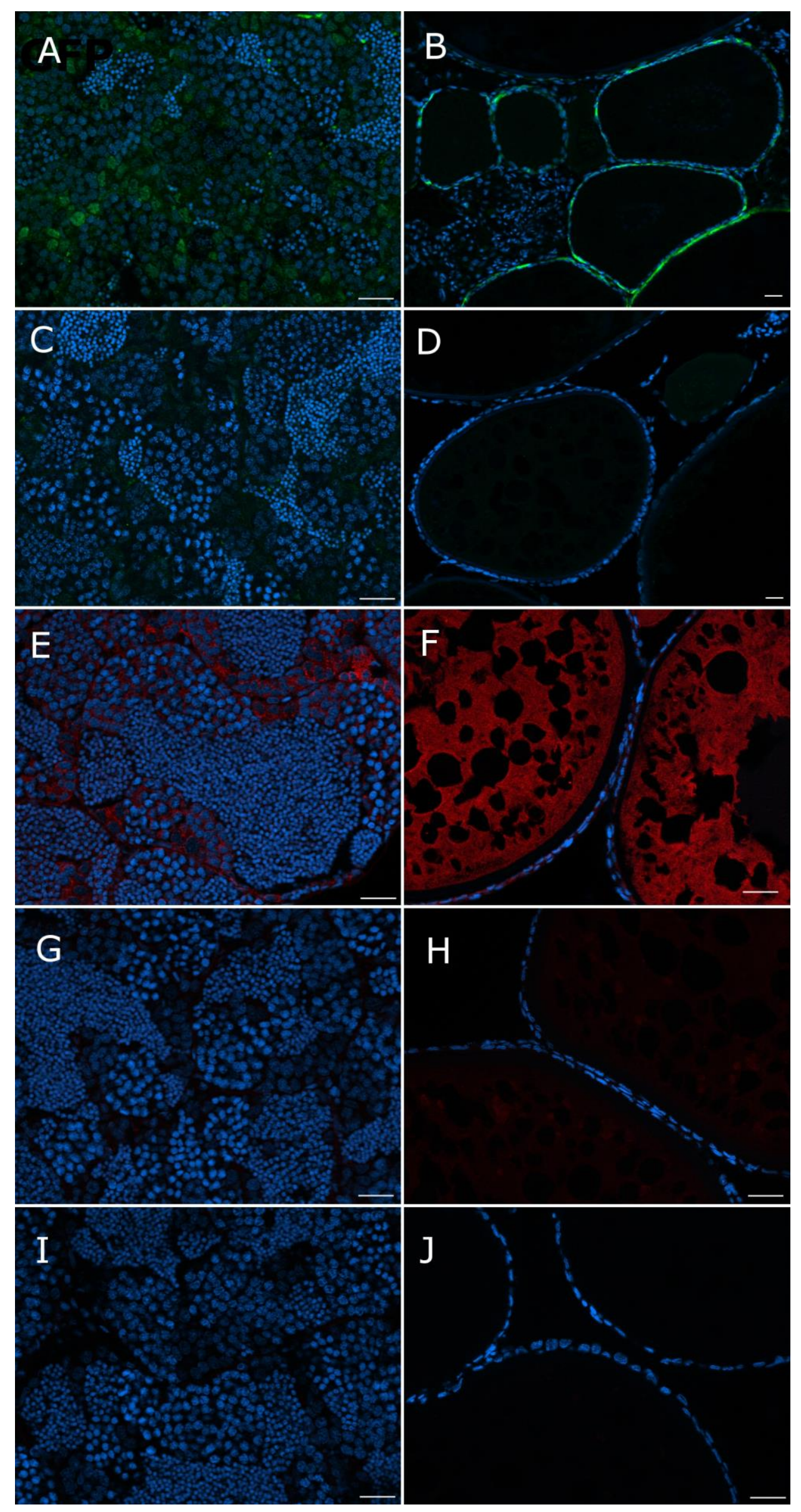

